\title{
Resolving Over-Constrained Temporal Problems with Uncertainty through Conflict-Directed Relaxation
}

\section{Peng Yu \\ Brian Williams \\ Cheng Fang}

Computer Science and Artificial Intelligence Laboratory, MIT

32 Vassar Street, Cambridge, Massachusetts 02139, USA

\author{
YUPENG@MIT.EDU \\ WILLIAMS@MIT.EDU \\ CFANG@MIT.EDU
}

CUI.JING@ANU.EDU.AU

PATRIK.HASLUM@ANU.EDU.AU

\author{
Jing Cui \\ Patrik Haslum \\ Research School of Computer Science, ANU $\&$ \\ Decisions Sciences Program, Data61 \\ Canberra, Australia
}

\begin{abstract}
Over-subscription, that is, being assigned too many things to do, is commonly encountered in temporal scheduling problems. As human beings, we often want to do more than we can actually do, and underestimate how long it takes to perform each task. Decision makers can benefit from aids that identify when these failure situations are likely, the root causes of these failures, and resolutions to these failures.

In this paper, we present a decision assistant that helps users resolve over-subscribed temporal problems. The system works like an experienced advisor that can quickly identify the cause of failure underlying temporal problems and compute resolutions. The core of the decision assistant is the Best-first Conflict-Directed Relaxation (BCDR) algorithm, which can detect conflicting sets of constraints within temporal problems, and computes continuous relaxations for them that weaken constraints to the minimum extent, instead of removing them completely. BCDR is an extension to the Conflict-Directed A* algorithm, first developed in the model-based reasoning community to compute most likely system diagnoses or reconfigurations. It generalizes the discrete conflicts and relaxations, to hybrid conflicts and relaxations, which denote minimal inconsistencies and minimal relaxations to both discrete and continuous relaxable constraints. In addition, BCDR is capable of handling temporal uncertainty, expressed as either set-bounded or probabilistic durations, and can compute preferred trade-offs between the risk of violating a schedule requirement, versus the loss of utility by weakening those requirements.

BCDR has been applied to several decision support applications in different domains, including deep-sea exploration, urban travel planning and transit system management. It has demonstrated its effectiveness in helping users resolve over-subscribed scheduling problems and evaluate the robustness of existing solutions. In our benchmark experiments, BCDR has also demonstrated its efficiency on solving large-scale scheduling problems in the aforementioned domains. Thanks to its conflict-driven approach for computing relaxations, BCDR achieves one to two orders of magnitude improvements on runtime performance when compared to state-of-the-art numerical solvers.
\end{abstract}




\section{Introduction}

Every day, as individuals we miss meetings and deadlines, because we try to do too much, and do not estimate time accurately. These situations can lead to anywhere from a minor annoyance, such as being late for lunch, to a major catastrophe, such as missing a flight. Due to the scale and complexity of such over-constrained problems, it is often impossible for humans to find good resolutions independently: we perform poorly in explaining the cause of failures, assessing the source of uncertainty, and making trade-offs between a large number of constraints. These situations can be better handled with decision aids that help individuals estimate how much time is required in order to compensate for uncertainty, and by providing advice on which requirements should be dropped, to achieve a manageable set of goals.

Such over-subscribed situations can be modeled by inconsistent temporal problems. A temporal problem is inconsistent if no schedule (Dechter, Meiri, \& Pearl, 1991), or execution strategy (Vidal \& Fargier, 1999) for problems with uncertain durations, can be found that satisfies all its constraints. For chance-constrained probabilistic temporal problems (Fang, Yu, \& Williams, 2014), inconsistency means that no strategy for executing its activities exists such that the chance of violating any temporal constraints is lower than the threshold of the chance constraint. To repair an over-constrained temporal problem, one can identify its conflicting constraints, similar to past work on diagnosis, and resolve the conflicts by relaxing one or more of them such that the feasibility of the problem is restored. In addition, since acceptable risk levels may be negotiable in some situations, we can restore the feasibility of chance-constrained problems by identifying constraints that cause the probability of failure to exceed the chance constraint, and increasing the level of accepted risk accordingly.

Several methods have been developed to solve over-constrained temporal problems. Beaumont, Sattar, Maher, and Thornton (2001) applied partial constraint satisfaction techniques to find a subset of satisfiable constraints. Later, disjunctive constraints and optimality were added in the context of over-constrained Disjunctive Temporal Problems with Preferences (DTPPs) (Peintner, Moffitt, \& Pollack, 2005). In a DTPP, the disjuncts of every constraint are assigned a preference function that maps the temporal constraint to a cost value. The optimal partial solution is obtained by enumerating consistent subproblems using Branch \& Bound, as well as other optimization techniques introduced by Khatib, Morris, Morris, and Rossi (2001). Most of the prior work has focused on restoring consistency through complete suspension of constraints, with the exception of Lanz, Posenato, Combi, and Reichert (2015), which restored controllability by tightening uncertain durations. However, in real-world scenarios, the user often wants to preserve as much of the schedule as possible to minimize the perturbation. In addition, most prior work focused on temporal problems with only controllable durations. When applied to many real-world scenarios with uncertainty, their relaxations may fail since they only satisfy a subset of the possible times for the uncertain durations.

In contrast to the work on discrete relaxation, in this paper we focus on continuous relaxation, in which bounds on timing constraints are relaxed at some level of cost. Our approach, the Best-first Conflict-Directed Relaxation algorithm (BCDR), leverages prior work on hardware diagnosis (de Kleer \& Williams, 1987; Williams \& Ragno, 2002): instead 
of mode assignments, it diagnoses conflicts between temporal constraints, and computes continuous constraint relaxations to resolve these conflicts and guide the search away from infeasible regions. BCDR is able to generate the most preferred relaxation faster than other state-of-the-art algorithms, and enumerates multiple preferred relaxations in best first order, rather than just the most preferred relaxation. In addition, for chance-constrained temporal problem, BCDR also learns the source of risk through grounding probabilistic temporal constraints to set-bounded uncertain duration using risk allocation, and applying controllability checking algorithms to identify conflicting constraints from the grounded problem. Resolutions to these conflicts can then guide us to find feasible relaxations for temporal constraints and/or relaxations for the chance constraint. Depending on the problems given, BCDR can be configured to work with Simple Temporal Constraints (Dechter et al., 1991), Set-bounded Uncontrollable Temporal Constraints (Vidal \& Fargier, 1999), and Probabilistic Simple Temporal Constraints (Tsamardinos, 2002). For the second and third types of problems, the users can choose to relax and enable either a static schedule, or a dynamic execution policy for events in these problems.

A preliminary version of several sections in this article appeared in the proceedings of prior International Joint Conference on Artificial Intelligence (Yu \& Williams, 2013), International Conference on Automated Planning and Scheduling (Yu, Fang, \& Williams, 2014; Cui, Yu, Fang, Haslum, \& Williams, 2015) and AAAI Conference on Artificial Intelligence (Fang et al., 2014; Yu, Fang, \& Williams, 2015). This paper unifies and extends their presentation as follows: (1) a unified definition for temporal relaxation problems involving a) conditional temporal constraints, b) set-bounded uncertain duration and c) probabilistic uncertain duration with chance constraints; (2) a new conflict resolution strategy that improves run time performance in some scenarios by nearly $100 \%$ compared to the previous one used by BCDR; (3) additional empirical study results, test cases, and configurations that demonstrate the application of BCDR in different domains.

This article is organized as follows. In Section 2 we present the definition of the temporal relaxation problem and its variations. We illustrate the inputs and outputs of the BCDR algorithm using examples from the domain of urban travel planning and deep-sea exploration. Section 3 introduces the approaches we developed to resolve over-constrained temporal problems with three different types of constraints: conditional temporal constraints, set-bounded uncertain duration and probabilistic uncertain duration with chance constraints. We discuss the applications and empirical evaluation of BCDR in Section 4. Finally, Section 5 concludes with recommendations for applications and future improvements.

\section{Problem Statement}

In this section, we present three definitions of the temporal relaxation problems, which differ in their treatment of uncertainty. The development of BCDR is motivated by a set of real world scheduling problems, especially in the domain of deep-sea exploration. We have been collaborating with ocean scientists at the Woods Hole Oceanographic Institute to develop planning systems for managing their vehicles for deep-sea explorations. In their expeditions, the unbounded uncertainty in the environment, the underwater vehicles, and the crew performance make it impossible to find a plan that offers a $100 \%$ guarantee of success. 
Moreover, their expeditions are often overloaded with scientific experiments, equipment maintenance, and unexpected vehicle failures, which require adjustments of their goals and plans frequently. Therefore, correct handling of uncertainties and the trade-offs between mission requirements are essential for the success of their expedition. BCDR was developed with the objective to help them address these issues and alleviate their work load. We use a rich problem model to cover all types of uncertain durations, temporal constraints and decisions they have to deal with. We start our presentation with the simplest temporal problem formulation with only simple temporal constraints, then discuss the extensions to the formulation for supporting uncontrollable durations and chance-constraints. As described in Section 1, BCDR is capable of handling temporal problems with the following three types of constraints:

- Simple temporal constraints (Dechter et al., 1991) where the duration between lower and upper bounds are controllable. This type is often used for modeling requirements between temporal events. For example, to get home in 40 minutes, given that the subway takes 35 minutes, we know for sure that the requirement can be met.

- Set-bounded uncertain durations, also called contingent constraints (Vidal \& Fargier, 1999), involving uncontrolled durations, represented by interval bounds. Compared to simple temporal constraint, it models the temporal duration as a random variable. The modeler makes a commitment to a degree of robustness by specifying the interval of outcomes to be handled. For example, to get home in 40 minutes, given that the subway takes anytime between 30 and 45 minutes. Unlike the previous example, there is no guarantee that the requirement can be met due to the uncertainty in the subway time: there is a chance that the ride may take more than 40 minutes.

- Probabilistic temporal durations (Tsamardinos, 2002) with information on the likelihood of outcomes. This type is more complex compared to the other two since the uncertainty in duration is accurately modeled using a probability distribution instead of a pair of temporal bounds. In addition, it also allows explicit representation of requirements on risk taken through a chance constraint. For example, we can specify a $95 \%$ guarantee that you'll be home in an hour, given that the subway takes a mean time of 30 minutes, with a standard deviation of $5 \mathrm{~min}$.

BCDR is designed to be a unified algorithm that solves temporal problems with all three types of constraints, and we will discuss how these problem definitions build on each other. Throughout this section, we will be using an example from the domain of deep-sea exploration on scheduling activities for autonomous underwater vehicles to survey the sea

floor. We use the example to illustrate the inputs and outputs of BCDR, as well as how it collaborates with the ocean scientists to find resolutions for the over-constrained problems.

\subsection{Controllable Conditional Temporal Problems}

Consider the following example in which an ocean scientist, Rich, is planning to deploy an autonomous underwater vehicle (AUV), Sentry (Figure 1a), to survey the sea floor close to the coast of Northern California. This mission is expected to start at 11:00AM from the R/V Atlantis (Figure 1b), and Rich has reserved the vehicle until 2:00PM. During this 
mission, he would like to visit one of the two asphalt mounds (Figure 1c). The two sites are denoted by Location A and B. Rich has a preference over the two options and their required survey times vary from 35 minutes to 50 minutes. After visiting the mound, Rich wants to scan one of the three nearby methane seeps (Figure 1d), denoted by Location X, $\mathrm{Y}$ or Z. It takes a different amount of time at each site due to their sizes and complexity. Finally, traversal times to and between these sites are different, and the robot must return to the ship in three hours (11:00AM to $2: 00 \mathrm{PM})$ so that the next scientist can start their mission on time.

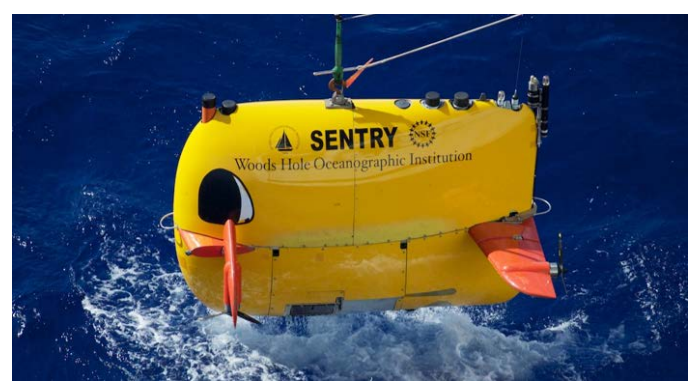

(a) Autonomous Underwater Vehicle, Sentry

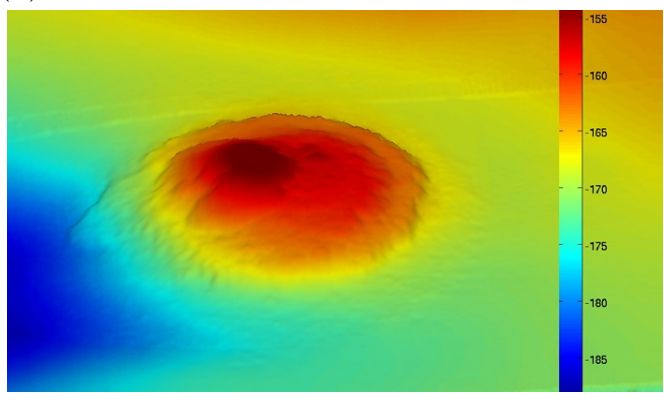

(c) Sonar data of an undersea asphalt mound collected by Sentry

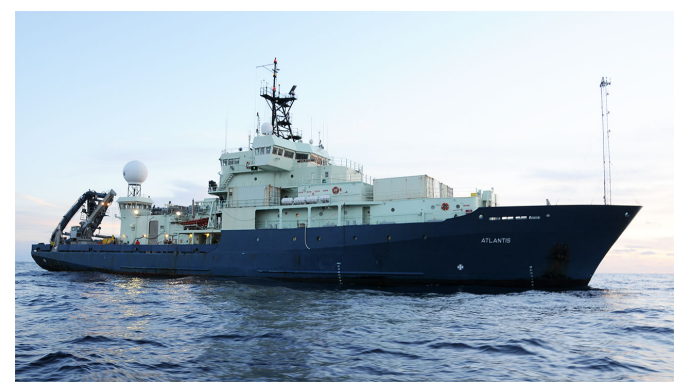

(b) Research Vessel, Atlantis

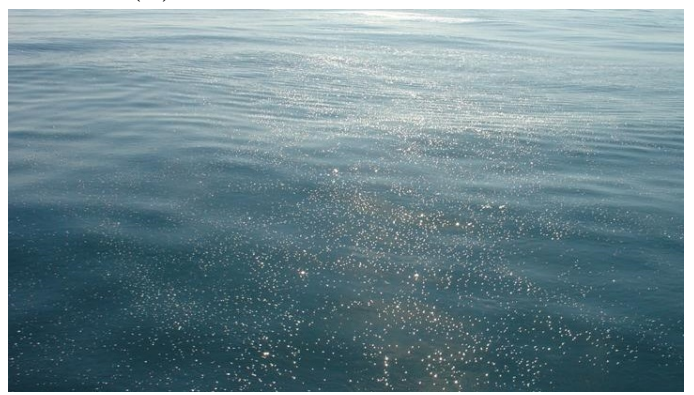

(d) An active methane seep in South Ellwood Oil Field (Photo by R K Nelson)

Figure 1: Vehicles and survey targets of the example expedition (Courtesy WHOI)

We define the Controllable Conditional Temporal Problem (CCTP) formalism for modeling Sentry's mission and the best plan for Rich, which includes: which asphalt mounds site to survey, which methane seep site to scan, how much time to spend at each site, and whether to extend the mission length. We start by defining two variables for the choices he needs to make: AM (asphalt mounds sites) and MS (methane seep sites). AM has two options in its domain: A (40) and B (100). Each option is associated with a positive reward value that represents Rich's preference towards it, the larger the better. The other variable MS for methane seep sites has three options: X (73), Y (80), and Z (47).

Next, we define twelve time points in the problem (Table 1): a reference point in time $(S)$ that represents the beginning of the trip at 11am; a time point that indicates the end of the trip $(E)$; and time points representing the arrival and departure of each location (asphalt mounds sites A and B, methane seep sites X, Y, and Z).

Table 2 shows all the conditional constraints in the CCTP that encode the temporal relations between events. A subset of these constraints only hold for some choices, such as 


\begin{tabular}{|lc|cc|}
\hline \multicolumn{4}{|c|}{ Time Points } \\
\hline Mission starts $\quad S$ & asphalt mounds site A arrive/leave & $A_{A}, A_{L}$ \\
Mission ends $\quad E$ & asphalt mounds site B arrive/leave & $B_{A}, B_{L}$ \\
\hline methane seep site X arrive/leave & $X_{A}, X_{L}$ & \\
methane seep site Y arrive/leave & $Y_{A}, Y_{L}$ & \\
methane seep site Z arrive/leave & $Z_{A}, Z_{L}$ & \\
\hline
\end{tabular}

Table 1: Events in Sentry's mission CCTP

\begin{tabular}{|lll|}
\hline \multicolumn{3}{|c|}{ Constraints (in minutes) } \\
\hline$C_{1}(\mathrm{R}): A_{L}-A_{A} \in[50,60]$ & $C_{6}: A_{A^{-}} S \in[45,65]$ & $A M \leftarrow A$ \\
$C_{2}(\mathrm{R}): B_{L^{-}} B_{A} \in[45,60]$ & $C_{7}: B_{A^{-}} S \in[30,50]$ & $A M \leftarrow B$ \\
$C_{3}(\mathrm{R}): X_{L}-X_{A} \geq 60$ & $C_{8}: E-X_{L} \in[28,35]$ & $M S \leftarrow X$ \\
$C_{4}(\mathrm{R}): Y_{L^{-}} Y_{A} \geq 65$ & $C_{9}: E-Y_{L} \in[30,32]$ & $M S \leftarrow Y$ \\
$C_{5}(\mathrm{R}): Z_{L}-Z_{A} \geq 100$ & $C_{10}: E-Z_{L} \in[50,60]$ & $M S \leftarrow Z$ \\
\hline$C_{11}: X_{A^{-}} A_{L} \in[51,54]$ & $A M \leftarrow A$ and $M S \leftarrow X$ \\
$C_{12}: Y_{A}-A_{L} \in[42,45]$ & $A M \leftarrow A$ and $M S \leftarrow Y$ \\
$C_{13}: Z_{A}-A_{L} \in[30,55]$ & $A M \leftarrow A$ and $M S \leftarrow Z$ \\
$C_{14}: X_{A}-B_{L} \in[22,24]$ & $A M \leftarrow B$ and $M S \leftarrow X$ \\
$C_{15}: Y_{A}-B_{L} \in[21,25]$ & $A M \leftarrow B$ and $M S \leftarrow Y$ \\
$C_{16}: Z_{A}-B_{L} \in[30,35]$ & $A M \leftarrow B$ and $M S \leftarrow Z$ \\
\hline$C_{17}(\mathrm{R}): E-S \in[0,180]$ & \\
\hline
\end{tabular}

Table 2: Conditional temporal constraints in the CCTP of Sentry's mission

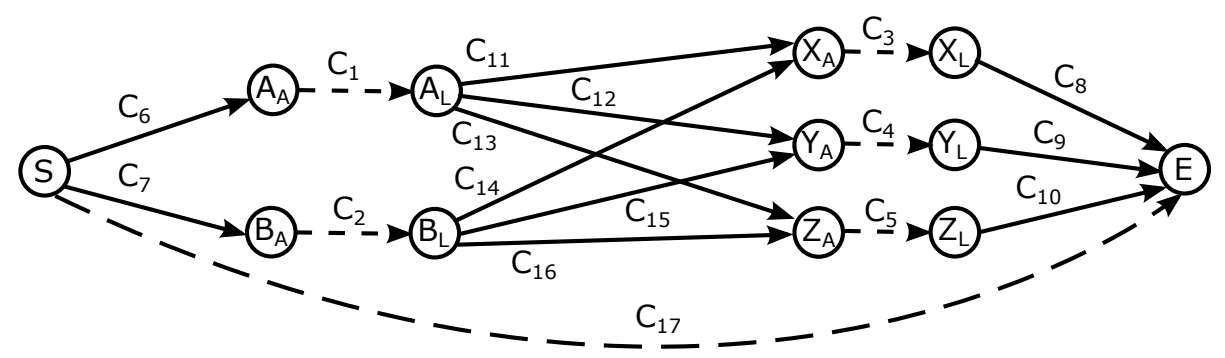

Figure 2: A graphical representation of Rich's mission CCTP

the duration of survey at site $\mathrm{A}\left(C_{1}\right)$ for assignment $A M \leftarrow A$. We represent this by saying that constraint $C_{1}$ is $A C T I V E$ only when the choice $A M \leftarrow A$ is made, and indicate this by labeling the constraint with $A M \leftarrow A$. The label is called the guard of the conditional constraint. In this problem, constraints $C_{1}$ through $C_{5}$ represent Rich's desired length of survey at five locations. For example, $B_{L}-B_{A} \in[45,60]$ indicates that Rich would like Sentry to spend between 45 and 60 minutes at asphalt mound site B. These constraints are labeled by the assignments made to the decision variables: a constraint is activated only if its label assignment is made. For example, $C_{2}$ will be considered only if Rich chooses to visit site $B$, as shown in the right side of Table 2 . Constraints $C_{6}$ through $C_{16}$ are simple temporal constraints that encode the traversal time required between locations. They are 
conditioned on assignments made to either $A M$ or $M S$, or both $\left(C_{11}\right.$ through $\left.C_{16}\right)$. Finally, $C_{17}$ constrains the duration of Sentry's mission to three hours. Similar to previous temporal network representations in literature, the CCTP can be visualized using a directed graph (Figure 2), in which nodes represent events and arcs represent temporal constraints.

Some of the constraints followed by a symbol ' $\mathrm{R}$ ' (also highlighted in dotted arcs in the graph: $C_{1}$ through $C_{5}$ and $C_{17}$ ) are relaxable temporal constraints. Their lower and/or upper bounds can be relaxed in order to restore the consistency of the problem, if necessary. Each relaxable constraint comes with one or two cost functions that describe Rich's preferences towards the weakening for their upper and lower bounds. These functions map the relaxation from $L B$ to $L B^{\prime}$, or from $U B$ to $U B^{\prime}$, to a positive cost value, as seen in Figure 3. If the upper bound of $C_{17}$ is increased from 180 minutes to 200 minutes, meaning that Rich extends his mission by 20 minutes, the cost will be 40 . On the other hand, if he shortens the survey time by reducing the lower bound of $C_{3}$ to 40 , the cost would be 80 . In this example, we assume that all other relaxable constraints have linear cost functions with gradient 1 for simplicity, but the approach is generalizable to arbitrary monotonic functions (decreasing for lower bounds, and increasing for upper bounds).
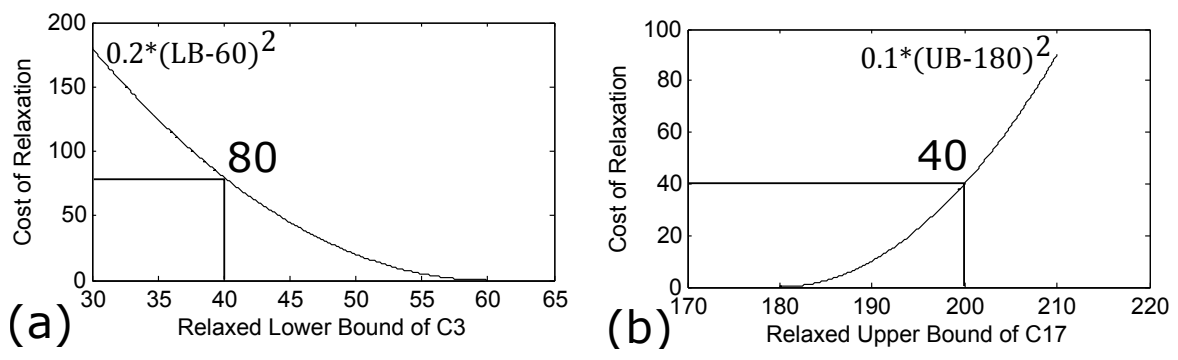

Figure 3: Preference functions for $C_{3}$ and $C_{17}$

\begin{tabular}{|l|l|l|l|l|}
\hline Relaxation 1 & & Relaxation 2 & & Relaxation 3 \\
\hline$A M \leftarrow B$ & Rich told & $A M \leftarrow B$ & Rich told & $A M \leftarrow B$ \\
$M S \leftarrow Y$ & system & $C_{3}$ to $\geq 57.5$ & system & $C_{4}$ to $\geq 55$ \\
$C_{2}$ to $\in[39,60]$ & "Do not & $C_{2}$ to $\in[42.5,60]$ & " $C_{2}$ is at & $C_{2}$ to $\in[44,60]$ \\
$C_{17}$ to $\in[0,185]$ & reast 44" & Utility: 169 \\
Utility: 171.5 & relax $C_{17}$ " & Utility: 169.25 & \\
\hline
\end{tabular}

Table 3: Three preferred relaxations to Rich's original CCTP

Before relaxing any constraints, there is no consistent solution to the problem. The cause of failure is that three hours is not enough for Sentry to complete both tasks: traversing to the nearest asphalt mounds and methane seep site will consume at least 80 minutes, which brings the minimum trip duration to nearly 190 minutes. Therefore, one or more temporal constraints need to be relaxed. Table 3 shows three consistent relaxations for the CCTP ranked in best-first order. Relaxation 1 , which is first presented to Rich, suggests visiting site $B$ and $Y$. The survey time for $B$ should be reduced to 39 minutes and the mission should be extended by 5 minutes. The utility of the relaxation is 171.5 , which is computed by summing up the reward of two assignments, $A M \leftarrow B$ and $M S \leftarrow Y$, and subtracting 
the cost of relaxing $C_{2}$ and $C_{17}$. If Rich changes his mind and decides not to relax $C_{17}$, Relaxation 2 will be generated which incorporates this new requirement. It takes Sentry to methane seep site $X$, shortens the scan time to 57.5 minutes and reduces the survey time at $B$ to 42.5 minutes. If Rich is still unsatisfied and adds an additional requirement that survey time at the asphalt mound site should be no less than 44 minutes, Relaxation 3 will be presented, which respects both newly added requirements.

\subsubsection{Definitions}

The example illustrates the modeling of over-constrained temporal problems with CCTPs, and demonstrates the most significant advantage of continuous relaxation: it minimizes perturbation to the original problem. Compared to discrete relaxations, which may ask Rich to give up on the survey for asphalt mound sites or methane seeps, continuous relaxations preserve more of the original problem while restoring consistency. In this subsection, we formally define the Controllable Conditional Temporal Problem formalism, explain its two essential features of relaxable constraints and guarded elements, and present how it is different from other temporal problem models in literature.

Temporal problems with choices can be modeled using Conditional Temporal Problems (CTPs) (Tsamardinos, Vidal, \& Pollack, 2003). We will start by reviewing the definition of this formalism as a stepping stone. CTP is a generalization of the restricted problem class of Simple Temporal Problems (Dechter et al., 1991) by adding uncontrollable outcomes of actions and by conditioning the occurrence of events and simple temporal constraints on the outcomes of these choices. Conditional Temporal Problems (CTPs) are capable of modeling conditional plans and uncertainty during executions.

Definition 1. A CTP is a 6-tuple $\langle P, V, E, Q, L, O V\rangle$ where:

- $P$ is a set of Boolean atomic propositions.

- $V$ is a set of events, each with the domain of the Reals.

- $E$ is a set of simple temporal constraints that restricts the time points in $V$, and are of the form $l_{i j} \leq v_{j}-v_{i} \leq u_{i j}, l_{i j}, u_{i j} \in \mathcal{R}$.

- $Q$ is a set of literals of $P$.

- $L: V \rightarrow 2^{Q}$ is a function that attaches conjunctions of literals, $q_{i} \in Q$, to each event $v_{i} \in V$.

- $O V \subseteq V$ is a set of observation events that provides the truth value for $p_{i} \in P$ through function $O: P \rightarrow O V$.

In a CTP, each event is associated with a conjunction of literals, called a label. If the label of an event is evaluated to be true, the event is said to be activated and needs to be scheduled. Otherwise, the event is said to be inactive and does not need to be scheduled. All temporal constraints associated with an inactive event do not need to be satisfied. 
The solution to a CTP is a dynamic schedule that is able to react to observations in real time, such that all temporal constraints will be satisfied no matter how the uncontrollable outcomes turn out. Conditional Temporal Problems with Preferences (Falda, Rossi, \& Venable, 2010) extend CTPs by allowing fuzzy temporal constraints and fuzzy atomic propositions. It allows the user to specify preferences over the execution time of each event $v_{i} \in V$, and compare two schedules $T_{1}$ and $T_{2}$ using a preference function that maps a schedule to a utility value $f: T \rightarrow \mathcal{R}^{+}$(Khatib et al., 2001).

The relaxation problems presented here, called Controllable Conditional Temporal Problems (CCTPs), are closely related to CTPs; however, there are two important differences.

First, CCTPs assume that all real-valued variables (events) and finite-domain variables (choices) are controllable, meaning that they are under the control of the agent. Consequently, to determine the consistency of a CCTP, it is sufficient to have a single interpretation that satisfies all activated constraints, where an interpretation is a set of assignments to all discrete and continuous variables.

Second, CCTP extends the domains of discrete variables from binary to finite domain. In addition, it allows the discrete variables to be guarded by a set of assignments, which are called the guard assignments for the variable.

Definition 2. A CCTP is a 9-tuple $\left\langle P, Q, V, E, R E, L_{e}, L_{p}, f_{p}, f_{e}\right\rangle$, where:

- $P$ is a set of controllable guarded finite domain variables;

- $Q$ is the collection of assignments to P;

- $V$ is a set of events representing designated time points;

- E is a set of guarded temporal constraints between pairs of events $v_{i} \in V$;

- $R E \subseteq E$ is a set of relaxable constraints whose temporal bounds can be relaxed;

- $L_{e}: E \rightarrow 2^{Q}$ is a function that attaches conjunctions of assignments in $Q, q_{i} \in Q$, to some temporal constraint $e_{i} \in E$;

- $L_{p}: P \rightarrow 2^{Q}$ is a function that attaches conjunctions of assignments in $Q, q_{i} \in Q$, to some discrete variable $p_{i} \in P$;

- $f_{p}: Q \rightarrow \mathcal{R}^{+}$is a function that maps each assignment to every controllable discrete variable, $q_{i} \in Q$, to a positive reward value;

- $f_{e}:\left(e_{i}, e_{i}^{\prime}\right) \rightarrow \mathcal{R}^{+}$is a function that maps the relaxation to one relaxable temporal constraint $e_{i} \in R E$, from $e_{i}$ to $e_{i}^{\prime}$, to a positive cost value.

Here we refer to the deep-sea exploration scenario as a grounded example for the definition. In the CCTP model of the scenario, $P$ contains two finite domain variables $A M$ and $M S$. Their domain values $Q$ contains two sets: $\{A, B\}$ for $A M$ and $\{X, Y, Z\}$ for $M S$. The set $V$ of the CCTP contains all events in Table 1, while the set $E$ contains all constraints in Table 2. The relaxable constraints, $R E$, consists of $C_{1}$ through $C_{5}$ and $C_{17}$. Labeling function $L_{e}$ attaches assignments of $A M$ and $M S$ to guarded constraints in $E$, which are constraints $C_{1}$ through $C_{16}$ in this example. Finally, the assignment reward function, $f_{p}$, 
is defined over the five variable assignments and associates them with positive real values: $A M \leftarrow A(40), A M \leftarrow B(100), M S \leftarrow X(73), M S \leftarrow Y$ (80) and $M S \leftarrow Z$ (47). While the relaxation cost function, $f_{e}$, is defined over the six constraints in $R E$, as shown in Figure 3 .

To allow the continuous relaxation for an over-constrained temporal problem, we include relaxable temporal constraints in the definition of CCTP $(R E)$, similar to the soft constraints in a Simple Temporal Problem with Preferences (Rossi, Sperduti, Venable, Khatib, Morris, \& Morris, 2002). We do not use a disjunctive set of temporal bounds for soft constraints. Instead, the constraint is soft in that its lower or upper bounds can be relaxed continuously at the price of increasing cost. The cost is defined over the degree of relaxation made to the lower and upper bounds. Continuous relaxation provides greater flexibility in resolving over-constrained temporal problems: it does not limit our options to the predefined alternative temporal bounds, and allows us to weaken the constraint to the minimum extent necessary.

In the CCTP formulation, there is one reward function, $f_{p}$, and one cost function $f_{e} \cdot f_{p}$ is defined over the assignments to controllable discrete variables $q_{i} \in Q$. Each assignment is mapped to a positive reward value, such as $M S \leftarrow X: 73$. The larger the number is, the more preferred the choice will be. $f_{e}$ is defined over relaxable constraints. The cost of relaxing an upper bound constraint $E_{i j}: v_{j}-v_{i} \leq u_{i j}$ from $u_{i j}$ to $u_{i j}^{\prime}$ is $f_{e i j}\left(u_{i j}^{\prime}\right)$. Figure $3 \mathrm{~b}$ shows an example function defined over $u_{i j}^{\prime}$.

The cost function for temporal constraints that restrict the lower bounds between two events is $f_{e i j}\left(l_{i j}^{\prime}\right)$. This is illustrated in Figure 3a. We assume that the user always prefers smaller relaxations: the values in the original constraints are equally and maximally preferred, and the preferences decreases as one moves away from the original bounds. The motivation of this assumption is that people generally prefer to minimize the perturbations to the original requirements, and penalize larger deviations from them. Therefore, all $f_{e}$ functions must be monotonically increasing, and equal to 0 when there is no relaxation. $f_{e}$ can be viewed as a semi-convex (Khatib et al., 2001) function with a segment of zero cost when there is no relaxation. This assumption simplifies our relaxation process, as the tightest relaxation will always result in the lowest cost. For relaxable simple temporal constraints, two separate cost functions are required for the lower and upper bounds.

Definition 3. A solution to a CCTP is a pair $\left\langle A, R_{e}\right\rangle$ such that all activated constraints are temporally consistent, where:

- $A$ is a set of assignments, $A \subseteq Q$, that leaves no variable unassigned;

- $R_{e}$ is a set of temporal relaxations for some constraints in $R E$.

where a temporal relaxation is defined as a tuple, $\left\langle e, r_{L}, r_{U}\right\rangle$, as the following:

- $e$ is a constraint in $R E$;

- $r_{L}$ is a weakened lower bound for $e$ and $r_{L} \leq e_{\text {lowerbound; }}$;

- $r_{U}$ is a weakened upper bound for $e$ and $r_{U} \geq e_{\text {upperbound }}$. 
The utility of a solution is computed by subtracting the relaxation cost from the assignment reward: $\sum_{p_{i}} f_{p_{i}}\left(p_{i} \leftarrow\right.$ value $\left._{i}\right)-\sum_{e_{i}} f_{e_{i}}\left(e_{i} \rightarrow e_{i}^{\prime}\right)$. For example, Solution 1 in Table 3 consists of two assignments and two relaxations: assignment $A M \leftarrow B$ and $M S \leftarrow Y$ have reward of 100 and 80, while the cost of relaxing $C_{2}$ and $C_{17}$ are 6 and 2.5, respectively. Hence the utility value of this solution is 171.5 , which is computed by summing up the rewards, and subtracting the cost of relaxing $C_{4}$ and $C_{17}$ from it. Given a CCTP, the most preferred relaxation to it is the one with the highest utility value according to $f_{p}$ and $f_{e}$.

Compared to the Temporal Constraint Satisfaction Problems (TCSPs) and Disjunctive Temporal Problems (DTPs) formulation (Dechter et al., 1991; Stergiou \& Koubarakis, 1998a), whose constraints are disjunctions of possible simple temporal constraints, CCTP is often more compact for real-world scenarios since it allows a sequence of temporal constraints to be conditioned on choices. The Temporal Networks with Alternatives (TNA) formulation, introduced by Barták and Cepek (2007), also address this issue by introducing the concepts of parallel and alternative processes. However, due to its nested network structure, it is often less efficient in encoding constraints that are conditioned on assignments to two independent variables, such as the traversals between asphalt mound and methane seep sites.

Note that CCTP is similar, though different in notation, to the Optimal Conditional Simple Temporal Problem (OCSTP) formulation, which was introduced by Effinger (2006). OCSTP and CCTP are equally expressive for consistency problems. OCSTP encodes temporal constraints as the domain values of discrete variables, and its relaxations are represented by additional domain values. This makes it difficult to encode the relaxable temporal constraints using an OCSTP formulation. We chose CCTP for relaxation problems because of its compact representation of constraint relaxations: consistency can be restored by relaxing the lower or upper bounds of relaxable temporal constraints.

\subsection{Controllable Conditional Temporal Problems with Uncertainty}

Uncertainty is commonly encountered in temporal scheduling and planning problems, and can often lead to over-constrained situations. In Rich's mission described earlier, the traversal times between locations are often non-deterministic. When applying the deterministic formulation to model such problems, their relaxations may fail since they only satisfy a subset of the possible outcomes for the uncertain durations. Hence, we introduced the Controllable Conditional Temporal Problem with Uncertainty (CCTPU) formalism; an extension to the CCTP formulation, for modeling relaxation problems with uncertain duration. Their definitions differ only in the terms of constraints: in addition to temporal constraints with controllable durations, a CCTPU may also contain uncertain durations. This is similar to to the generalization from STPPs to the Simple Temporal Problems with Preferences and Uncertainty (STPPUs) framework (Rossi, Venable, \& Yorke-Smith, 2006), which adds support for modeling contingent events and uncertain durations.

We use Rich's mission to illustrate the modeling of relaxation problems with uncertain duration. Table 2 repeats all the constraints for his mission. Note that Constraints $C_{6}$ through $C_{16}$ are highlighted in bold: they are uncontrollable temporal durations and encode the traversal times between locations. Their temporal bounds indicate the domain of the random outcomes. The survey times remain deterministic as we can give a deadline for 
departure from each survey location. Similar to CCTP, CCTPU can also be visualized using a node-arc graph, in which uncontrollable durations are represented by double arcs (Figure 4).

\begin{tabular}{|lll|}
\hline \multicolumn{3}{|c|}{ Constraints and Uncertain Durations (in minutes) } \\
\hline$C_{1}(R): A_{L^{-}} A_{A} \in[50,60]$ & $\mathbf{C}_{\mathbf{6}}: A_{A}-S \in[45,65]$ & $A M \leftarrow A$ \\
$C_{2}(R): B_{L}-B_{A} \in[45,60]$ & $\mathbf{C}_{\mathbf{7}}: B_{A^{-}} S \in[30,50]$ & $A M \leftarrow B$ \\
$C_{3}(R): X_{L}-X_{A} \geq 60$ & $\mathbf{C}_{\mathbf{8}}: E-X_{L} \in[28,35]$ & $M S \leftarrow X$ \\
$C_{4}(R): Y_{L}-Y_{A} \geq 65$ & $\mathbf{C}_{\mathbf{9}}: E-Y_{L} \in[30,32]$ & $M S \leftarrow Y$ \\
$C_{5}(R): Z_{L}-Z_{A} \geq 100$ & $\mathbf{C}_{\mathbf{1 0}}: E-Z_{L} \in[50,60]$ & $M S \leftarrow Z$ \\
\hline $\mathbf{C}_{\mathbf{1 1}}: X_{A^{-}} A_{L} \in[51,54]$ & $A M \leftarrow A$ and $M S \leftarrow X$ \\
$\mathbf{C}_{\mathbf{1 2}}: Y_{A}-A_{L} \in[42,45]$ & $A M \leftarrow A$ and $M S \leftarrow Y$ \\
$\mathbf{C}_{\mathbf{1 3}}: Z_{A}-A_{L} \in[30,55]$ & $A M \leftarrow A$ and $M S \leftarrow Z$ \\
$\mathbf{C}_{\mathbf{1 4}}: X_{A}-B_{L} \in[22,24]$ & $A M \leftarrow B$ and $M S \leftarrow X$ \\
$\mathbf{C}_{\mathbf{1 5}}: Y_{A}-B_{L} \in[21,25]$ & $A M \leftarrow B$ and $M S \leftarrow Y$ \\
$\mathbf{C}_{\mathbf{1 6}}: Z_{A}-B_{L} \in[30,35]$ & $A M \leftarrow B$ and $M S \leftarrow Z$ \\
\hline$C_{17}(R): E-S \in[0,180]$ & \\
\hline
\end{tabular}

Table 4: Temporal constraints and uncertain duration in Rich's mission CCTPU

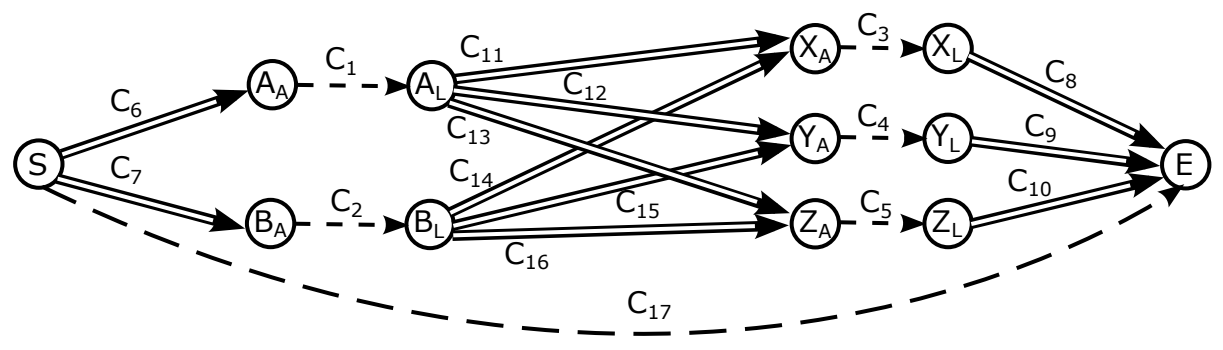

Figure 4: A graphical representation of Rich's mission CCTPU

Similar to the earlier example, without any relaxations, there is no solution that can satisfy all of the requirements in Rich's mission. If we use the CCTP formalism to model this over-constrained problem, one solution would be to visit site $\mathrm{B}$ and $\mathrm{X}$ while extending the mission by 5 minutes (Figure 5). However, it does not account for the uncontrollable traversal times: the solution is very likely to fail during the mission, since it has no margin to absorb any delay in the traversal between locations. Next, we present two solutions generated for the CCTPU model, which are based on two execution strategies that take the uncertainty into consideration. The first strategy, called Strong Controllability, comes up with a schedule of activities before starting the plan, which ensures success for all uncontrollable durations. The second execution strategy, called Dynamic Controllability, instead observes these uncertain outcomes along the way, and makes more informed decisions about scheduling each activity.

The second solution is computed based on strong controllability (Figure 6). It extends the mission time to 209 minutes, and decreases the lower bound of the survey time at $B$ to account for the uncertainty in the traversal between the ship and site $B$. This solution 


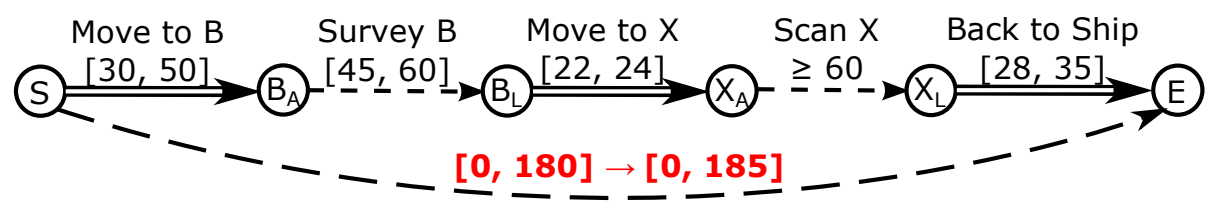

Figure 5: Consistent relaxation for Rich's mission

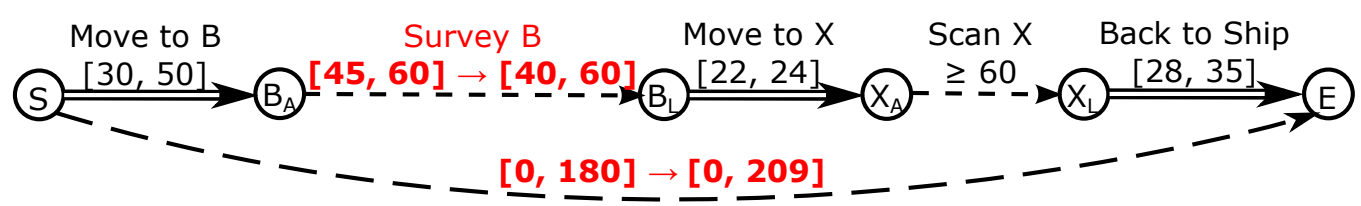

Figure 6: Strongly controllable relaxation for Rich's mission

enables a schedule that satisfies Rich's requirements while being robust to the uncertain durations.

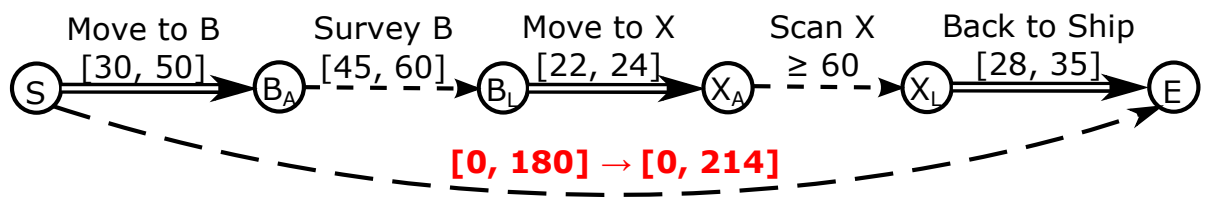

Figure 7: Dynamically controllable relaxation for Rich's mission

The third and final solution is computed based on dynamic controllability (Figure 7). Unlike the strong controllability solution, it does not need to decrease the lower bound of survey time at $B$ to account for the uncertain traversal times, and hence is less conservative than the second solution, while still being safe. The solution enables a dynamic schedule on the fly instead of a static schedule beforehand: the times of leaving site $B$ and $X$ will depend on the actual traversal times.

\subsubsection{Definitions}

Simple Temporal Networks with Uncertainty (STNUs) (STNUs, Vidal \& Fargier, 1999) have been widely used to model temporal problems with uncertain durations. They are extension to STNs by adding a new class of constraint: uncertain duration. The duration is defined by a random variable between its lower and upper bounds and cannot be freely assigned. Formally, this structure is defined as the following:

Definition 4. A STNU $N=\left\langle V_{a}, V_{r}, E_{f}, E_{u}\right\rangle$, extends the $S T N$ definition with new types of events and constraints associated with the uncertain duration:

- activated events $v_{i} \in V_{a}$, whose times are assigned by the agent;

- received events $r_{i} \in V_{r}$, whose times are assigned by external world;

- free constraints $e_{i} \in E_{f}$, which are of type $v_{x}-v_{y} \in\left[l_{x y}, b_{x y}\right]$ where $v_{x}, v_{y}$ are events. 
- uncertain durations $u_{x y} \in E_{u}$, where $u_{x y} \in\left[l_{x y}, b_{x y}\right]$ describes the difference in time between one received event $r_{y} \in V_{r}$ and one activated event $v_{x} \in V_{a}$, such that $\left(r_{y}-\right.$ $\left.v_{x}\right)=u_{x y}$; or between two received events $r_{y}, r_{x} \in V_{r}$, such that $\left(r_{y}-r_{x}\right)=u_{x y}$. Uncertain durations are not assigned by the agent, and can take any value in the bounded interval.

The STNU extends the STN representation with uncertainty. Note that the uncertainty representation is not probabilistic: the uncertain durations are not associated with probability distributions. Thus, we can not reason over the likelihood of outcomes for the uncertain durations. We must instead guarantee constraint satisfaction given any outcome for the uncertain duration within the interval.

The solution to a STNU is an execution strategy that satisfies all constraints regardless of the outcomes of uncertain duration. The existence of such a strategy is characterized by the controllability, instead of consistency, of the STNU. There are three types of controllability (Vidal \& Fargier, 1999): Strong, Dynamic, and Weak. Each type has a different assumption about the time when the outcomes of uncertain duration become available. In this paper, we focus on the first two types, strong and dynamic controllability, which assume that no outcome is known prior to the execution. Strong controllability requires a predetermined schedule which satisfies all constraints regardless of the outcomes of the uncertain durations, whereas dynamic controllability requires a policy for scheduling as observations of uncertain durations become available. Intuitively, dynamic controllability is more flexible as it makes use of information gained during execution.

Definition 5. A Simple Temporal Problem with Uncertainty, STPU, is a problem formulation using the STNU representation.

- Given a STNU $N=\left\langle V_{a}, V_{r}, E_{f}, E_{u}\right\rangle$, find an execution policy to events in $V_{a}$ such that all constraints in $E_{f}$ are satisfied regardless of the outcomes of durations in $E_{u}$.

The STPU formalism has been extended with disjunctions and conditional constraints to handle more real-world scheduling and planning problems. Venable and Yorke-Smith (2005) and Peintner, Venable, and Yorke-Smith (2007) introduced the Disjunctive Temporal Problem with Uncertainty (DTPU) formalism to permit non-convex and non-binary constraints. DTPU can be viewed as an extension to the deterministic Disjunctive Temporal Problem formalism (Stergiou \& Koubarakis, 1998b) with uncertain durations. It allows the expression of disjunctive constraints, and enables the agent to choose between alternatives. Hunsberger, Posenato, and Combi (2012) introduced the Conditional Temporal Problems with Uncertainty (CTPUs) formalism to model problems with both uncontrollable duration and observations. We introduce CCTPU formalism to permit the description of more general scheduling and relaxation problems for scenarios with uncertain durations. It is equally expressive as DTPUs and allows more compact encodings of conditional decision variables and constraints. On the other hand, we are only concerned with controllable decision variables. This makes CCTPUs strictly simpler to solve compared to CTPUs, in that those tasks may require the enumeration of all scenarios that are dependent on uncontrollable variables.

Formally, the CCTPU formulation extends the CCTP definition with three additional elements. 
Definition 6. A CCTPU contains all elements in a CCTP, plus $V_{r}, E_{u}$ and $R E_{u}$, where:

- $V_{r} \subseteq V$ is a set of received events. $V \backslash V_{r}$ is the set of all activated events;

- $E_{u} \subseteq E$ is a set of guarded uncertain duration between pairs of activated and received events. The temporal bounds of these duration indicate the domain of possible values of the random outcomes. $E \backslash E_{u}$ is the set of all free constraints;

- $R E_{u} \subseteq E_{u}$ is a set of relaxable uncertain durations whose bounds can be tightened, and $R E_{u} \subseteq R E$.

The cost function is generalized to include both free constraints and uncertain duration: $f_{e}:\left(e_{i}, e_{i}^{\prime}\right) \rightarrow \mathcal{R}^{+}$is a function that maps the following to a non-negative cost.

- the relaxation of a free constraint, $e_{i} \rightarrow e_{i}^{\prime}, e_{i} \in R E \backslash R E_{u}$;

- or the tightening of an uncertain duration, $e_{i} \rightarrow e_{i}^{\prime}, e_{i} \in R E_{u}$;

As can be seen in the definition, we also generalize the concept of relaxations to include uncertain durations. To resolve a conflict by relaxing free constraints, we will either increase its upper bound or reduce its lower bound. On the other hand, we shrink the uncertainty for uncertain duration in a conflict: we may resolve the conflict by increasing the lower bound and/or decreasing the upper bound of its uncertain duration. Usually, uncertain duration is used to reserve some flexibility for the agents or the environment in executing their tasks. A tighter duration means less flexibility for them, but also imposes less restriction on the solution to the temporal problem. We will give more insights into the relation between uncertain durations and conflict resolutions in the approach section.

Similar to CCTP, the solution to a CCTPU is defined as a 3-tuple $\left\langle A, R_{e}, R_{u}\right\rangle$, where:

- $A$ is a complete set of assignments to variables in $P$ that leaves no variable unassigned.

- $R_{e}$ is a set of relaxations for some free constraints in $R E \backslash R E_{u}$.

- $R_{u}$ is a set of tightenings for some uncertain durations in $R E_{u}$.

A feasible solution to a CCTPU provides a grounded and controllable STPU. We separate the solutions into two categories: strongly controllable and dynamically controllable. This is based on the type of execution strategies a solution can enable.

- A strongly controllable solution makes the resulting STPU strongly controllable. That is, the relaxation enables an execution strategy with a firm schedule for all events.

- A dynamically controllable solution makes the resulting STPU dynamically controllable, for which a dynamic execution strategy that reacts in real time to observations of uncertain duration and meets all constraints can be derived.

Note that a strongly controllable solution is also a dynamically controllable solution, since strong controllability is more restrictive than dynamic controllability. Due to the flexibility of dynamic controllability, there is usually a greater solution space to explore for this type of problem. 


\subsection{Conditional Probabilistic Temporal Problems with Chance-Constraints}

In many situations, modeling the uncertainty in temporal duration with a set-bounded representation is overly conservative, resulting in a loss of schedule utility. Chance-constrained formalisms, such as chance-constrained probabilistic Simple Temporal Problems (cc-pSTPs), address over-conservatism by imposing bounds on risk, while maximizing utility subject to these risk bounds. On the other hand, when we are dealing with probabilistic uncertain duration, the relaxation problem becomes more challenging, since we are making trade-offs between not only timing requirements, but also risk taken. In the rest of this section, we present an extended formalism to CCTPs, the chance-constrained probabilistic Controllable Conditional Temporal Problems (cc-pCCTPs) for modeling relaxation problems with chance constraints.

We again use Rich's mission as an example to illustrate the modeling. To simplify the example, we remove the asphalt mound site survey, leave only the methane seep sites to visit, and make the traversal duration controllable. The periodic methane seep at $\mathrm{X}$ is likely to occur at around 1:00PM, following a normal distribution with a standard deviation of 30 minutes. The seep at $\mathrm{Y}$ will occur at around 1:30PM and follows a normal distribution with a standard deviation of 50 minutes. As described before, Sentry leaves the ship at 11:00AM, and needs to arrive at the site before the start of the methane seep. In addition, at least 30 minutes is required for traversing to the site, and 45 minutes for scanning. As before, Rich wants the mission to complete in 3 hours, with less than $5 \%$ risk of violating any constraint, such as returning late or missing the event. We can capture this problem using a cc-pCCTP (Figure 8).

\begin{tabular}{|llll|}
\hline \multicolumn{4}{c|}{ Constraints and Uncertain Durations (in minutes) } \\
\hline$C_{1}$ & $X_{A^{-}} S>[45,+\infty]$ & $M S \leftarrow X$ & Traversal to methane seep site X \\
$C_{2}$ & $X_{s p^{-}} X_{A} \in[0,+\infty]$ & $M S \leftarrow X$ & Wait for seep at site X \\
$C_{3}(\mathrm{R})$ & $X_{L^{-}} X_{s p} \in[50,60]$ & $M S \leftarrow X$ & Scanning at site X \\
$C_{4}$ & $E-X_{L} \in[45,+\infty]$ & $M S \leftarrow X$ & Traversal from site X back to ship \\
$\mathbf{C}_{\mathbf{5}}$ & $X_{s p}-S \sim N(120,30)$ & $M S \leftarrow X$ & Seep start time at X \\
$C_{6}$ & $Y_{A}-S \in[30,+\infty]$ & $M S \leftarrow Y$ & Traversal to methane seep site Y \\
$C_{7}$ & $Y_{s p}-Y_{A} \in[0,+\infty]$ & $M S \leftarrow Y$ & Wait for seep at site Y \\
$C_{8}(\mathrm{R})$ & $Y_{L}-Y_{s p} \in[45,60]$ & $M S \leftarrow Y$ & Scanning at site Y \\
$C_{9}$ & $E-Y_{L} \in[30,+\infty]$ & $M S \leftarrow Y$ & Traversal from site Y back to ship \\
$\mathbf{C}_{\mathbf{1 0}}$ & $Y_{s p}-S \sim N(150,50)$ & $M S \leftarrow Y$ & Seep start time at Y \\
$C_{11}(\mathrm{R})$ & $E-S \in[0,180]$ & & Mission duration \\
\hline
\end{tabular}

Table 5: Temporal constraints and uncertain duration in Rich's mission cc-pSTP

After evaluating all the requirements, BCDR determines that no dynamic execution policy exists that meets all requirements. It engages Rich and starts presenting relaxations that can restore the feasibility of Rich's problem. The first one asks Rich to extend the mission from 3 hours to 4 hours and 26 minutes, which is robust for surveying the methane seep at site $\mathrm{X}$ if it occurs between 11:45AM and 1:51PM (Figure 9). The probability of failure is determined by analyzing the assumptions on the uncertain duration: the proba- 


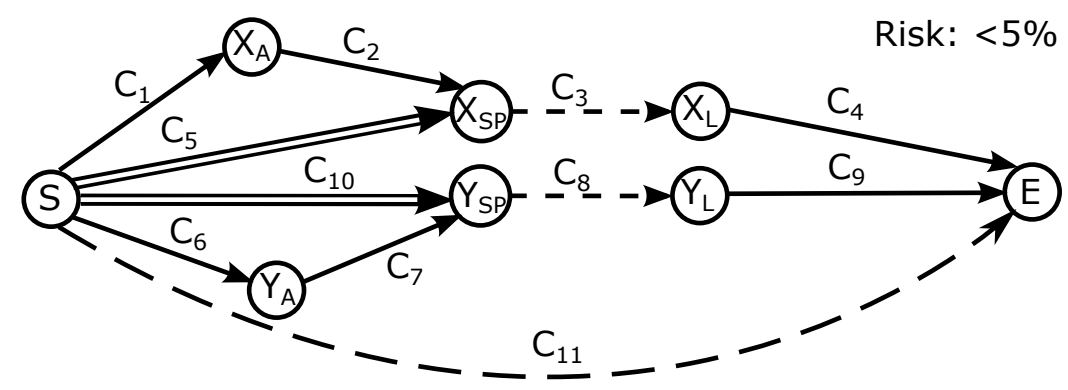

Figure 8: The cc-pCCTP model for Rich's mission

bilistic duration $C_{5}$ is turned into a set bounded one with bounds [45,171], and the network is checked to be dynamically controllable against all possible outcomes within the range.

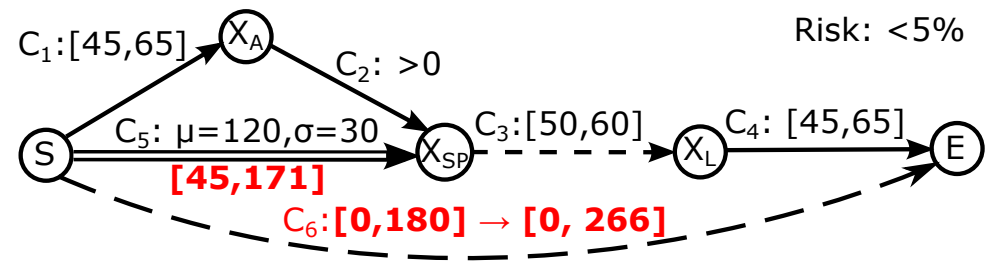

Figure 9: First relaxation for Rich's mission

However, Rich rejects the solution and tells BCDR that the mission duration can be at most 4 hours, since the subsequent mission cannot be shortened by more than an hour. $\mathrm{BCDR}$ incorporates this new requirement and generates another relaxation, which requires Rich to accept an increased probability of failure, from $5 \%$ to $20.85 \%$. This allows the mission to be completed in 4 hours, but cannot account for methane seep that occurs after 1:25PM (Figure 10).

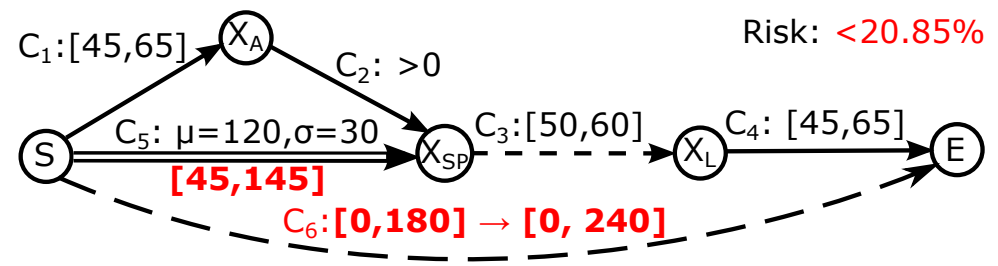

Figure 10: Second relaxation for Rich's mission

Rich rejects the solution again and tells BCDR that he cannot take more than $5 \%$ risk. BCDR again incorporates this new constraint and generates the third relaxation, which asks Rich to reduce the survey time at site $\mathrm{X}$ to 24 minutes. This allows the mission to be completed in 4 hours, while being robust to methane seep that takes place between 11:45AM and 1:51PM (Figure 11). 


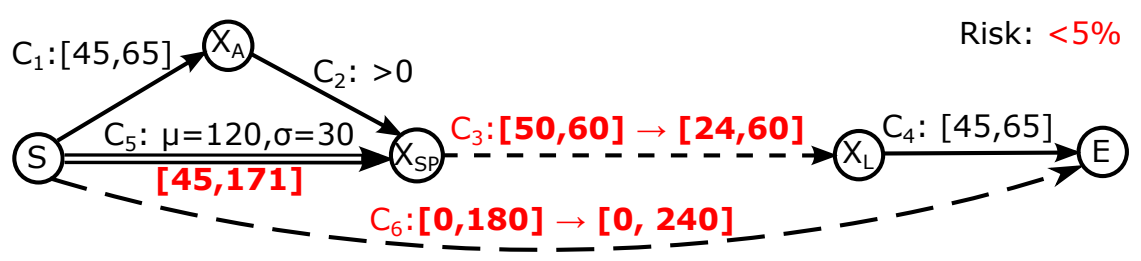

Figure 11: Third relaxation for Rich's mission

\subsubsection{Definitions}

Similar to the STNU formalism, cc-pSTP is also an extension to the Simple Temporal Problems (STPs) formulation. In addition to the simple temporal constraints in STPs, it adds two new types of constraints to the problem: probabilistic temporal constraints for modeling uncertain durations, and chance constraints for specifying the acceptable level of risk. Compared to the set-bounded uncertain durations used in STNUs, the probabilistic representation of uncertain durations allows cc-pSTPs to more accurately model uncertainty in real world activities. In addition, the chance constraint supports a quantified bound on risk taken to be specified, which is more flexible and intuitive than the criteria of controllability. It can be viewed as a generalization of the notion of controllability: instead of a binary outcome between $100 \%$ guarantee of success or nothing, the users can ask for any bound between $[0,100 \%]$ on the probability that a temporal network is executable.

Chance-constraints have been studied in the operations research literature, traditionally as probabilistic guarantees over satisfaction of individual or conjunctions of constraints (Kall, 1976). In this work, we generalize the definition of chance-constraints to include disjunctions over constraints as well. It allows us to choose between multiple options to find one which meets our safety requirements.

In the definition below, we use the standard definition of random variables from probability theory (Durrett, 2010). Intuitively, when describing outcomes of random variables, we can imagine sampling $\omega$ from a sample space $\Omega$. The outcomes of random variables can be generated via functions $\mathbf{f}(\omega)$. Further, we have a probability measure $P$, which takes a subset $S \subseteq \Omega$ and gives the probability of samples being in $S$.

A chance-constraint is rigorously defined as follows:

Definition 7. Consider a constraint program with decision variables $\mathbf{x}$, and a set of random variables $\mathbf{f}(\omega)$ with probability measure $P$, sample space $\Omega$, and $\omega \in \Omega$.

Let the set of constraints be defined over decision variables and random variables as

$$
C=\bigvee_{i \in I} \bigwedge_{j \in J_{i}} A_{j}(\mathbf{x}, \mathbf{f}(\omega)) \geq b_{j}
$$

where each $A_{j}$ is a measurable function, I can be thought of as a set of distinct scenarios, and $J_{i}$ can be thought of as a listing of the set of constraints required to hold in each scenario.

$A$ chance-constraint is a tuple $\langle C, \Delta\rangle$, requiring:

$$
P(C \text { satisfied }) \equiv P\left(\left\{\omega \mid \bigvee_{i \in I} \bigwedge_{j \in J_{i}} A_{j}(\mathbf{x}, \mathbf{f}(\omega)) \geq b_{j}\right\}\right) \geq \Delta
$$


As an example, consider a chance-constraint for Rich's mission in Figure 8. There are two distinct scenarios, in which sites $\mathrm{X}$ or $\mathrm{Y}$ are visited respectively. For the visit to site $\mathrm{X}$, the constraints which must hold are $C_{1}, C_{2}, C_{3}, C_{4}$, and $C_{11}$, and for the visit to site Y, the constraints which must hold are $C_{6}, C_{7}, C_{8}, C_{9}$, and $C_{11}$. Note that, of the constraints, only $C_{2}, C_{3}, C_{7}$, and $C_{8}$ involve uncontrollable time points. Thus, we have our set of constraints $C$ in the chance constraint

$$
\begin{array}{r}
C=\left(\left(X_{s p}-X_{A} \geq 0\right) \wedge\left(X_{L}-X_{s p} \geq 50\right) \wedge\left(-X_{L}+X_{s p} \geq-60\right)\right) \vee \\
\left(\left(Y_{s p}-Y_{A} \geq 0\right) \wedge\left(Y_{L}-Y_{s p} \geq 45\right) \wedge\left(-Y_{L}+Y_{s p} \geq-60\right)\right)
\end{array}
$$

While the definition here is presented for constraint systems in disjunctive normal form, this is a general representation with conversions via De Morgan's laws, and is sufficient for the chance-constrained problem definitions presented below.

The cc-pCCTP formalism extends CCTPU with probabilistic uncertain durations and chance constraints. Here, we first repeat the definition of cc-pSTP, as presented by Fang et al. (2014), for reference.

Definition 8. A cc-pSTP is a pair $\left\langle N^{+}, \Delta_{t}\right\rangle$, where:

- $N^{+}$is a probabilistic Simple Temporal Network (pSTN), defined as a 4-tuple $\left\langle V_{a}, V_{r}, E_{f}, E_{d}\right\rangle$, where:

- $V_{a}, V_{r}, E_{f}$ are defined as for STNU; and

- $E_{d}$ is a set of probabilistic uncertain durations. Each $d_{x y} \in E_{d}, d_{x y}: \Omega \rightarrow \mathbb{R}$ is a random variable describing the difference $(y-x)$ between an activated event $x \in V_{a}$ and a received event $y \in V_{r}$.

- $\left\langle E_{f}, 1-\Delta_{t}\right\rangle$ is the chance constraint that sets the upper bound on the risk of failure, for the set of requirement constraints $E_{f}$ in $N^{+}$.

In the cc-pSTP, the set of constraints is described by $E_{f}$, which are difference constraints between elements in $V_{r}$ and $V_{a}$ to be satisfied, given the outcomes of $E_{d}$. This corresponds to $C$ in Definition 7.

Definition 9. A cc-pSTP solution is a pair $\left\langle N_{g}, S_{x}\right\rangle$, where:

- $N_{g}$ is a grounded STNU of the cc-pSTP. It replaces all probabilistic durations in the cc-pSTP with set-bounded uncertain durations, which specify the allocated risk over them: the lower and upper bounds allocated for each probabilistic duration indicate the range of outcomes covered. The total probability of uncovered outcomes across all probabilistic durations must be smaller than the chance constraint.

- $S_{x}$ is an execution strategy for $N_{g}$. It covers all controllable events in the cc-pSTP, and is controllable with respect to $N_{g}$. 
Given a cc-pSTP, $A$, if we execute the controllable events using $S_{x}$ in its solution, the chance of violating any temporal constraints in $A$ is guaranteed to be less than $\Delta_{t}$. The policy $S_{x}$ could be a static schedule (with a strongly controllable $N_{g}$ ), or a dynamic execution policy (with a dynamically controllable $N_{g}$ ). If a cc-pSTP is over-constrained, no solution exists that can meet all temporal constraints within the risk bound. In other words, there is no $N_{g}$ that is controllable and takes less risk than the chance constraint. This occurs when the user specifications are too restrictive, for example when the desired time bounds are too tight, or when the user is overly cautious in setting the chance constraint. These problems can be resolved through temporal or chance constraint relaxations: they are trade-offs between weakening over chance and temporal constraints for the users. We thus define a relaxable version of the cc-pSTP formulation, which allows some of its constraints to be relaxed at a cost to allow a feasible solution.

Definition 10. A relaxable cc-pSTP contains all elements from a cc-pSTP plus four additional elements, $R E, f_{e}, r \Delta_{t}$ and $f_{\Delta}$, where:

- $R E$ is a set of requirement constraints whose temporal bounds can be relaxed, $R E \subseteq$ $E_{f}$.

- $f_{e}:\left(e_{i j}, e_{i j}^{\prime}\right) \rightarrow \mathbb{R}^{+}$is a function that maps the relaxation of a relaxable constraint, $e_{i j} \rightarrow e_{i j}^{\prime}$ where $e_{i j} \in R E$, to a positive cost value.

- $r \Delta_{t} \in[T, F]$ is a boolean value that indicates if the chance constraint can be relaxed.

- $f_{\Delta}:\left(\Delta_{t}, \Delta_{t}^{\prime}\right) \rightarrow \mathbb{R}^{+}$is a function that maps the relaxation of the chance constraint, $\Delta_{t} \rightarrow \Delta_{t}^{\prime}$ where $\Delta_{t} \leq \Delta_{t}^{\prime} \leq 1$, to a positive cost value.

Definition 11. A valid resolution for an over-constrained cc-pSTP, A, is a 3-tuple $\left\langle R_{e}, \Delta_{t}^{\prime}, N_{\text {alloc }}\right\rangle$, where:

- $R_{e}$ is a set of relaxations (in terms of relaxed lower and upper bounds) to constraints in $R E$ of $A$.

- $\Delta_{t}^{\prime}$ is a relaxation for $\Delta_{t}$ of $A$, and $\Delta_{t}^{\prime} \geq \Delta_{t}$.

- $N_{\text {alloc }}$ is a STNU generated from A by grounding all probabilistic durations with fixed lower and upper bounds.

such that $N_{\text {alloc }}$ is controllable and covers more than $1-\Delta_{t}^{\prime}$ of the uncertain durations' outcomes.

Given an over-constrained cc-pSTP, there is usually more than one valid resolution to it due to the continuous property of temporal and chance constraint relaxations. It is important to prioritize the resolutions and enumerate only preferred ones of lower cost for the users. In addition, finding a good resolution usually requires a considerable amount of negotiation since the users may not have encoded all their constraints in the input problem. BCDR needs to learn about them through the interaction before reaching an agreement with the user. 
Given that we are also interested in problems which features discrete choices in our course of actions, we also define a chance-constrained analogue to the CCTPU, the chanceconstrained probabilistic CCTP (cc-pCCTP).

Definition 12. A cc-pCCTP contains all elements in a CCTPU (Definition 6), with the following differences:

- instead of $E_{u} \subseteq E$, a set of guarded uncertain duration between pairs of activated and received events in $C C T P U$, the cc-pCCTP features a set of probabilistic uncertain durations $E_{d}$. Each $d_{x y} \in E_{d}$ is a random variable describing the difference $(y-x)$ between an activated event and a received event, where $x \in V_{a}$ and $y \in V_{r}$;

- similar to cc-pSTP, a cc-pCCTP includes $\left\langle E_{f}, 1-\Delta_{t}\right\rangle$ the chance constraint that sets the upper bound on the risk of failure, for the set of requirement constraints $E_{f} \subseteq E$;

- a cc-pCCTP includes a boolean value $r \Delta_{t} \in[T, F]$ that indicates if the chance constraint can be relaxed; and

- The cost function is adapted for the chance constraint with the addition of $f_{\Delta}$ : $\left(\Delta_{t}, \Delta_{t}^{\prime}\right) \rightarrow \mathbb{R}^{+}$, a function that maps the relaxation to the chance constraint, $\Delta_{t} \rightarrow \Delta_{t}^{\prime}$ where $\Delta_{t} \leq \Delta_{t}^{\prime} \leq 1$, to a positive cost value.

Intuitively, the cc-pCCTP formulation adds probabilistic uncertain durations with an associated chance-constraint, in addition to the set-bounded uncertain durations in CCTPUs. Correspondingly, the relaxation over the risk bound is encoded by the tightness of the chance constraint.

The concept of relaxing an uncertain duration is related to the concept of relaxing a chance-constraint. In finding a solution to cc-pCCTP, we are required to find an example set of set-bounded uncertain durations which cover enough probability mass to satisfy the chance constraint. A relaxation of the chance constraint allows the choice of set-bounded uncertain durations which cover a smaller probability mass, in some cases a more restrictive set of outcomes. This is analogous to the relaxation of the uncertain durations in the original CCTPUs.

\section{Approach}

In this section, we present the Best-first Conflict-Directed Relaxation algorithm, a generalization of Conflict-directed A* (Williams \& Ragno, 2002) that computes continuous relaxations for over-constrained temporal problems with multiple different types of constraints in a unified manner. As presented in Section 1, the BCDR algorithm, like CDA*, takes an iterative approach to discovering conflicting constraints and computing resolutions, in the form of relaxations. BCDR is unique in its ability to resolve through continuous relaxations, and its introduction of continuous conflicts, to focus this relaxation process.

We start this section with an overview of the conflict-directed relaxation framework, and how it applies to Controllable Conditional Temporal Problems (CCTPs). This is the simplest of the problems addressed in this paper, since all its constraints have controllable 
temporal bounds. We focus on the conflict-directed framework that allows BCDR to iteratively discover conflicts, and resolve them using both discrete and continuous relaxations.

Second, we describe an extension to the BCDR algorithm for handling temporal problems with set-bounded uncertain durations (CCTPUs). We name the extended algorithm BCDR-U, which incorporates a new conflict learning procedure for discovering conflicts with set-bounded uncertain durations. The extension in BCDR-U allows it to restore the controllability, in addition to consistency, of over-constrained temporal problems. We present two version of the extension, namely BCDR-U(SC) and BCDR-U(DC), for use with strong and dynamic controllability models.

Third, we present another extension to BCDR for resolving chance-constrained temporal problems (cc-pCCTPs). We name the extension BCDR-C, in which 'C' represents chance constraints. The key idea introduced in resolving cc-pCCTPs is to resolve infeasibility for risk-bounded scheduling problems, by allowing the risk bound, as well as the temporal constraints, to be relaxed continuously. The extension introduced by BCDR-C is a new conflict resolution procedure for handling chance constraints: in addition to relaxing temporal constraints during conflict resolution, it also weakens the risk bounds, which is expressed in the chance constraints. This new conflict resolution step allows BCDR-C to handle overly risky situations by deciding to accept more risk, and achieve a good balance between the risk taken and the temporal requirements for the users. Similar to BCDR-U, we also present two versions of the extension for different controllability models: BCDR-C(SC) for strong controllability and BCDR-C(DC) for dynamic controllability.

Finally, we discuss the modifications to BCDR that enables it to incorporate user feedbacks on the fly, and a new greedy conflict resolution technique for speeding up run-time performance on problems with certain structures. They are essential for the deployment of BCDR in user-facing applications and solving real-world problems of large scale, and are compatible with not only the consistency-based BCDR algorithm, but also its two extensions, BCDR-U and BCDR-C.

In our previous work, we have introduced these variant problems and solutions through a variety of name, namely the Best-first Conflict-Directed Relaxation (BCDR) (Yu \& Williams, 2013) for CCTPs, Conflict-Directed Relaxation with Uncertainty (CDRU) (Yu et al., 2014) for CCTPUs, and Conflict-Directed Chance constraint Relaxation (CDCR) (Yu et al., 2015) for cc-pCCTPs. In this paper we unify the problem and solution algorithms, under the names CCTP and BCDR, respectively. We will discuss the extensions to BCDR for problems with different types of constraints: BCDR for solving CCTPs, BCDR$\mathrm{U}$ with extended conflict learning for solving CCTPUs, and BCDR-C with extended conflict resolution for solving cc-pCCTPs.

\subsection{BCDR: Computing Continuous Relaxations}

The Best-first Conflict-Directed Relaxation algorithm was first presented by Yu and Williams (2013) for enumerating the relaxations to a CCTP in best-first order. It adapts and extends the Conflict-Directed A* (CD-A*) algorithm (Williams \& Ragno, 2002), first developed for constraint optimization problems, with applications to hardware diagnosis, reconfiguration, repair and temporal planning. CD-A* enumerates the best solutions to discrete domain CSPs according to an objective function, and guides the search using conflicts learned from 
inconsistent sets of assignments. Once detected, a conflict is used to prune the search space by extending each partial candidate with alternative resolutions.

Similar to CD-A*, once a conflict in a CCTP is detected, BCDR uses it to prune the search space by extending each partial candidate with alternative choices, and continuous relaxations for the temporal bounds of episodes in it. Therefore, BCDR is able to explore alternative subproblems of the CCTP and its continuous relaxations simultaneously. An overview of the BCDR algorithm with the continuous relaxation extension is given in Algorithm 1.

To resolve a CCTP using the conflict-directed strategy, we have to first generalize the conflict representation to a hybrid one that includes both discrete variables and temporal constraints. To resolve these hybrid conflicts, we compute both discrete and continuous constituent relaxations to the conflict. Like CD-A*, BCDR takes an $\mathrm{A}^{*}$ search strategy by evaluating each partial candidate using an admissible heuristic function and expanding the search tree in best-first order. The first relaxation found is guaranteed to be the best one.

The key of this conflict-directed approach is to explore the search space using two types of expansions: Expand on an unassigned variable and Expand on an unresolved conflict. The first expansion guides the search into unexplored regions, and the second expansion keeps the search away from known infeasible regions in the search space.

BCDR can be implemented with different search orders, such as best-first, depth-first and branch\&bound, to meet the needs of different applications. We will first give an overview of the BCDR algorithm, and then discuss the conflict learning and resolution in detail. The pseudo code of BCDR is given in Algorithm 1.

BCDR starts with an empty candidate in the queue (Line 1). A candidate, Cand, is a 4-tuple $\left\langle A, R_{e}, C_{r}, C_{\text {cont }}\right\rangle$ with assignments $A$, continuous temporal relaxations $R_{e}$, resolved conflicts $C_{r}$ and continuously resolved conflicts $C_{\text {cont }} \subseteq C_{r}$, all being empty lists in the first candidate. BCDR continues looping until the first relaxation is found that makes the CCTP consistent (Line 11). If BCDR does not find a consistent relaxation until the queue is exhausted, it returns null, indicating that no relaxation exists for the input CCTP (Line 25).

Within each loop, BCDR first dequeues the best partial candidate, Cand (Line 7). It checks if Cand resolves all known conflicts (Line 8). If not, an unresolved conflict currConf will be returned by function UNRESOLVEDCONFLICTS, which compares the resolved conflicts $C_{r}$ in Cand with all known conflicts $C$. currConf is then used for expanding Cand by function ExpandOnCONFLict (Line 22). The child candidates of Cand, which includes both discrete and continuous relaxations for the known conflicts, will then be enqueued. For example, assume that we apply BCDR to the example presented in Figure 2. When expanding a partial candidate $\{A M=B\}$ with conflict $\left\{A M=B, M S=Y, \mathrm{UB}\left(C_{17}\right)-\operatorname{LB}\left(C_{7}\right)\right.$ - $\left.\mathrm{LB}\left(C_{2}\right)-\mathrm{LB}\left(C_{15}\right)-\mathrm{LB}\left(C_{4}\right)-\mathrm{LB}\left(C_{9}\right)\right\}$, BCDR will create three child candidates that extends the partial candidate using the two alternative assignments of $M S, X$ and $Z$ and a temporal relaxation to the upper bound of $C_{17}$ (Figure 12b). All expanded candidates will then be added back to $Q$.

If Cand resolves all known conflicts, BCDR then checks if it is complete, which means that no more variables can be assigned, by comparing its assignments and all unassigned variables in the CCTP (Line 10). If Cand is incomplete, BCDR will expand it using the assignments to one unassigned variable through function EXPANDONVARIABLE (Line 19). 
Input: A CCTP $T=\left\langle P, Q, V, E, R E, L_{p}, L_{e}, f_{p}, f_{e}\right\rangle$.

Output: A relaxation $\left\langle A, R_{e}, C_{r}, C_{\text {cont }}\right\rangle$ that maximizes $f_{p}-f_{e}$.

Initialization:

1 Cand $\leftarrow\left\langle A, R_{e}, C_{r}, C_{\text {cont }}\right\rangle$; the first candidate;

$2 Q \leftarrow\{$ Cand $\}$; a priority queue that records candidates;

$3 C \leftarrow\{\}$; the set of all known conflicts;

4 currConf $\leftarrow\{\}$, newConf $\leftarrow\{\}$; the unresolved conflict that is being expanded on, and the newly discovered conflict. Each is a list of variable assignments and linear expressions;

$5 U \leftarrow V$; the list of unassigned controllable variables;

Algorithm:

$\operatorname{BCDR}(T)$

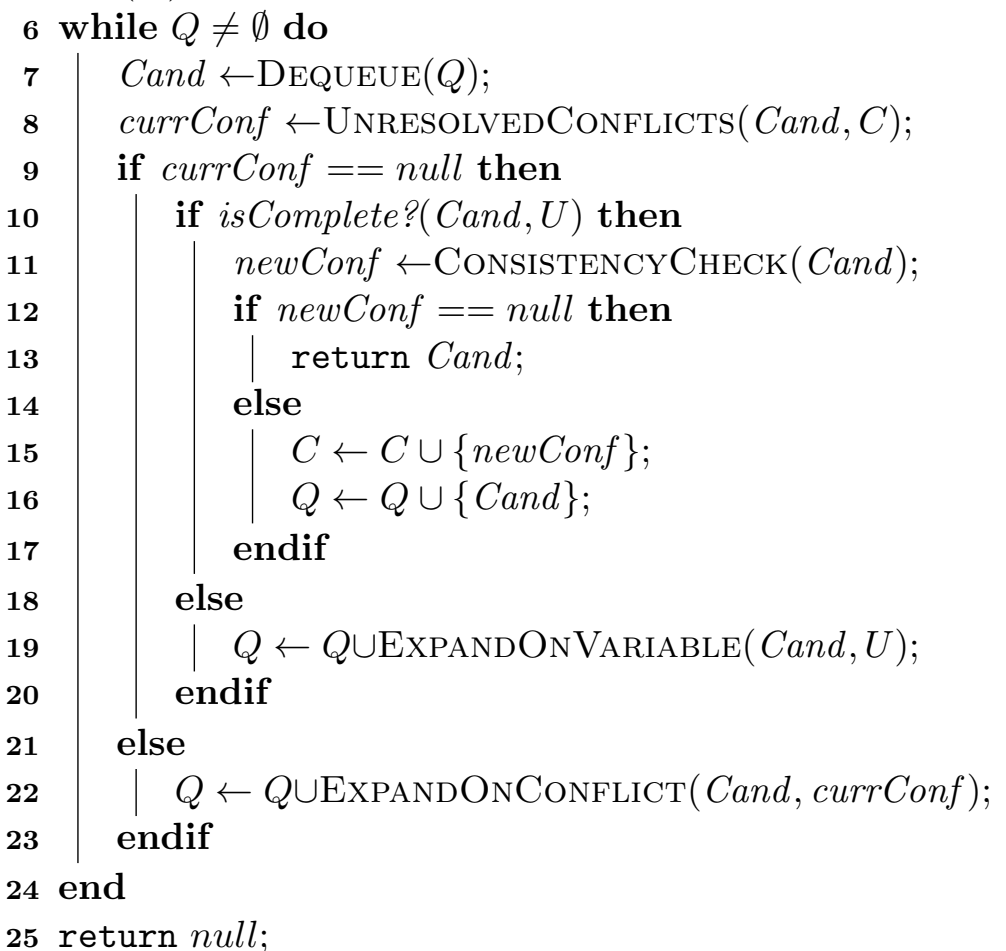

Algorithm 1: The BCDR algorithm

For example, assume again that we need to expand a partial candidate $\{A M=A\}$, but with variable $M S:\{X, Y, Z\}$. This time, we simply create three child candidates that extends the partial candidate using the three possible assignments of $M S$ (Figure 12a).

If $C$ and is complete, BCDR proceeds to check its consistency using function ConsisTENCYCHECK (Line 11). If no conflict is returned, Cand will be returned as the best relaxation for the input CCTP (Line 13). If a new conflict, newConf, is detected by ConsistencyCHECK, BCDR will record it in $C$ and put $C$ and back to the queue for future expansions (Line 15,16), since it now has one unresolved conflict. 


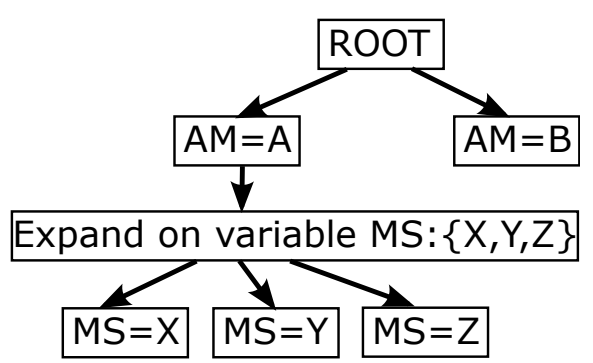

(a)

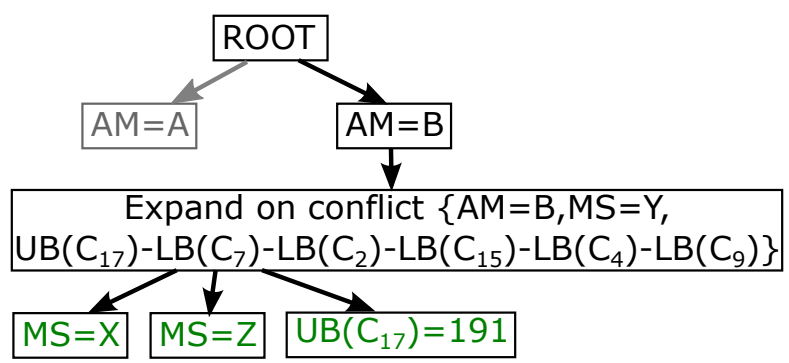

(b)

Figure 12: Examples of expanding on variable and conflict

\subsubsection{Conflict Learning from Consistency Checking}

Conflict learning is the key for resolving over-subscribed temporal plans. They explain the cause of failure and provide guidance for computing necessary relaxations. Previous approaches (Effinger \& Williams, 2005; Li \& Williams, 2005) only extract the set of conflicting episodes and their guard assignments as discrete conflicts.

Definition 13. A discrete conflict is a pair $\langle E$, Guards $\rangle$, where:

- $E$ is a set of conflicting constraints in the CCTP;

- Guards is the set of guard assignments for all constraints in E;

For example, the discrete conflict from the CCTP in Figure 2 can be encoded as the following:

$$
\text { E: } \quad\left\{C_{17}: E-S \in[0,180] ; C_{7}: B_{A^{-}} S \in[30,50] ; C_{2}: B_{L^{-}} B_{A} \in[45,60] ;\right.
$$

Guards: $\quad\{A M=B ; M S=Y\}$

$$
\left.C_{15}: Y_{A}-B_{L} \in[21,25] ; C_{4}: Y_{L}-Y_{A} \geq 65 ; C_{9}: E-Y_{L} \in[30,32]\right\}
$$

While computing continuous temporal relaxations, BCDR needs conflicts of higher resolution, since it tries to resolve the conflict by weakening the temporal bounds to the minimal extent. With the discrete relaxation representation, we can only learn about the constraints that are involved in the conflicts, but not the amount of deviation required for their temporal bounds in order to resolve the conflict. Therefore, we define a new representation of conflicts, called hybrid conflicts, over the temporal bounds in episodes and their guard assignments.

Definition 14. A hybrid conflict is a pair $\langle N C y c l e s$, Guards $\rangle$, where:

- NCycles is a set of linear expressions defined over temporal bounds of constraints, that forms a negative cycle in the equivalent distance graph of the CCTP;

- Guards is the set of guards assignments for all constraints in NCycles;

Each negative cycle in the NCycles set is represented by a linear expression. It represents a necessary constituent of the conflict, and is defined over the lower and upper 
temporal bounds of episodes, with integer coefficients. BCDR learns new conflicts iteratively from grounded CCTPs with different choices made. Given a complete candidate that assigns all active discrete variables, Function ConsistenCyCHECK evaluates the consistency of all activated temporal constraints. The function implements the Bellman-Ford algorithm (Bellman, 1956; Ford, 1956) for checking temporal consistency. If the candidate is temporally inconsistent, the algorithm will return a simple negative cycle as the cause of failure. We can extract the minimal inconsistent set of constraints, also called minimal conflict (Liffiton, Moffitt, Pollack, \& Sakallah, 2005), using this simple negative cycle. For example, Figure 13 shows a simple negative cycle detected in the CCTP presented in Figure 2: the mission time is too tight for both tasks at site $B$ and $Y$.

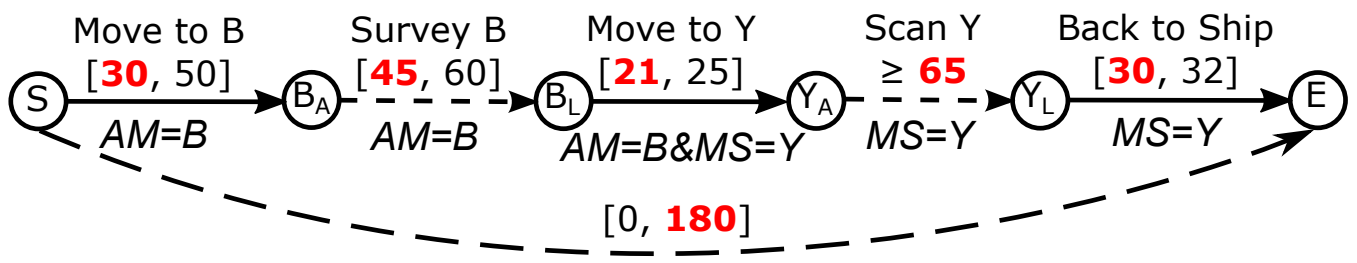

Figure 13: A negative cycle in Rich's mission CCTP (the guard assignments for each constraint are shown below them)

Given the negative cycle, we can encode a hybrid conflict for it as:

$$
\begin{array}{ll}
\text { NCycles: } & \left\{\mathrm{UB}\left(C_{17}\right)-\mathrm{LB}\left(C_{7}\right)-\mathrm{LB}\left(C_{2}\right)-\mathrm{LB}\left(C_{15}\right)-\mathrm{LB}\left(C_{4}\right)-\mathrm{LB}\left(C_{9}\right) ;\right\} \\
\text { Guards: } & \{A M=B ; M S=Y\}
\end{array}
$$

In summary, BCDR learns hybrid conflicts from temporally inconsistent candidates, which are composed of the temporal bounds of constraints involved in negative cycles and the guard assignments of these constraints. Each negative cycle is encoded by a linear expression over the temporal bounds. Therefore, given the original values of these temporal bounds, the value of this expression must evaluate to negative.

\subsubsection{Generalized Conflict Resolutions}

Given a conflict detected in a CCTP, we can compute their resolutions and use them to expand our search tree, such that future expansions of the candidates will not enter the infeasible region represented by this conflict again. This is the core principle behind conflictdirected search. Previous approaches generate discrete relaxations by either flipping the assignments to the discrete variables (Williams \& Ragno, 2002; Bailey \& Stuckey, 2005) or suspending temporal constraints (Moffitt \& Pollack, 2005). BCDR generalizes the conflict resolution to include both discrete and continuous relaxations: the discrete relaxations deactivate constraints in conflicts by flipping their guard assignments, while the continuous relaxations weakens the temporals bounds of them in order to resolve the conflicts.

Given a candidate and a hybrid conflict, Function ExPANDONCONFLICT generates new candidates using the resolutions to the conflict (Algorithm 2). There are two stages in computing the resolutions: First, we generate resolutions for the conflict by negating variable assignments (Line 4-15). If a variable $p_{i}$ is conditioned on other assignments, in addition to flipping the assignment to $p_{i}$, we can also negate its guards. This deactivates the variable 
Input: A candidate to expand $\operatorname{Cand}\left\langle A, R, C_{r}, C_{\text {cont }}\right\rangle$ and an unresolved conflict currConf.

Output: A set of expanded candidates newCands.

Initialization:

1 newCands $\leftarrow\{\}$; collection of newly generated candidates;

2 Confs $\leftarrow C_{\text {cont }} \cup\{$ currConf $\}$; conflicts to be resolved continuously;

$3 A_{\text {alter }} \leftarrow\{\}$; collection of alternative assignments for the ones in currConf;

Algorithm:

EXPANDONCONFLiCT(Cand,currConf)

4 for $a \in \operatorname{currConf}$ do

$5 \quad A_{\text {alter }}=A_{\text {alter }} \cup \operatorname{Get} \operatorname{Altennatives}(a)$;

6 for $a_{l} \in \operatorname{labels}(a)$ do

$7 \quad A_{\text {alter }}=A_{\text {alter }} \cup \operatorname{Get} \operatorname{AlteRnAtives}\left(a_{l}\right)$;

8 end

9 end

10 for $a_{\text {extend }} \in A_{\text {alter }}$ do

11 if notCompeting $\left(A, a_{\text {extend }}\right)$ then

$12 \mid$ Cand $_{\text {new }} \leftarrow\left\langle A \cup\left\{a_{\text {extend }}\right\}, R, C_{r}, C_{\text {cont }}\right\rangle$;

$13 \quad$ newCands $\leftarrow$ new Cands $\cup\left\{\right.$ Cand $\left._{\text {new }}\right\}$;

14 end

15 end

$16 E_{\text {relax }} \leftarrow$ EXtraCtConstraints $($ Confs $)$;

$17 f_{o b j} \leftarrow \sum_{e \in E_{\text {relax }}} f_{e}(\Delta e)$;

$18 R_{\text {new }} \leftarrow$ Optimize $\left(f_{o b j},\left\langle E_{\text {relax }} \geq \mathbf{0}\right\rangle\right)$;

19 if $R_{\text {new }} \neq$ null then

$20 \quad$ Cand $_{\text {new }} \leftarrow\left\langle A, R_{\text {new }}, C_{r}, C_{\text {cont }}\right\rangle$;

$21 \quad$ newCands $\leftarrow$ newCands $\cup\left\{\right.$ Cand $\left._{\text {new }}\right\}$;

22 end

23 return newCands;

Algorithm 2: Function ExpandOnConflict

and resolves the conflict. Assuming that we have to make an additional decision on the imaging method, using either stereo camera or mono camera, for imaging methane seep site Y. Then for a conflict that involves assignment IMAGE $E_{Y}=S T E R E O$, given that we know variable $I M A G E_{Y}$ has label $M S=Y$, we can resolve the conflict by flipping the assignment to either $I M A G E_{Y}$ or $M S: I M A G E_{Y}=M O N O, M S=X$ or $M S=Z$.

In the second stage, we compute the optimal continuous relaxation to the relaxable temporal bounds (Line 16-22), which provides the lower bound on the cost for resolving the conflict continuously. We formulate the relaxation as an optimization problem with linear constraints (Line 16) and linear/quadratic objective function (Line 17). The objective function is the minimization over the sum of the relaxation costs of all relaxable constraints, as in Equation 2. The variables in this optimization problem are $L B_{i}^{\prime} \mathrm{s}$ and $U B_{i}^{\prime} \mathrm{s}$, which are the relaxed bounds for each relaxable temporal constraint. For relaxable requirement constraints, their new temporal bounds must be no tighter than the original bounds, as in 
Equation 3. The resolution constraints in Equation 4 are added to ensure that all known conflicts are repaired by the resolution. Given $m$ conflicts, the same number of resolution constraints will be added, each representing the negation of one linear expression in each conflict (Line 18).

Problem 1 (Conflict resolution).

$$
\begin{array}{ll} 
& \min _{l b_{i}^{\prime}, u b_{i}^{\prime}} \sum_{i=1}^{|R E|} f_{e}\left(l b_{i}^{\prime}\right)+f_{e}\left(u b_{i}^{\prime}\right) ; \\
\text { s.t. } & l b_{i}^{\prime}-l b_{i} \leq 0, \quad u b_{i}^{\prime}-u b_{i} \geq 0 ; \\
& \text { Conflict }_{1} \geq 0 ; \quad \text { Conflict }_{2} \geq 0 ; \ldots \quad \text { Conflict }_{m} \geq 0
\end{array}
$$

For example, the conflict in Figure 13 involves six temporal bounds. Among them, three are relaxable $\left(C_{17}, C_{2}, C_{4}\right)$ that can be relaxed. We can define the following optimization problem for computing the optimal continuous relaxations to resolve this conflict:

$$
\begin{gathered}
\min \left(f_{e}\left(u b_{C 17}^{\prime}\right)+f_{e}\left(l b_{C 2}^{\prime}\right)+f_{e}\left(l b_{C 4}^{\prime}\right)\right) ; \\
\text { s.t. } u b_{C 17}^{\prime}-u b_{C 17} \geq 0 ; l b_{C 2}^{\prime}-l b_{C 2} \leq 0 ; l b_{C 4}^{\prime}-l b_{C 4} ; \leq 0 \\
u b_{C 17}^{\prime}-l b_{C 7}-l b_{C 2}^{\prime}-l b_{C 15}-l b_{C 4}^{\prime}-l b_{C 9} \geq 0 ;
\end{gathered}
$$

The solution to the above optimization problem is a set of relaxed bounds of $C_{17}, C_{2}$, and $C_{4}$ that resolves the conflict while minimizing the cost. In this case, the best relaxation is: set $l b_{C 4}^{\prime}$ to 50 and $u b_{C 17}^{\prime}$ to 185 . The cost is 27.5 . In fact, this problem can also be viewed as a Simple Temporal Problem with Preferences. Khatib et al. (2001) demonstrates that finding the optimal solution to a STPP with semi-convex preferences is tractable. In real world applications, we may substitute different optimization algorithms, depending on the objective functions, to improve efficiency.

In this example, BCDR will generate three constituent relaxations for the hybrid conflict: two new assignments derived from flipping guard assignments, and one set of continuous relaxations for the temporal bounds. They are used to extend the partial candidates so that future extensions of it will not run into the same conflict again, as demonstrated in Figure 12b. When there are multiple unresolved conflicts for a candidate, BCDR is set to expand on the conflict with the least amount of variable assignments first (implemented in function UNRESOLVEDCONFLICTS): this will produce a smaller set of constituent relaxations, and allow a larger portion of the search space to be pruned.

\subsection{BCDR-U: Computing Relaxations that Restore Controllability}

As stated in the beginning of this section, for problems with uncertain durations, such as CCTPUs, the consistency-based approach cannot find relaxation that is robust to all possible outcomes of the uncertainty. Here, we present the extension for conflict learning and resolution procedures that accounts for uncontrollable duration, which allows BCDR to enumerate strongly and dynamically controllable relaxations for CCTPUs. These changes are presented in Algorithm 3. First, function ControllabilityCheCK replaces the consistency check in BCDR presented earlier. Depending on the type of solution required, it 
checks either strong or dynamic controllability, and returns a conflict if the candidate fails the test. The key is to learn conflicts from strong and dynamic controllability checking algorithms, and the conflict could be a mixed set of requirement constraints and uncertain duration. Second, Function ExPANDONCONFLICT is extended to handle conflicts with uncertain durations, whose resolution may involve relaxing requirement constraints and tightening uncertain durations.

Input: A CCTPU $T=\left\langle P, Q, V, V_{r}, E, E_{u}, R E, R E_{u}, L_{p}, L_{e}, f_{p}, f_{e}\right\rangle$.

Output: Assignments and relaxations $\left\langle A, R_{e}, R_{u}, C_{r}, C_{\text {cont }}\right\rangle$ that maximizes $f_{p}-f_{e}$.

Initialization:

1 Cand $\leftarrow\left\langle A, R_{e}, R_{u}, C_{r}, C_{\text {cont }}\right\rangle$; the first candidate;

$2 Q \leftarrow\{C a n d\}$; a priority queue that records candidates;

$3 C \leftarrow\{\}$; the set of all known conflicts;

4 currConf $\leftarrow\{\}$, newConf $\leftarrow\{\}$; the unresolved conflict that is being expanded on, and the newly discovered conflict. Each is a list of variable assignments and linear expressions;

$5 U \leftarrow V$; the list of unassigned controllable variables;

Algorithm:

BCDR-U $(T)$

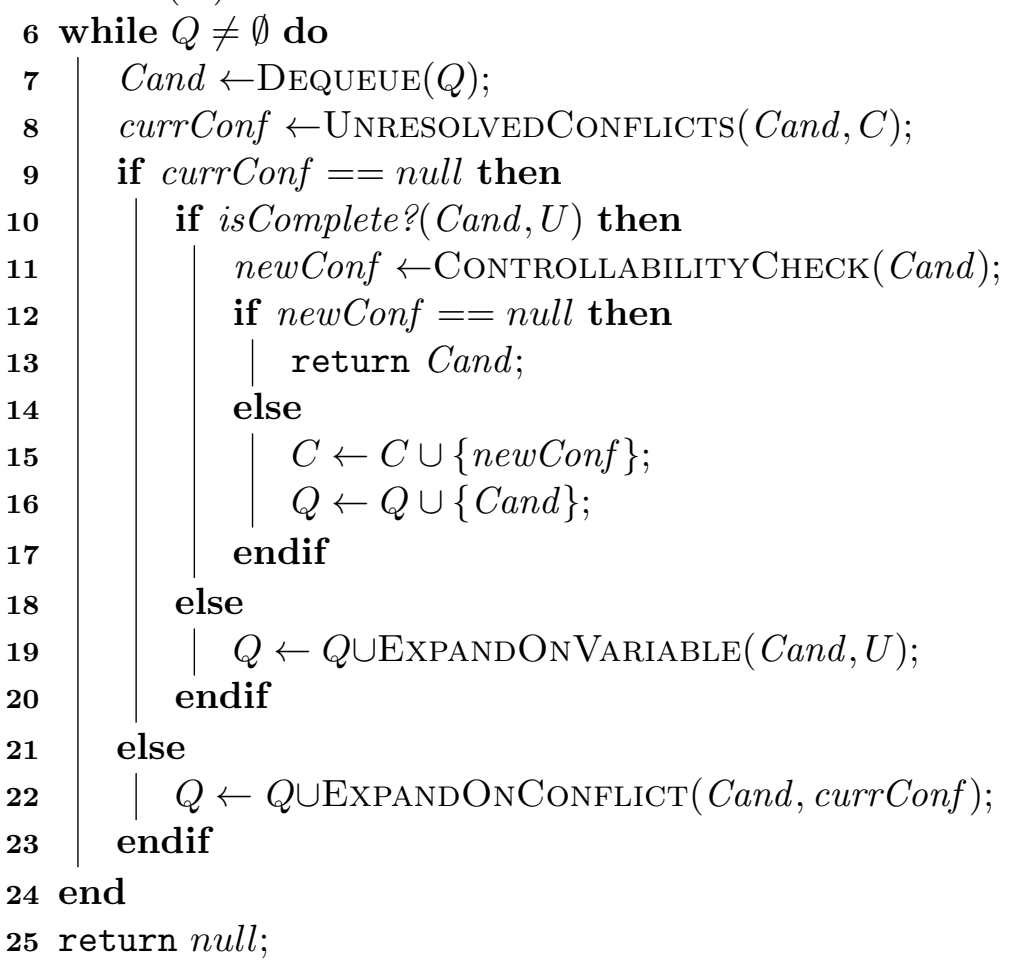

Algorithm 3: BCDR-U for solving CCTPUs

\subsubsection{Conflict Learning for Strong Controllability}

For CCTPs, a conflict is an inconsistent set of temporal bounds from controllable durations.

It can be detected by negative loop detection algorithms: a negative cycle in the equivalent 
distance graph of a grounded CCTP can be mapped to a set of conflicting constraints. This is because of the one-to-one mapping between the distance edges and the lower/upper temporal bounds of constraints. However, this method does not apply to controllability checking algorithms. Due to the reduction procedures in both strong and dynamic controllability checking, the one-to-one mapping property is not preserved: during reductions, new distance edges are created and added to the graph, and the weights of some edges are modified. We cannot extract the sets of conflicting constraints from the negative loops in reduced graphs directly.

The key to solve this issue is to understand what controllable and uncertain duration contributed to each distance edge in the reduced graph. We name these constraints the supporting constraints. The supporting constraints for an edge include the source constraint and the constraints that modify the weight of the edge during reduction. We extend the polynomial time algorithm introduced by Vidal and Fargier (1999) with additional procedures for recording supporting constraints during reductions (Algorithm 4). This extension enables the algorithm to extract a conflict from a negative loop in the reduced graph. The input to it is a grounded CCTPU without any unassigned variables. There are three major steps in this algorithm:

- Map the grounded CCTPU to its equivalent distance graph (Line 1) and record the supporting constraints of each distance edge in the graph with its source, which is either an upper or lower bound of a temporal constraint.

- Reduce all non-contingent edges that start (Line 14) or end (Line 5) at an uncontrollable node using the triangular reduction rules (Figure 14). If constraint A is reduced to $\mathrm{C}$ through $\mathrm{B}$, the supporting constraints of $\mathrm{C}$ will be updated to the union of the supporting constraints of A and B (Line 11, 20).

- After the reductions, we run the Bellman-Ford algorithm on the reduced graph (Line 24). If a negative loop is detected, we collect the supporting constraints of all its edges into a set (Line 25) and return it as a conflict that makes the problem uncontrollable. Otherwise, the function returns null to indicate that the input CCTPU is strongly controllable.

We demonstrate this process using a temporal network with four constraints (Figure 14a): $\mathrm{A}$ and $\mathrm{B}$ are uncertain durations; $\mathrm{C}$ and $\mathrm{D}$ are requirement constraints. First, we map the network to its equivalent distance graph (Figure 14b). Each distance edge in the graph is labeled with its weight and supporting constraints. The subscript after the constraint name, either $\mathrm{U}$ or $\mathrm{L}$, specifies if the distance edge is generated from the upper or lower bound of the constraint.

There are two non-contingent edges in the graph, S2-S1 and E1-E2, and E1-E2 starts and ends at received nodes (denoted by squares in the graph). We first reduce it using edge $\mathrm{S} 2-\mathrm{E} 2$, a contingent edge that shares the same end node with E1-E2. The result is a new edge E1-S2 with weight -2 and supporting constraints $C_{L}, B_{U}$, which are the union of the supporting constraints of E1-E2 and S2-E2 (Figure 14c). Since E1-S2 starts at an received node, we can further reduce it using E1-S1. The result is edge S1-S2 with weight 3 and supporting constraints $C_{L}, B_{U}, A_{L}$ (Figure $14 \mathrm{~d}$ ). 
Input: A grounded CCTPU $T=\left\langle V, E, E_{u}, L_{e}, L_{p}\right\rangle$.

Output: A conflict $\left\langle A, E^{\prime}, \operatorname{Exp}\right\rangle$ that makes $T$ uncontrollable

Algorithm:

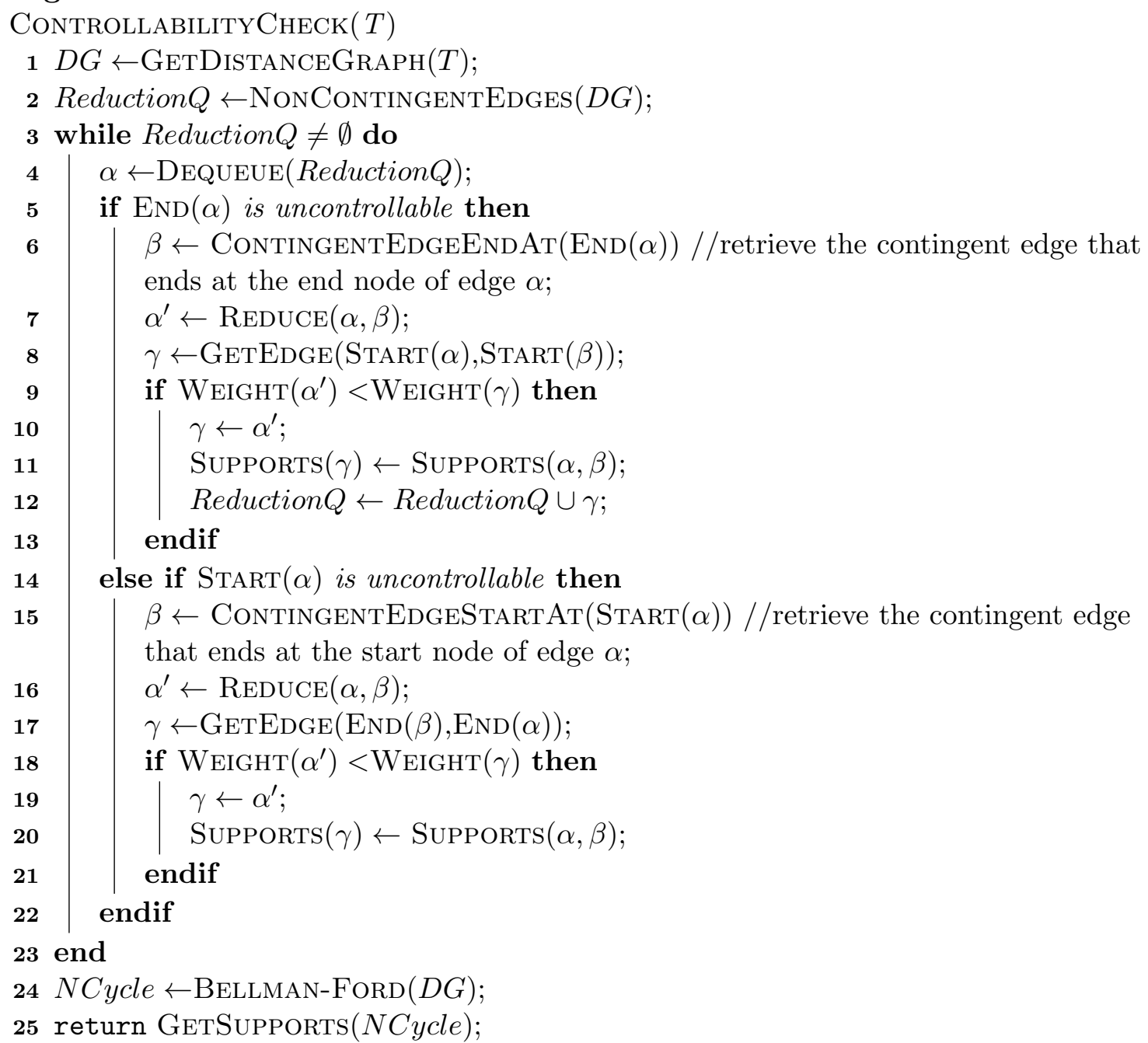

Algorithm 4: Strong controllability checking algorithm

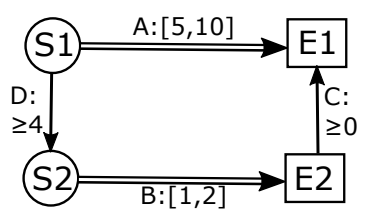

(a)

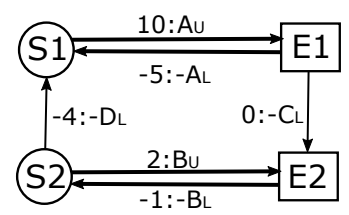

(b)

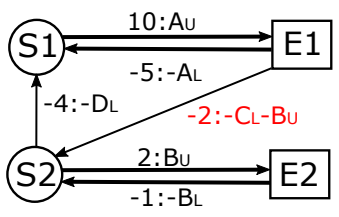

(c)

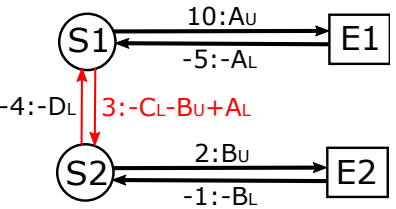

(d)

Figure 14: Supports recording during the triangular reduction for checking strong controllability

It can be seen from the reduced graph that there is a negative cycle of two edges: S1-S2 and S2-S1. The negative cycle indicates that the original STNU is not strongly 
controllable, and the supporting constraints of these two edges, $\left\{A_{L}, B_{U}, C_{L}, D_{L}\right\}$, are in conflict and cause the failure. The linear expression in the NCycles component of this conflict is $-L B(D)-L B(C)-U B(B)+L B(A)$, whose value is -1 without any relaxations to the temporal bounds.

Using this algorithm for checking strong controllability and extracting conflicts does not add much overhead: it takes the same order of magnitude in time compared to consistency checking algorithms. Given a problem with $V$ events and $E$ temporal constraints, there will be at most $2 E$ reductions and support constraint recordings. The time complexity of strong controllability is thus the same order of magnitude as consistency checking: both are dominated by the $O(V E)$ negative cycle detection.

\subsubsection{Conflict Learning for Dynamic Controllability}

Our approach for learning conflicts from dynamic controllability checking algorithm is similar to that for strong controllability. We extend the FASTDCCHECK algorithm, introduced by Morris (2006), with additional steps in its reduction procedures to record the supporting constraints of reduced edges. Its pseudo code is presented in Algorithm 5.

Input: A grounded CCTPU $T=\left\langle V, E, E_{u}, L_{v}, L_{p}\right\rangle$.

Output: A conflict $\left\langle A, E^{\prime}, \operatorname{Exp}\right\rangle$ that makes $T$ uncontrollable

Algorithm:

Controllability Check $(T)$

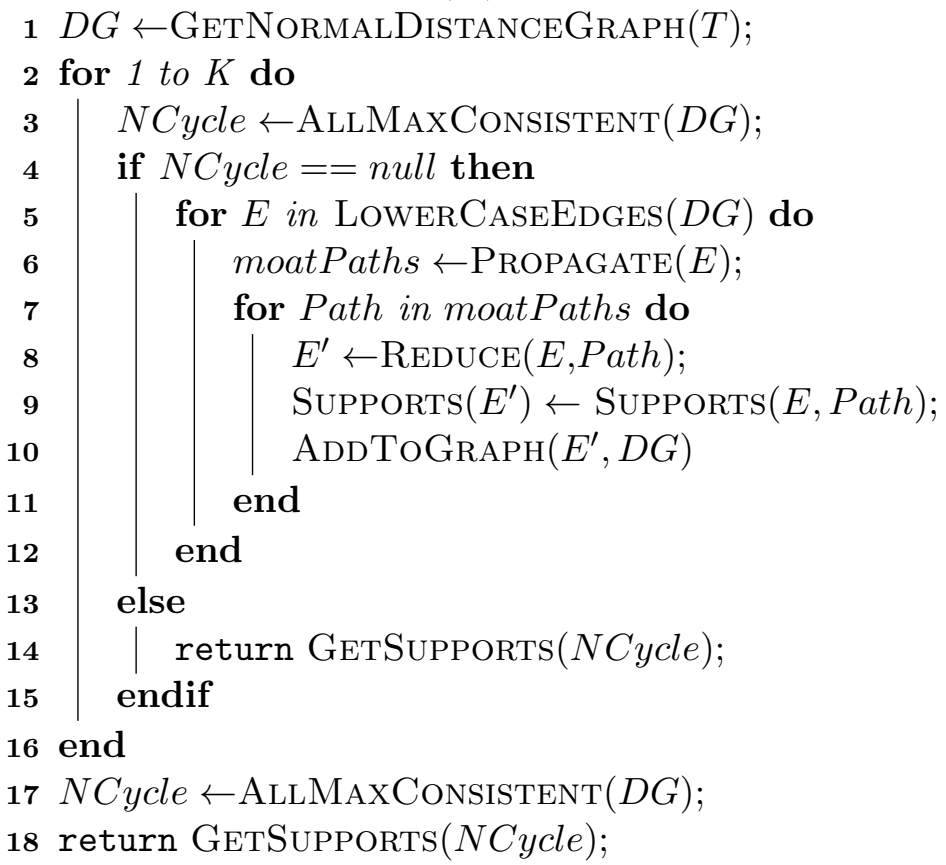

Algorithm 5: Modified FASTDCCHECK algorithm for learning conflicts from uncontrollable networks

As proved by Morris (2006), an STNU is dynamically controllable if and only if it does not have a semi-reducible negative cycle. The FASTDCCHECK algorithm is designed based on this theorem. It converts the STNU to an equivalent distance graph of normal form 
(Line 1) and identifies all negative paths that start with a lower-case edge, called moat paths, through propagations (Line 6). The input STNU is determined to be dynamically controllable if none of these negative paths leads to a semi-reducible negative cycle (Line 3,17 ). The check requires at most $K$ iterations (Line 2 ), where $K$ is the number of lower case edges in the equivalent distance graph of the STNU.

During the reduction of moat paths, we record the supporting constraints for each reduced edge (Line 9). If the AllmaxConsistent function, which implements the BellmanForm algorithm on all non-lower case edges, captures a negative cycle in the reduced graph, it will return a conflict that collects the supporting constraints of all edges in the cycle. There are five types of reductions in this procedure (Morris \& Muscettola, 2005; Morris, 2006), and the support recording process is demonstrated for each of them in Figure 15.

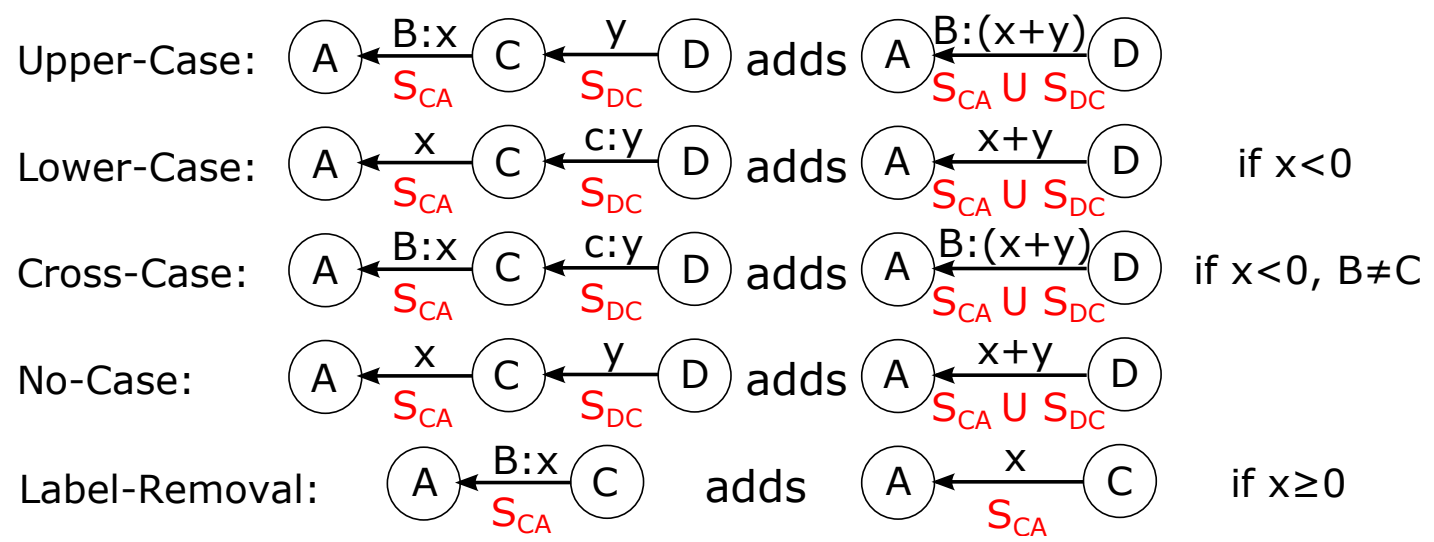

Figure 15: Record supporting constraints during the FASTDCCHECK reductions

Next, we demonstrate the conflict learning process using a simple dynamic controllability checking example (Figure 16). There are three events, E1, E2 and E3, in this example STNU. These events are connected by two constraints A and B: A is an uncertain duration with a bound of $[10,15]$, while $\mathrm{B}$ is a requirement constraints with a bound of $[1,1]$. The first step of controllability checking is to map the STNU to a normalized form (Morris \& Muscettola, 2005), which decouples the lower bounds from each uncertain duration (Figure 17). We can then generate the equivalent distance graph using the normalized STNU. Note that each distance edge in the graph, including conditional edges, is labeled with a linear expression over constraints. The expression encodes the source of an distance edge's weight value, such as the example in Figure 18.

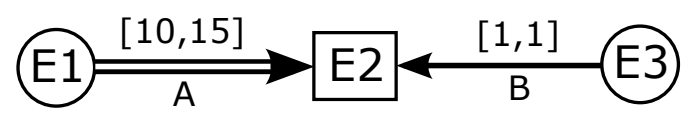

Figure 16: The original STNU

The next step is to identify and reduce all moat paths in the distance graph using the iterative method introduced by Morris (2006). In this example, there is only one valid moat path: $E 1^{\prime} \rightarrow E 2 \rightarrow E 3$. This path has a negative weight, starts with a lower-case edge, and can be reduced to a single edge using a lower-case reduction. The reduced edge (represented 


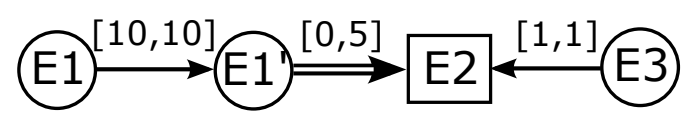

Figure 17: The normalized STNU

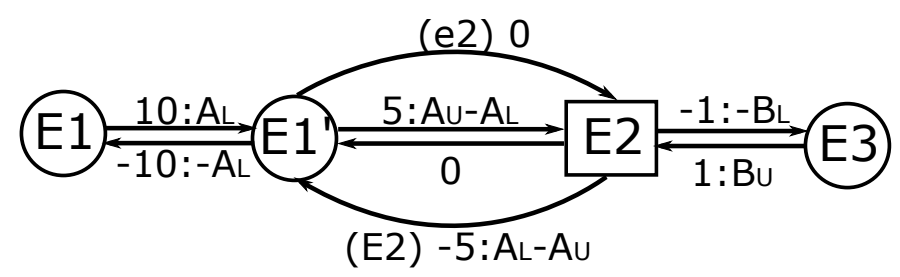

Figure 18: The equivalent distance graph of the STNU

by a dotted arrow in Figure 19) of the moat path has a weight of -1 , and is supported by a linear expression, $-B_{L}$, that combines the expressions of all edges in the moat path.

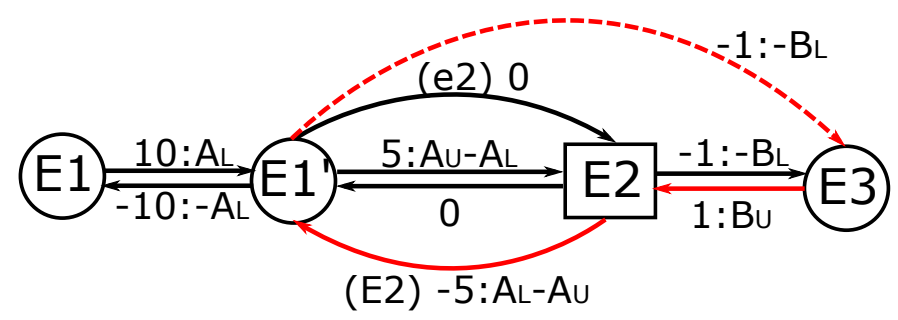

Figure 19: The distance graph with a reduced edge

After all applicable reductions, the final step is to run AllmaxConsistent check on the resulting graph, which checks the consistency of the graph without the lower-case edges. It will reveal any negative cycle in the reduced graph, whose existence indicates that the STNU is not dynamically controllable. In this example, one negative cycle can be detected that contains edge $E 3 \rightarrow E 2, E 2 \rightarrow E 1^{\prime}$, and the reduced edge $E 1^{\prime} \rightarrow E 3$. From this cycle, we can identify the linear expression that caused this conflict from all distance edges in the cycle: $U B(B)+L B(A)-U B(A)-L B(B)$. In addition, there is another subtle but necessary element of this conflict: the reduction that adds edge $E 1^{\prime} \rightarrow E 3$. The negative cycle would not exist without this reduced edge. Therefore, the expression that supports the reduction, $-L B(B)$, which guarantees a negative weight for the moat path, is included in the conflict. The conflict we can extract from the STNU is a conjunction of two linear expressions, $\left\{B_{U}+A_{L}-A_{U}-B_{L} ;-B_{L}\right\}$. Making any one of them non-negative will resolve this conflict.

In summary, learning conflicts from dynamic controllability checking with FASTDCCHECK requires recording the supporting expression for each distance edge and reduction. Once a negative cycle is detected, we can extract a conflict by collecting (1) the expressions for each edge in the cycle; and (2) the expressions required by the reductions that added edges to the cycle. The conflict is a conjunction of these linear expressions, which are all negative and defined over the temporal bounds of constraints. 
Currently, extracting conflicts from dynamic controllability checking is significantly harder than that for strong controllability, even though the extra time and space required by recording supports during reductions does not increase the overall complexity of the algorithm. The FASTDCCHECK algorithm is currently the second fastest DC checking algorithm with a complexity of $O\left(N^{4}\right)$, which is an order of magnitude higher than checking strong controllability. To improve run-time efficiency, the algorithm can be terminated and return true after a no-reduction iteration. This is similar to the implementation presented by Morris and Muscettola (2005), and will not affect the correctness of the results. The integration of BCDR with a cubic DC checking algorithm introduced by Morris (2014), is expected to further improve performance and is part of our future work to explore.

\subsubsection{Resolving Conflicts with Uncontrollable Durations}

As described in the beginning of this subsection, both BCDR-U(SC) and BCDR-U(DC) algorithms use the resolutions to unresolved conflicts to expand the search tree. For CCTPUs, there are three options for resolving conflicts, which may include both controllable constraints and uncertain duration:

- Flipping the guard assignments to deactivate constraints.

- Relax the temporal bounds of requirement constraints.

- Tighten the temporal bounds of uncertain durations.

The conflict resolution process for CCTPUs, similar to the one for CCTPs, is separated into two stages. The first stage is identical to that for CCTPs: we look for alternative assignments that can deactivate one or more constraints in the conflict, and use them to generate new candidates.

The second stage implements option 2 and 3. We compute the continuous relaxations to the temporal bounds in the conflicts. The linear expressions in each conflict's negative cycles provide guidance for EXPANDONCONFLICT to resolve over-constrained problems. A conflict is eliminated if any of its linear expressions is made non-negative. For example, we can resolve the conflict in Figure 19 using the following two approaches:

- Set $B_{U}+A_{L}-A_{U}-B_{L} \geq 0$, e.g. increasing $A_{L}$ to 15 .

- Set $-B_{L} \geq 0$, e.g. lowering $B_{L}$ to 0 .

Intuitively, to resolve a conflict we can directly require the weight of a previously negative cycle to be non-negative, or we can make sure the reduction that adds an edge never occurs. This choice in conflict resolution is unique to dynamic controllability conflicts: a hybrid conflict from consistency or strong controllability checking only introduces one linear expression. This choice provides more flexibility in conflict resolution, although it also increases the complexity of the problem: to compute the optimal resolutions, BCDR$\mathrm{U}(\mathrm{DC})$ may need to evaluate all possible repairs for all conflicts. The search branches each time BCDR-U(DC) expands on a conflict. If a quick response is desired by the user, BCDR-U(DC) should be implemented with an anytime search strategy. 
Once an expression is selected for each conflict, we can again formulate a constraint optimization problem and compute the resolutions using an optimization solver in polynomial time, assuming that the objective function remains semi-convex. There are two categories of variables in the optimization problem: relaxed lower and upper bounds for requirement constraints $\left(l b_{i}^{\prime}\right.$ and $\left.u b_{i}^{\prime}\right)$ and tightened lower and upper bounds for uncertain durations $\left(l b_{u j}^{\prime}\right.$ and $u b_{u j}^{\prime}$ ). These are given in Problem 2.

Problem 2 (Conflict resolution with set-bounded uncertain durations).

$$
\begin{array}{ll} 
& \min _{l b_{i}^{\prime}, u b_{i}^{\prime}, l b_{u j}^{\prime}, u b_{u j}^{\prime}} \sum_{i=1}^{\left|R E \backslash R E_{u}\right|} f_{e}\left(l b_{i}^{\prime}\right)+f_{e}\left(u b_{i}^{\prime}\right)+\sum_{j=1}^{\left|R E_{u}\right|} f_{e}\left(l b_{u j}^{\prime}\right)+f_{e}\left(u b_{u j}^{\prime}\right) ; \\
\text { s.t. } & l b_{i}^{\prime}-l b_{i} \leq 0, \quad u b_{i}^{\prime}-u b_{i} \geq 0 ; \\
& l b_{u j}^{\prime}-l b_{u j} \geq 0, \quad u b_{u j}^{\prime}-u b_{u j} \leq 0, \quad u b_{u j}^{\prime}-l b_{u j}^{\prime} \geq 0 ; \\
& \text { Conflict }_{1} \geq 0 ; \quad \text { Conflict }_{2} \geq 0 ; \ldots \quad \text { Conflict }_{m} \geq 0 ;
\end{array}
$$

The constraints in the optimization problem enforce the necessary properties. For CCTPUs, we have an additional set of constraints: for lower and upper bound variables of uncertain durations, their values must be within the range defined by the original bounds, and the new lower bound is smaller than or equal to the new upper bound, encoded by (7). For example, given the conflict in Figure 16, the relaxed bounds for uncertain duration $A, l b_{u A}^{\prime}$ and $u b_{u A}^{\prime}$, must follow $10 \leq l b_{u A}^{\prime} \leq u b_{u A}^{\prime} \leq 15$.

\subsection{BCDR-C: Computing Chance-Constrained Relaxations}

Finally, we present the extension to the BCDR algorithm, called BCDR-C, that allows it to resolve over-constrained cc-pCCTPs. The extension leverages ideas presented by Fang et al. (2014) for grounding probabilistic Simple Temporal Problems (pSTPs) into deterministic STNUs, and uses the conflict-directed framework for efficient conflict detection and resolution. Given a cc-pCCTP, BCDR-C enumerates feasible solutions in best-first order: a solution is a complete set of assignments and a collection of relaxations for temporal bounds and chance constraint. Each resolution supports a grounded STNU whose probability of failure is bounded by the relaxed chance constraint. This requires the conflict-directed approach to support both relaxation and risk allocation: given the grounded STNU of a cc-pCCTP that represents a specific set of choices and risk allocation, BCDR-C will identify the conflicts between constraints and use their resolutions to guide the search towards feasible risk allocation and constraint relaxation. The two key modifications from BCDR-U to BCDR-C are the following:

- First, an additional step of risk-allocation is required for grounding the probabilistic input problem to a STNU. This allows us to check the feasibility and extract conflicts between constraints using the algorithms developed for STNUs.

- Second, in addition to flipping assignments and relaxing temporal bounds, the conflict resolution step can also adjust risk allocation over uncertain durations in order to 
resolve all known conflicts while maintaining the risk taken. Note that this step may require a non-linear optimization solver if the probabilistic distribution of any uncertain duration is non-linear.

Input: A cc-pCCTP $T=\left\langle P, Q, V, V_{r}, E, E_{d}, R E, L_{e}, L_{p}, f_{p}, f_{e}, \Delta_{t}, r \Delta_{t}, f_{\Delta}\right\rangle$.

Output: A solution $\left\langle A, R_{e}, \Delta_{t}^{\prime}, N_{\text {alloc }}, C_{r}, C_{\text {cont }}\right\rangle$ that maximize $f_{p}-f_{e}-f_{\Delta}$.

Initialization:

1 Cand $\leftarrow\left\langle A=\emptyset, R_{e}=\emptyset, \Delta_{t}, N_{\text {default }}, C_{r}=\emptyset, C_{\text {cont }}=\emptyset\right\rangle$; the first candidate with the default risk allocation, and empty sets for assignments, relaxations and continuously resolved conflicts;

$2 Q \leftarrow\{$ Cand $\}$; a priority queue of candidates;

$3 C \leftarrow\{\}$; the set of all known conflicts;

4 currConf $\leftarrow\{\}$, newConf $\leftarrow\{\}$; the unresolved conflict that is being expanded on, and the newly discovered conflict. Each is a list of variable assignments and linear expressions;

$5 U \leftarrow V$; the list of unassigned controllable variables;

\section{Algorithm:}

BCDR-C $(T)$

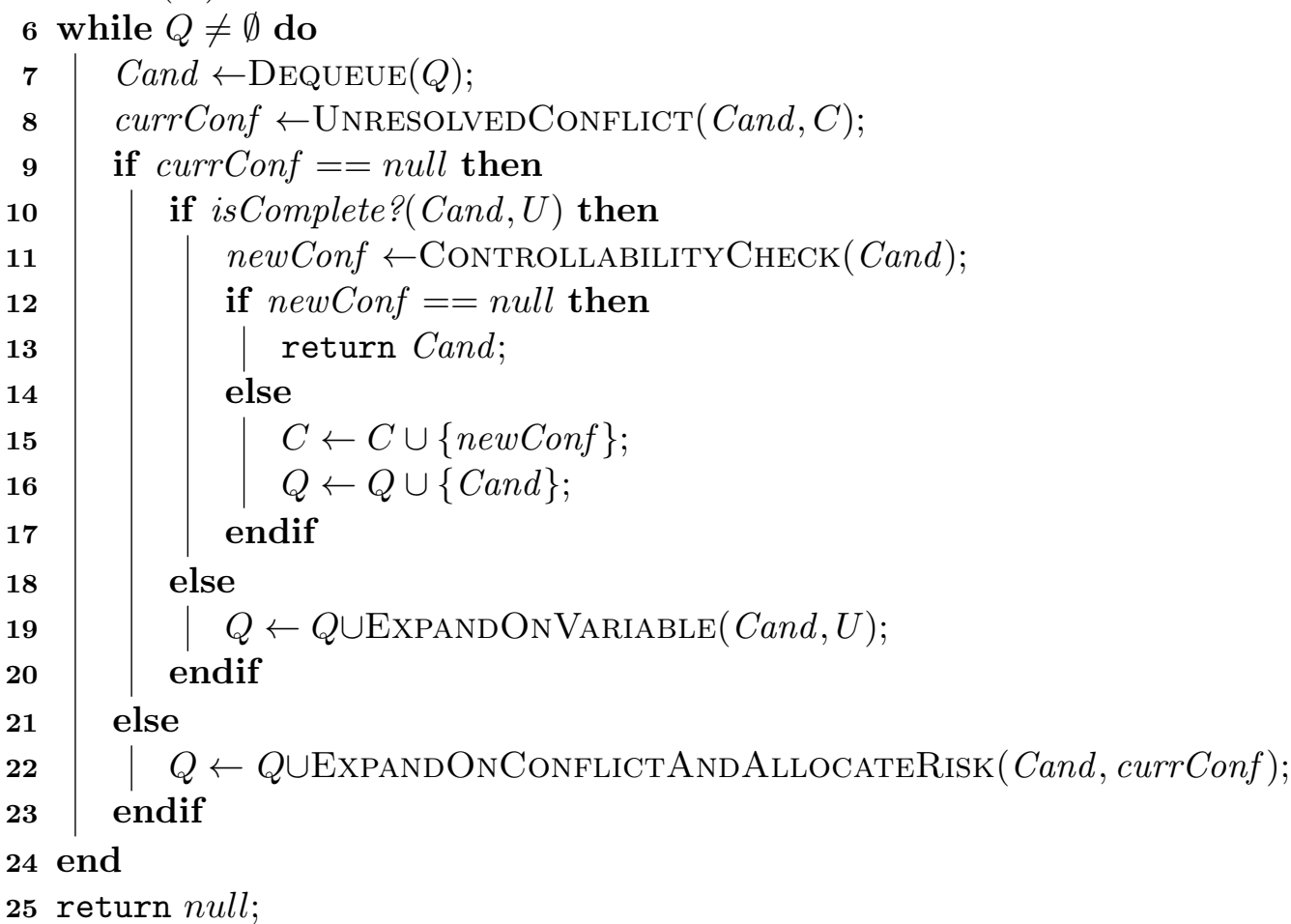

Algorithm 6: BCDR-C algorithm for resolving cc-pCCTPs

We first present an overview of the algorithm that highlights the modifications, then discuss the risk-allocation and chance constraint relaxation procedures in detail. The pseudo code of the chance-constrained version of BCDR-C is presented in Algorithm 6. Similar to the previous two versions, we implement BCDR-C with a priority queue for enumerating 
resolutions in best-first order. The algorithm starts with an empty candidate (Line 1) that has no assignments or relaxations over temporal constraints $\left(A\right.$ and $\left.R_{e}\right)$ and chance constraint $\left(\Delta_{t}\right)$, and empty sets of resolved conflicts $\left(C_{r}\right)$ and continuously resolved conflicts $\left(C_{\text {cont }}\right)$. The candidate is associated with a default risk allocation over all probabilistic uncertain durations, which is represented by a CCTPU $\left(N_{\text {default }}\right)$. The initial allocation is computed from a non-linear solver and is conservative enough to satisfy the chance constraint. For example, we can compute the initial allocation by solving Problem 3 without any linear constraints from conflicts. The initial candidate is the only element in the queue before search starts (Line 2).

Within the main loop, BCDR-C first dequeues the best candidate (Line 7) and checks if it resolves all known conflicts (Line 8). If not, a conflict currConf will be returned by function UnresolvedConflict. The unresolved conflict is then used for expanding Cand (Line 22). All child candidates returned by function ExpandOnConflictAndAllocateRisk resolve currConf while satisfying the chance constraints. The function also computes the risk allocation over probabilistic temporal durations for each candidate, which is added back to the queue for future evaluation and expansion.

If Cand resolves all known conflicts, BCDR-C will proceed to check the controllability of its grounded CCTPU (function ControllabilityCHECK, Line 11). BCDR-C(SC) implements this function with a strong controllability checking algorithm, while BCDR-C(DC)'s function implements dynamic controllability checking. If the grounded network passes the check, Cand will be returned as the best resolution to the cc-pCCTP (Line 13). Otherwise, a new conflict will be returned by this function and recorded for expanding candidates (Line 15). Cand will also be added back to $Q$ since it now has an unresolved conflict (Line 16).

Similar to BCDR and BCDR-U, every solution returned by BCDR-C is valid in that they have a feasible risk-allocation and pass the strong or dynamic controllability check, hence it is easy to prove the algorithm's soundness. On the other hand, unlike BCDR and BCDR-U, BCDR-C is not a complete algorithm in that it may fail to return a solution for some cc-pCCTPs that do have feasible relaxations. This is a result of its conservative risk allocation procedure. We take a union bound approach when calculating the total risk taken across the temporal bounds allocated for all probabilistic durations. It guarantees that the solution returned will operate within the specified risk-bound. However, the conservation causes BCDR-C to overestimate the risk taken. As a result, it may not be able to find a feasible risk allocation for problems with tight risk-bounds, even if one may exist. We will discuss more details on this issue in the following section.

\subsubsection{Risk Allocation and Constraint Relaxation}

Conflicts provide guidance for BCDR-C to resolve over-constrained problems. Given a set of conflicts, we formulate a constrained optimization problem and compute the resolutions using a non-linear optimization solver. To resolve a conflict we can require the weight of any of its linear expressions to be non-negative. There are three categories of variables in the optimization problem: relaxations for temporal constraints $\left(l b_{i}^{\prime}\right.$ and $\left.u b_{i}^{\prime}\right)$, relaxations for chance constraint $\left(\Delta_{t}^{\prime}\right)$, and the allocation of lower and upper bounds for probabilistic durations $\left(l b_{p j}^{\prime}\right.$ and $\left.u b_{p j}^{\prime}\right)$. Each category of variables represents a type of conflict resolution: 
re-allocating risk over probabilistic durations, relaxing the chance constraint, and relaxing temporal constraints. These are given in Problem 3.

Problem 3 (Conflict resolution with chance constraints and probabilistic durations).

$$
\begin{array}{ll} 
& \min _{\Delta_{t}^{\prime}, l b_{i}^{\prime}, u b_{i}^{\prime}} f_{\Delta}\left(\Delta_{t}^{\prime}-\Delta_{t}\right)+\sum_{i=1}^{|R E|} f_{e}\left(l b_{i}^{\prime}\right)+f_{e}\left(u b_{i}^{\prime}\right) ; \\
\text { s.t. } & l b_{p j}^{\prime}-u b_{p j}^{\prime}<0 \\
& l b_{i}^{\prime}-l b_{i} \leq 0, \quad u b_{i}^{\prime}-u b_{i} \geq 0 ; \\
& \text { Conflict }_{1} \geq 0 ; \quad \text { Conflict }_{2} \geq 0 ; \ldots \quad \text { Conflict }_{m} \geq 0 ; \\
& \sum_{r_{j} \in R_{d}} \operatorname{RisK}\left(l b_{p j}^{\prime}, u b_{p j}^{\prime}\right) \leq \Delta_{t}^{\prime}, \quad \Delta_{t}^{\prime} \in\left[\Delta_{t}, 1\right)
\end{array}
$$

The constraints in the optimization problem enforce the necessary properties. For lower and upper bound variables of probabilistic durations, their value can be assigned as long as the lower bound is smaller than the upper bound, encoded by (10). For relaxable requirement constraints, their new temporal bounds must be no tighter than the original bounds, as in (11). For requirement constraints that are not relaxable, their temporal bounds remain unchanged (omitted from the encoding).

The resolution constraints in (12) are added to ensure that all known conflicts are repaired by the resolution, similar to those in Problems 1 and 2. Given $m$ conflicts, the same number of resolution constraints will be added, each representing one linear expression in each conflict. Finally, we add a risk allocation constraint to ensure that the risk taken meets the chance constraint. This constraint is defined over the lower and upper bound variables of all probabilistic durations. Given distributions of each probabilistic duration and the uncertainty bounds chosen, the RISK function computes the probability mass of the regions outside the uncertainty bounds. BCDR-C uses the union bound to upper-bound the total risk taken across all uncertain durations, as this does not rely on assumptions of independence. If the chance constraint is relaxable, we further require that the relaxed chance constraint is lower bounded by the original chance constraint, and upper bounded by 1 . This gives us the flexibility to make trade-offs between risk and performance, if no solution can be found that resolves all conflicts while meeting the current chance constraint. These are described by (13).

The objective function, given in (9), is defined over $f_{\Delta}$ and $f_{e}$ for minimizing the cost of temporal and chance constraint relaxations. In the optimization problem, all domain and conflict resolution constraints are linear, while the chance constraint may be non-linear depending on the probabilistic distributions. BCDR-C uses the SNOPT optimization package (Wachter \& Biegler, 2006) to solve Problem 3 and compute optimal constraint relaxations and risk allocations. If a solution is returned by SNOPT, function EXPANDONCONFLICT will construct a new candidate with its relaxations for temporal and chance constraints. This candidate will then be added as a new branch to BCDR-C's search tree, similar to the process in Algorithms 1 and 3.

Finally, we would like to mention one limitation of BCDR-C on resolving cc-pCCTPs. In many real-world scenarios, people may want to impose different chance constraints over 
different subsets of uncertain durations. The current implementation of BCDR-C, especially the chance-constrained relaxation procedure, only supports a single chance constraint. It is unable to impose different risk bounds on different sets of constraints while computing new relaxations and risk allocations. Constraints covered by different risk bounds may appear in the same conflict, and it is not clear how to distribute the risk to multiple chance constraints during conflict resolution. The solutions to these issues are part of our future work to explore.

\subsection{Incorporating User Inputs as Hybrid Conflicts}

As demonstrated in Section 2, the user can add additional inputs given an unsatisfying solution, or requirements he/she forgot to encode in the original problem. BCDR can incorporate them into its search process as a hybrid conflict, such that all future candidate solutions will respect them. In total, given a solution, three types of inputs can be accepted by BCDR:

- Rejection of an assignment, such as "I do not want to visit the methane seep site X".

- Rejection of a continuous temporal relaxation, such as "The mission duration must be within 4 hours".

- Rejection of a chance constraint relaxation, such as "I cannot take more than $5 \%$ risk of violating any constraints".

BCDR utilizes the conflict-directed approach to efficiently adapt to these inputs. Instead of modifying the input problem and restarting the search process from the beginning, it will record the input as a new conflict and add it to the known conflicts list. The above three types of inputs will be recorded as the following conflicts:

- Rejection of an assignment $X=a$ : a new conflict $X=a$ will be created and added to BCDR's conflict collection, such that it will not appear again in any future solutions.

- Rejection of a temporal relaxation $l b_{i}^{\prime}=a$ or $u b_{i}^{\prime}=b$ : a new hybrid conflict with linear expression $l b_{i}^{\prime}-a \geq 0$ or $b-u b_{i}^{\prime} \geq 0$ will be added to BCDR's conflict collection, such that all future relaxations for $l b_{i}^{\prime}$ or $u b_{i}^{\prime}$ will be bounded by $a$ and $b$, respectively.

- Rejection of a chance constraint relaxation, $\Delta_{t}^{\prime}=a$ : update the domain of variable to $\Delta_{t}^{\prime} \in[0, a]$. There is no need to generate new conflict since the chance constraint applied to all candidate solutions, and we allow one and only one chance constraint in each problem.

The pseudo code of this implementation, called REACTIVEBCDR, is presented in Algorithm 7. Note that the BCDR algorithm presented in earlier sections is wrapped inside the function BCDR. REACTIVEBCDR starts with querying BCDR for a solution to the given temporal problem, either a CCTP, a CCTPU or a cc-pCCTP (Line 4). If no solution can be found to the problem, the algorithm will signal failure and quit (Line 6). Otherwise, the first solution will be presented to the user. If the user accepts it, REACTIVEBCDR will return the solution and terminate (Line 9). If the user rejects it, it will prompt the user for 
Input: T: A CCTP, CCTPU or cc-pCCTP.

Output: Sol: A valid solution, or null if none exists for the input problem.

Initialization:

$1 C \leftarrow\{\}$; the set of all known conflicts kept by BCDR;

$2 Q \leftarrow\{\}$; the priority queue for candidate relaxations kept by BCDR;

Algorithm:

$\operatorname{ReACTIVEBCDR}(T)$

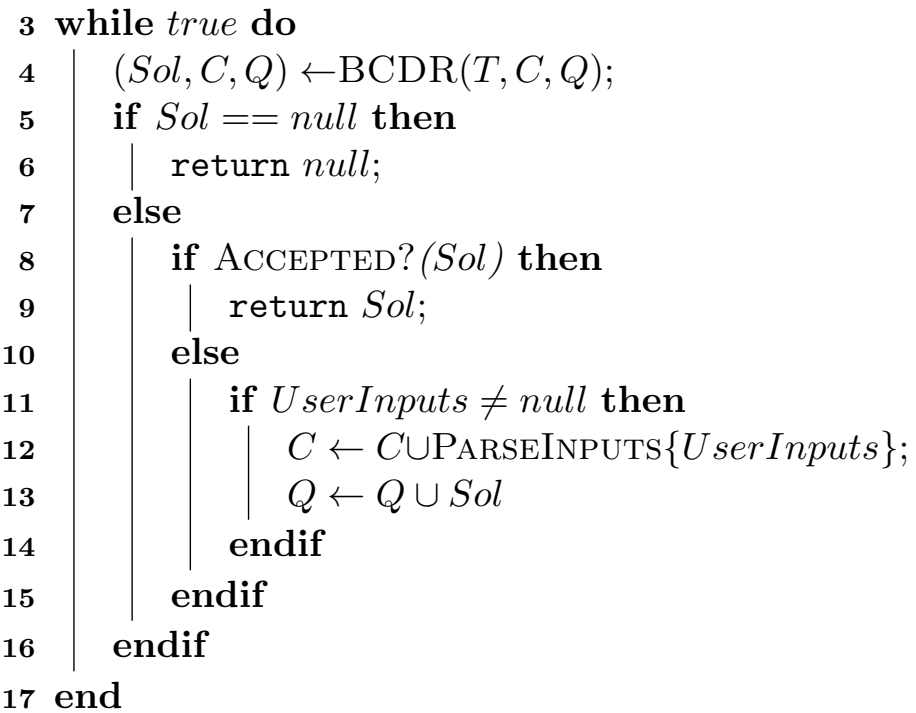

Algorithm 7: The Reactive BCDR algorithm

additional inputs, and encode them into conflicts using the three rules (Line 12). Note that the current solution will also be put back to the queue, too, since it now has an unresolved conflict. If no input is provided, BCDR will move on to find the next best solution, and the current solution is discarded.

\subsection{Implementation Issues and Suggestions}

Finally, we discuss two issues revealed during our experiments with BCDR, and present our solutions to them that will improve the robustness and run-time performance of BCDR for similar types of temporal problems. The first issue is a numerical instability problem that may cause BCDR to become stuck on a certain conflict: the relaxation generator thinks a conflict has been resolved, while the consistency or controllability checker disagrees and keeps returning the same conflict. Our solution is a parameterized negative cycle detection function whose sensitivity can be lowered to match that of the relaxation generator.

The second issue is that the default conflict resolution procedure may be very inefficient for highly over-constrained problems (problems with a large number of conflicts). As the number of conflicts to resolve increases, the conflict relaxation procedure slows down due to the increasing number of constraints to satisfy. However, much of the computation is not very useful, since the relaxations from a previous iteration become useless when a new conflict is discovered: we have to execute the expensive optimization procedure again with more constraints. Our solution is a mixed greedy-optimal relaxation procedure that 
uses discrete relaxation when more conflicts are likely to be discovered, and only runs the continuous relaxation procedure if it is likely that no more conflicts may be discovered.

These issues and resolutions may be of particular interest to readers who are applying BCDR to real-world problems with a large set of constraints and highly connected structure. Note that our experimental results of BCDR will be discussed in the following section: here we focus on the source of these issues and the rationale behind the modification to get BCDR working properly.

\subsubsection{Numerical Issues in Continuous Relaxation}

The conflict-directed framework used by BCDR and all its extensions follow a generate and test approach, which coordinates the generator (for computing continuous relaxations) and the tester (for checking temporal feasibility) to 'challenge' each other until a relaxation that resolves every conflict is found. When the checker discovers a conflict $c$, it requires the linear expression $c_{e}$ of the conflict to be made non-negative. In order to minimize the cost function $f_{e}$, the continuous relaxations generated by the optimizer often makes $c_{e}=0$. However, in some rare cases, after the arithmetic for the reduction process of checking controllability, the value of $c_{e}$ becomes $0-\epsilon$, where $\epsilon$ is a very small number. This causes the checker to re-discover the same conflict: the relaxation generator believes that the conflict can be resolved, hence it will not signal failure and tell BCDR to terminate; while its relaxation never satisfies the checker, which causes BCDR to become stuck.

The problem was observed when BCDR-U(DC) ran into an infinite loop. The number of ExPANDONCONFLICT operation counts kept growing as if there is an infinite set of conflicts to resolve. A further investigation into this issue revealed that in these nonterminating scenarios, the conflicts learned by the controllability checker beyond a certain point are all identical. The continuous relaxation generated by the ExPANDONCONFLICT procedure does not resolve the new conflict, causing the checker to re-discover it again and again. This problem is more often observed on problems with highly connected constraints, which require a large amount of reduction during DC checking and increases the chance of numerical precision issues. Here we do not require the input constraint bounds to be integer or rational values, which will be discussed later in this section as possible resolutions.

We use the example from Figure 19 to demonstrate this problem. Recall that the conflict extracted from dynamic controllability checker for this CCTPU has two linear expressions: $\left\{B_{U}+A_{L}-A_{U}-B_{L} ;-B_{L}\right\}$. Assume that the relaxation generator decided to increase $A_{L}$ to 15.0 , which effectively eliminates the uncertainty in it, we will get the relaxed problem in Figure 20. Next, the problem with the relaxation is passed back to the controllability checker for verification. The checker executes the same reduction procedures shown in Figure 15, and gets a reduced network (Figure 21). However, during the reduction, some of the arithmetic operations may introduce errors and the resulting edge in the network has the incorrect weight of -0.0000000001 , instead of 0 . As can be seen from the graph, the same negative cycle of Edge $E_{3} \rightarrow E_{2}, E_{2} \rightarrow E_{1}^{\prime}$ and $E_{1}^{\prime} \rightarrow E_{3}$ will be detected by the checker, and hence the same conflict will be returned by it, which puts BCDR-U(DC) into an infinite battle with an already resolved conflict.

There are several options to resolve or reduce the chance of running into this numerical issue: we may slightly relax the sensitivity of the controllability checker, set the continuous 


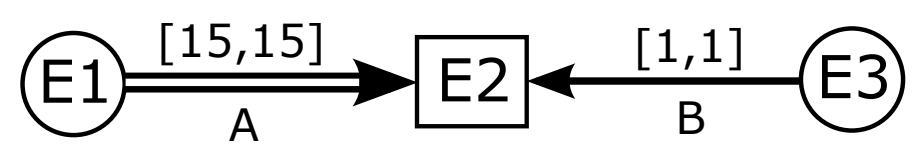

Figure 20: The CCTPU with a relaxed lower bound for uncertain duration $A$

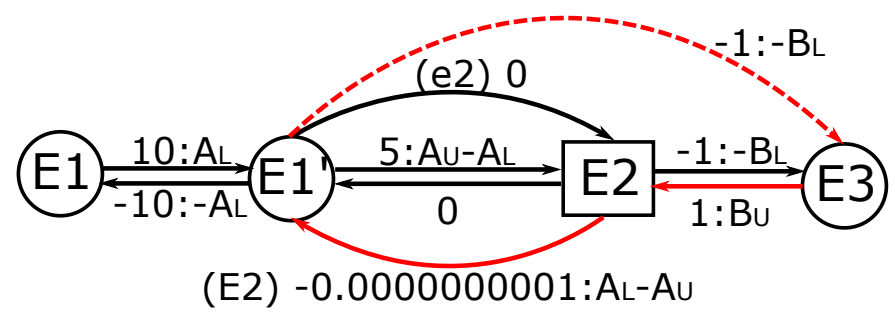

Figure 21: The reduced graph with additional edges

relaxation generator to over-relax a bit, or switch to rational numbers which eliminates the numerical issue completely. We took the first approach since it provides the most intuitive configuration and the flexibility to work in all situations: the over-relaxation approach does not apply to problems whose solution space is very small, such as the RCPSPs used by Cui et al. (2015) in their experiments; the rational number approach requires an approximation step to scale up the temporal bounds and rounding, whose impact on the correctness of BCDR is difficult to estimate. As mentioned in earlier sections, all temporal feasibility checking functions (consistency, strong controllability and dynamic controllability) depend on a negative cycle detection function implemented based on the Bellman-Ford algorithm, and the key in our approach is to loosen the criteria for a negative loop. Therefore, we modified the condition for distance updates in Bellman-Ford, and the changes are highlighted in Algorithm 8.

There are three steps in the Bellman-Ford algorithm: initialization of distance and predecessor for vertices, updating the shortest distances to each vertex from source, and detecting and extracting any negative cycles. The key modification we made is the introduction of a non-negative sensitivity parameter $\epsilon$, which is used during the updates of vertex distances (Line 7) and the extraction of negative cycle (Line 13). It requires the new distance to be $\epsilon$-less than the original distance, instead of just being smaller, effectively making it more difficult for updates to take place. As a result, no negative cycle with value larger than $-\epsilon$ in the original graph will be detected, since such small differences will not be captured during the distance updates. This parameter can be tuned for different applications to eliminate the possibility of numerical issues while maintaining a good precision and reliability. In our experiments, we set $\epsilon$ to $10^{-9}$ and found it to be sufficient to completely eliminate the numerical issues.

\subsubsection{Delayed Conflict Resolutions}

Computing continuous relaxations is the most expensive procedure in BCDR. On the other hand, most of the relaxation computation is not directly contributing to the final solution: we compute relaxations to all known conflicts so that a new candidate can be generated to 


\section{Input:}

$G:\langle V, E, s\rangle$, a weighted directed graph with vertices $V$, edges $E$ and source $s$;

$\epsilon$ : the sensitivity settings for negative cycle detection.

Output: Cycle: a collection of edges in $E$ that forms a negative cycle.

\section{Initialization:}

1 Distance $\leftarrow$ []; the array for storing minimal distances from source to each vertex.;

2 PredecessorEdge $\leftarrow$ []; the array for storing predecessor edge for each vertex;

Algorithm:

RelaxedBellmanFord $(G, \epsilon)$

3 // Initialize distance and predecessor array;

......

4 // Update distances from source to each vertex, only if the distance decreased by at least $\epsilon$;

5 for $i \in[1, \operatorname{LEngth}(V)-1]$ do

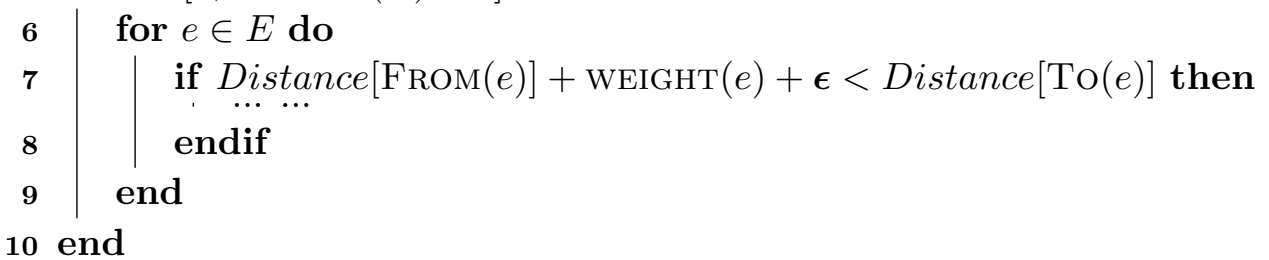

\section{0 end}

11 // Extract negative cycle, if exists;

12 for $e \in E$ do

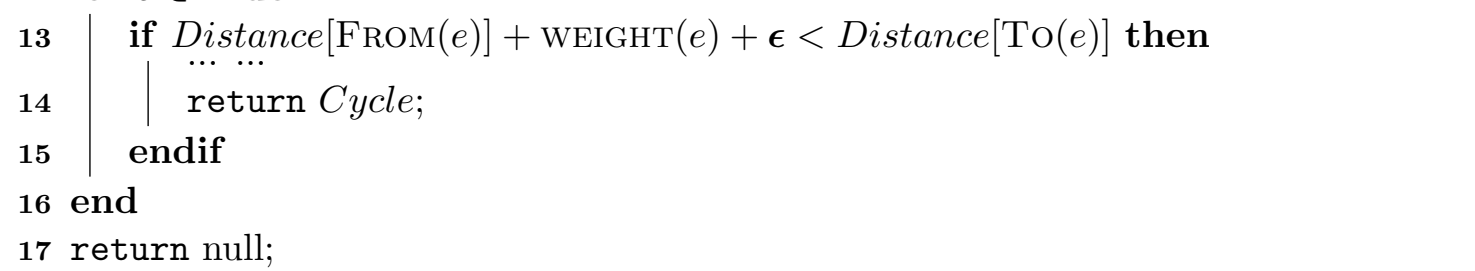

Algorithm 8: Bellman-Ford algorithm with relaxed criteria on distance updates

help find new conflicts. For example, when solving a simple over-constrained STN from the bus scheduling domain (discussed in the next section) with 417 events and 672 constraints, 381 continuous relaxation operations were executed by BCDR in order to discover the 381 conflicts and find the optimal solution. Out of the 17.01 seconds run-time, 13.14 seconds were consumed by computing continuous relaxation, and the one we are mostly interested in is the last one when we have learned all conflicts. The process is visualized in Figure 22 , in which we plot the cumulated runtime against the number of continuous conflicts discovered, and the continuous relaxation computation time against the number of conflicts it is resolving. To improve the efficiency of this procedure, the key is to discover new conflicts without incurring so many expensive operations for computing optimal continuous relaxation, such that the run time does not increase as fast when discovering new conflicts.

Therefore, we developed a greedy approach for resolving continuous conflicts during search: picking the constraints with the lowest relaxation cost in a conflict, and relaxing their lower or upper bounds to the extent that the linear expression of the conflict is nonnegative. The exact continuous relaxation procedure only needs to be called to refine 


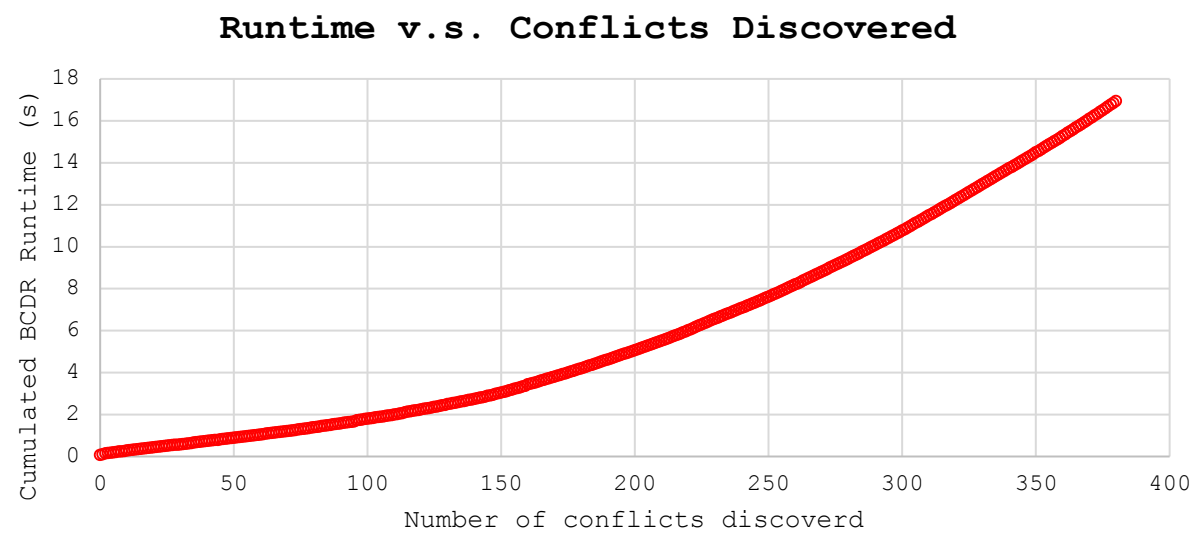

(a)

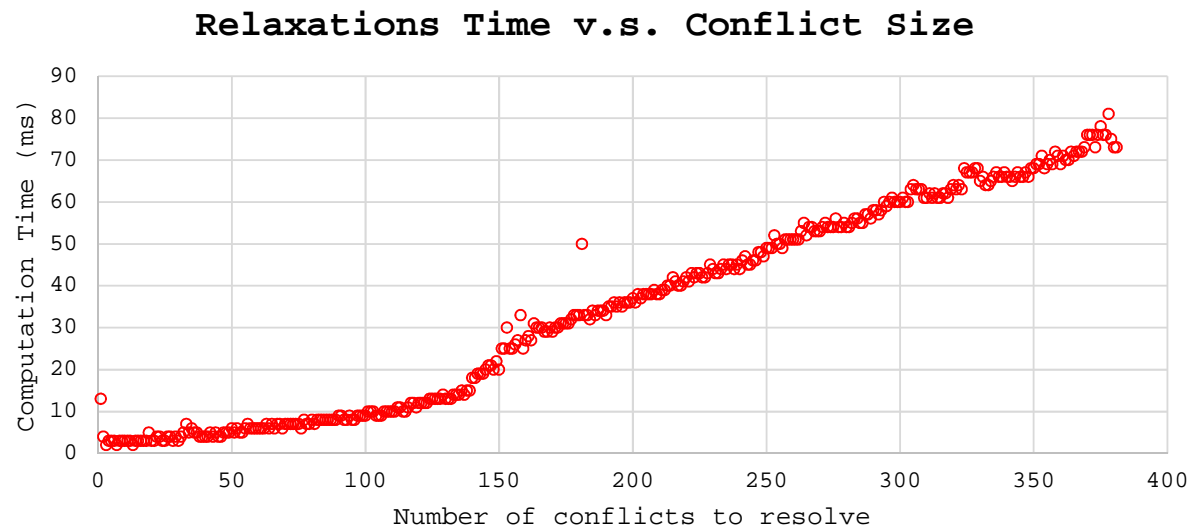

(b)

Figure 22: Profile of continuous relaxations in BCDR runtime

the relaxations when a consistent set is found: during the search we may use the greedy approach for a new candidate that can push BCDR to discover new conflicts. It takes very little time compared to the exact optimal relaxation, since it does not require calling the optimizer. It has significantly improved BCDR's runtime performance on large-scale and highly constrained problems. For the same over-constrained problem, this greedy relaxation approach reduces the runtime to 7.2 seconds, within which only 0.89 second were spent on conflict resolution. The same relaxation time and plot are shown in Figure 23. Each point in Figure 23b represents the discovery of one conflict. The closer it is to the x-axis, the less time was spent on computing continuous relaxations after discovering the conflict. As can be seen in the graph, less than ten exact relaxations were computed to refine the greedy relaxations on this problem, which greatly reduces BCDR's run time: greedy relaxations were used in most situations to discover new conflicts. The downside is that we discovered more conflicts than before (566 vs 381 ), which may not be necessary for generating the optimal relaxation.

The greedy approach requires very minimum modification to BCDR: instead of formulating and solving the optimization problem in EXPANDONCONFLICT, we compute the 


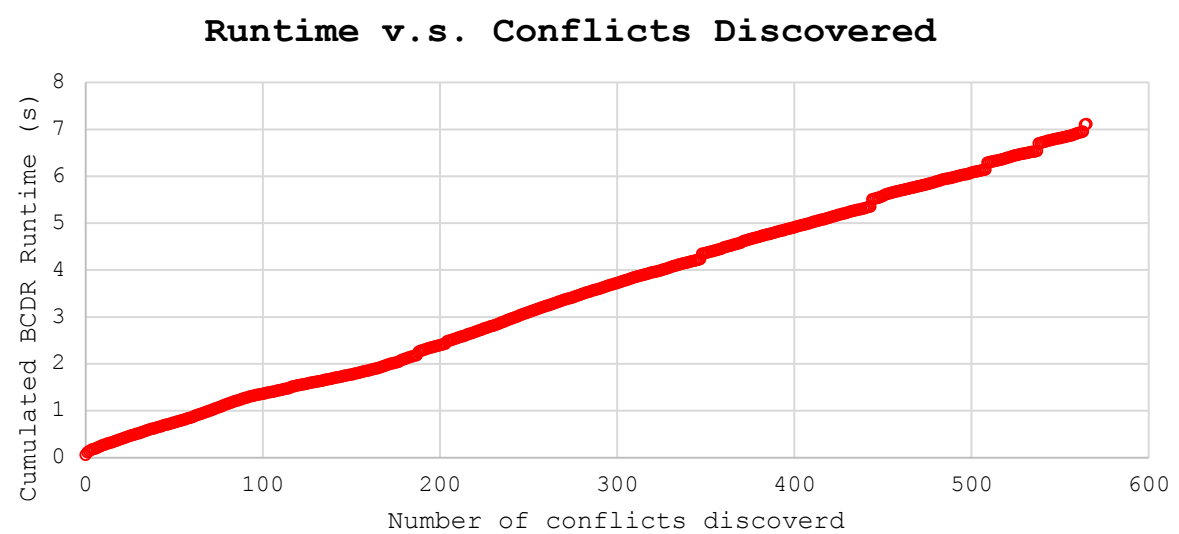

(a)

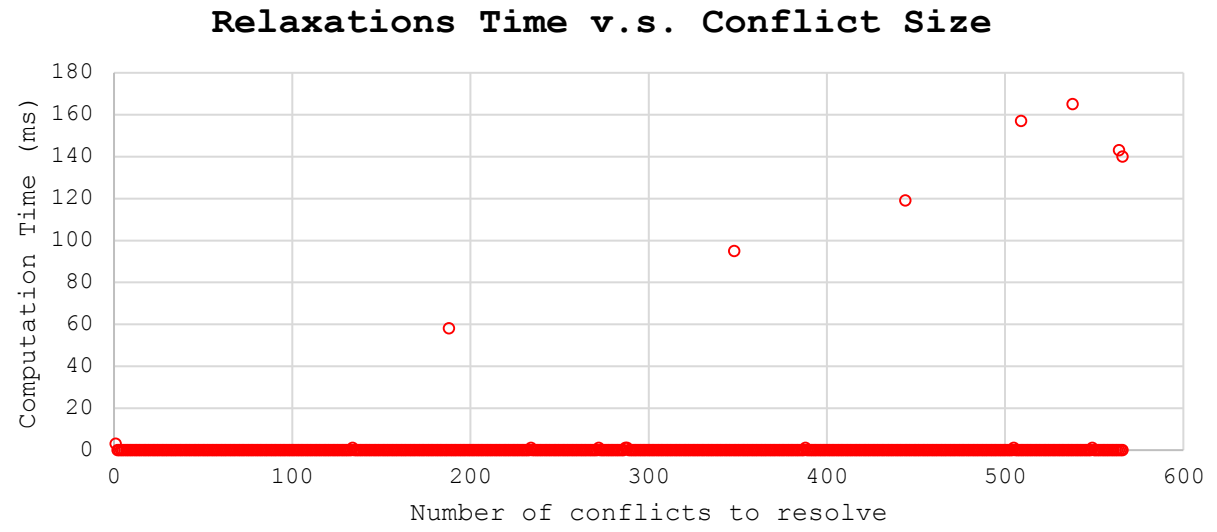

(b)

Figure 23: Profile of combined greedy/exact relaxations in BCDR runtime

greedy relaxations here. Inside ExPANDOnCONFLiCT, Line 16 to Line 22 (Algorithm 2) are replaced with a new procedure that only looks at the new conflict currConf (Algorithm 9), instead of all conflicts that were previously resolved continuously. The greedy relaxation procedure iterates through all constraints in the conflict and checks if any constraints involved are relaxable (Line 16-17). If such a constraint is identified, it will relax its bounds, either lower or upper, to the maximum extent or to the extent that the conflict is eliminated, whichever is smaller (Line 18). The initial value of the conflict's linear expression is captured by the variable Offset, and the conflict is resolved once the variable is made zero or negative. If one constraint cannot provide enough deviation, the procedure will move on to the next one, until Offset is made zero or less. If the loop completes but the offset is still positive, it means that no continuous relaxation is available for resolving the new conflict, and the continuous relaxation candidate will not be generated.

The optimal relaxation procedure is moved outside of ExPANDONCONFLICT, and to the main loop of BCDR. Once BCDR has verified the feasibility of a candidate solution, instead of retuning it, an additional procedure will be executed to check if the candidate contains any greedy relaxations (Line 13, Algorithm 10). If true, it will refine the relaxations 


\section{Initialize}

Offset $\leftarrow 0$ - EvalExp $($ currConf $)$; amount of relaxation that need to be applied for resolving the conflict continuously;

......

16 for $c \in \operatorname{curr} C o n f$ do

17 if IsRelaxable $(c)$ then

$18 \quad r=\operatorname{Min}($ Offset, RelaxationLimit $(c))$;

$19 \quad R_{\text {new }} \leftarrow R_{\text {new }} \cup\{\langle c, \mathrm{LB}(c)-r, 0\rangle\}$ or $\{\langle c, 0, \mathrm{UB}(c)+r\rangle\}$;

$20 \quad$ Offset $=$ Offset $-r$;

21 end

22 if Offset $\leq 0$ then

$23 \quad$ Cand $_{\text {new }} \leftarrow\left\langle A, R_{\text {new }}, C_{r}, C_{\text {cont }}\right\rangle$;

$\mathbf{2 4} \quad$ newCands $\leftarrow$ newCands $\cup$ Cand $_{\text {new }}$;

25

26 end

27 end

Algorithm 9: Modifications to Function ExPANDOnConflict (Algorithm 2) for handling candidates with greedy relaxation

using the optimization procedure and generate a new candidate. The new candidate will be put back to the queue for further verification, since it may not be the best or consistent candidate after the updates to its continuous relaxation. The greedy approach always underestimates the cost of resolving conflicts, ensuring that the heuristic function of BCDR remains admissible. This refinement step corrects such underestimation before returning candidates, allowing BCDR to enumerate resolutions in best-first order with delayed conflict resolution.

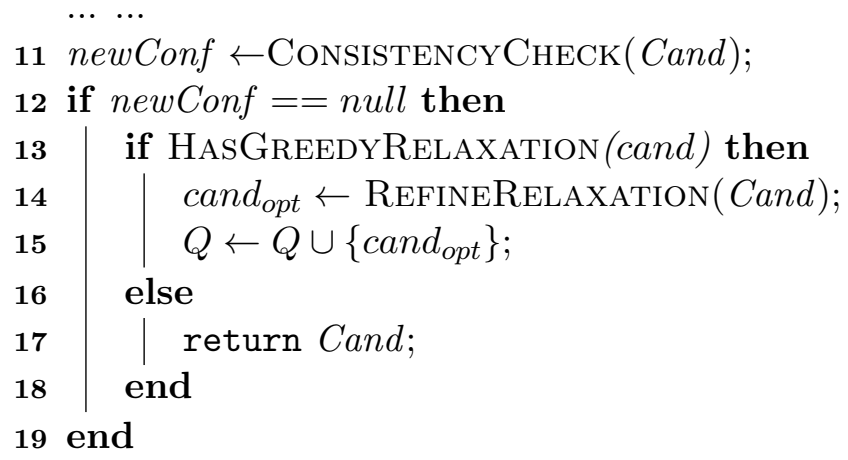

Algorithm 10: Modifications to the BCDR algorithm (Algorithm 1) for greedy continuous relaxation

This approach works very well on non-conditional problems, whose search tree has one single branch. However, it may not save time on conditional ones. To enumerate candidate solutions in best-first order, BCDR needs an accurate estimation for the cost of relaxation 
in order to prioritize candidates. In order to keep the heuristic function admissible, the cost for all greedy relaxation are set to zero. This is because the greedy relaxations have larger than optimal cost, and we have no idea how much the 'minimal cost' will be. Hence, setting the cost to be zero retains the admissible property and still allows BCDR to do a best-first enumeration. However, if we stick to the greedy relaxations, the utility estimation for candidates may be off by a large margin, causing BCDR to waste a lot of time examining candidate solutions that are far from the optimal.

Therefore, we propose a mixed greedy-optimal relaxation approach to achieve a good balance between efficiency and accuracy. The new approach uses greedy relaxation when more conflicts are likely to be discovered, and only runs the continuous relaxation procedure if we are confident that no more conflicts will be discovered, or we have been applying greedy relaxation for more than $N$ times consecutively. If a candidate is found to be consistent, or has more than $N$ greedy relaxations, the exact relaxation procedure will be used. The parameter $N$ is a non-negative integer, and if $N$ is too large then the utility estimation may be off by too much. Therefore, in our experiments we have been using $N=5$ to achieve a good balance between the time saving and the risk of creating a huge difference in heuristic and actual costs.

\section{Applications and Empirical Evaluation}

In this section, we discuss the three applications of BCDR, in the domains of deep-sea mission planning, transit vehicle scheduling, and resource-constrained project scheduling. Within each of the applications, we present the modeling of the problems, the configurations of BCDR for solving them, and the experiments for evaluating BCDR's performance compared to alternative approaches or commercial solvers. These problems are of very different structure and scale, and through these experiments, we tried to explore the strength and weakness of BCDR. Our Python implementation of BCDR used in these experiments, along with all the test cases created, can be found on GitHub: https://github.com/yu-peng/cdru.

All experiments presented in this section were conducted on a desktop computer with an Intel Core i7-2600 processor and 32GB of RAM. The SNOPT optimizer we used is version 7.2, and the Gurobi optimizer is version 6.5.1.

\subsection{Managing Deep-Sea Exploration Missions}

As presented in the beginning of the paper, the BCDR algorithm has been incorporated as part of a mission advisory system for helping ocean scientists schedule autonomous underwater vehicle tasks in deep-sea expeditions. Their missions are usually weeks long, and involve the operations of several AUVs. Each vehicle usually performs ten to fifteen dives in a mission, and a dive may last between 6 to 16 hours. During each dive, a vehicle is tasked with a set of survey locations on the sea floor: the vehicle needs to traverse between each location, take samples and images, and return before running out of power. Due to unexpected ocean currents and incomplete terrain data, the traversal times between survey sites is highly uncertain and difficult to estimate. It is almost impossible for the scientists to correctly assess the uncertainty of each dive and plan tasks accordingly to meet the risk bound and a large set of operational constraints. 
The advisor can check the feasibility of a mission plan and search for valid risk allocations that meet the risk requirement. If no such allocation can be found, it will explain the cause of failure using the conflicts detected during the search, and propose preferred relaxations to resolve the over-constrained plan. If the users are not satisfied with the results, they can ask the mission advisor to adjust the solutions given their new inputs.

We examined the performance of BCDR on problems generated from this domain, with different sizes and complexity. The test cases were created using a mission simulator for underwater expeditions. Given a set of target locations on a map, the simulator generates survey tasks around them and connects these locations with traversal activities. Each test case describes the operations of multiple AUVs over several dives, and each vehicle's dive may contain multiple survey tasks. In addition, the traversals are represented by probabilistic durations, while the survey times and battery restrictions are modeled by simple temporal constraints. The operational risk limit is specified by the chance constraints in the cc-pCCTPs. Multiple underwater robots may be deployed and working in parallel during a dive: each robot may have different speed and power storage. Depending on the distance and vehicles, probabilistic durations of different traversals and robots have different distributions.

These test problems have a very unique relay type structure: resources are being used repeatedly for different tasks, with constraints restricting the start times, end times or durations of the tasks. In addition to deep-sea exploration, this structure is also shared by problems in many other domains, such as scheduling vehicle usage in a car-sharing network.

\subsubsection{SETUP}

The randomly generated mission cc-pCCTPs have a similar structure to the example presented in Section 2. To make it more complex, we extended the problems to include multiple robots and dives: there is always another scientist waiting for the shared robot following each dive; and there are multiple robots that are operating in parallel. We use the following control parameters in the mission problem generator to characterize the complexity of a test case:

- $N_{d}$ : number of dives per robot. $1 \leq N_{d} \leq 5$.

- $N_{r}$ : number of AUVs available. $1 \leq N_{r} \leq 12$.

- $N_{a c t}$ : number of activities per dive. $1 \leq N_{\text {act }} \leq 4$.

- $N_{\text {opt }}$ : number of alternatives per activity. $2 \leq N_{\text {opt }} \leq 6$.

The total number of discrete variables in a test case is $N_{d} \times N_{r} \times N_{a c t}$, and the domain size of each variable is determined by its $N_{\text {opt }}$. Each problem is constructed as follows. We randomly sample locations from a region in Northern Pacific, within a $10 \mathrm{~km}$ radius of $(33.251,-121.555)$. The traversal times are computed using an average speed randomly selected between 10 and 20 kilometers per hour. The survey length at each location and the dive duration, $T_{a c t}$ and $T_{d i v}$, are randomly sampled in $[10,90]$ and $[60,960]$ (minutes), respectively. These durations are encoded as relaxable temporal constraints. We define linear preference functions over these relaxable constraints with gradient (cost per minute) 
sampled between 0 and 10. The reward for each variable assignment, denoting a location selection for each survey activity, ranges from 0 to 1000. For example, Figure 24 presents an overview for the structure of a test case with 2 vehicles and each carries our two activities during their dives.

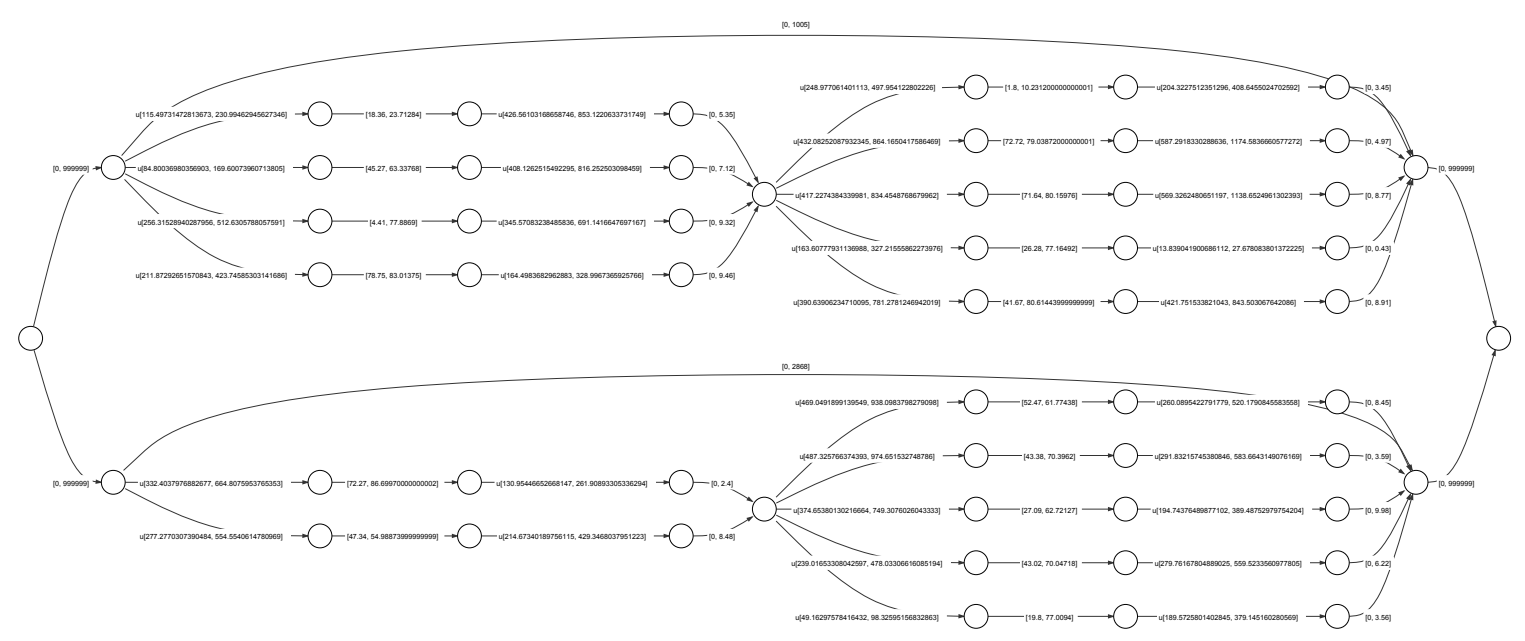

Figure 24: Overview of the structure for a test case

In total, we created 2400 test cases using randomly generated numbers of robots, risk bounds, survey locations and mission length. For each test case, we run BCDR with the following five configurations:

A. Consistency: determine and restore the feasibility of the test cases using BCDR. This algorithm is denoted as 'BCDR'. The cc-pCCTPs are treated as CCTPs by this configuration: the uncertain durations are assigned lower and upper bounds equal to their mean $[\mu, \mu]$.

B. Strong Controllability: using BCDR-U with a strong controllability model to determine and restore the feasibility of the test cases. This algorithm is denoted as 'BCDR-U(SC)'. The cc-pCCTPs are treated as CCTPUs: the uncertain durations are assigned lower and upper bounds computed from their mean and variance $[\mu-3 \sigma, \mu+3 \sigma]$.

C. Dynamic Controllability: using BCDR-U with a dynamic controllability model to determine and restore the feasibility of the test cases. This algorithm is denoted as 'BCDRU(DC)'. The cc-pCCTPs are treated as CCTPUs: the uncertain durations are assigned lower and upper bounds computed from their mean and variance $[\mu-3 \sigma, \mu+3 \sigma]$.

D. Chance-constrained Strong Controllability: using BCDR-C to find a grounded STNU of the cc-pCCTP that is strongly controllable while meeting the chance constraint, or a set of relaxations for the cc-pCCTPs that will enable such a STNU. This algorithm is denoted as 'BCDR-C(SC)'.

E. Chance-constrained Dynamic Controllability: using BCDR-C to find a grounded STNU of the cc-pCCTP that is dynamically controllable while meeting the chance constraint, 
or a set of relaxations for the cc-pCCTP that will enable such a STNU. This algorithm is denoted as 'BCDR-C(DC)'.

\subsubsection{Results}

In this experiment, we benchmarked BCDR and its variants on each problem using the five aforementioned configurations. We use SNOPT as the linear optimizer for consistency and controllability based tests, and non-linear optimizer for chance-constrained tests with probabilistic durations. In each test run, the time consumption until the first solution returned, numbers of conflicts detected, as well as the utility of the solution, were recorded. The timeout for each run was set to be 30 seconds, which is usually the maximum duration scientists are willing to wait for.

First, we present the results for Configuration A, B and C, which are BCDR, BCDR$\mathrm{U}(\mathrm{SC})$ and BCDR-U(DC). The runtime performance of the three algorithms are presented in Figure 25. Each dot in the graph represents the cumulative number of instances solved on problems that contains equal or less number of constraints, which are indicated by the $\mathrm{x}$-axis. In total, BCDR with temporal consistency checker solves 1589 problems within 30 seconds, while the number for BCDR-U(SC) and BCDR-U(DC) are only 430 and 695, respectively. As can be seen from the figures, BCDR with consistency assumption is able to solve problems with up to 1000 constraints, while the other two algorithms never succeeded with problems beyond 500 constraints. This is the result of the different handling of uncertain durations: due to the consideration of all possible outcomes from each uncertain duration, both controllability-based BCDR-U algorithms are more restrictive compared to the consistency-based BCDR, which treats uncertain durations as controllable and may only satisfy one of its many outcomes. Hence the number of conflicts detected and resolved by both BCDR-U(SC) and BCDR-U(DC) are much higher than that of BCDR (Figure 26, 27 and 28), which significantly impacts their performance on large problems.

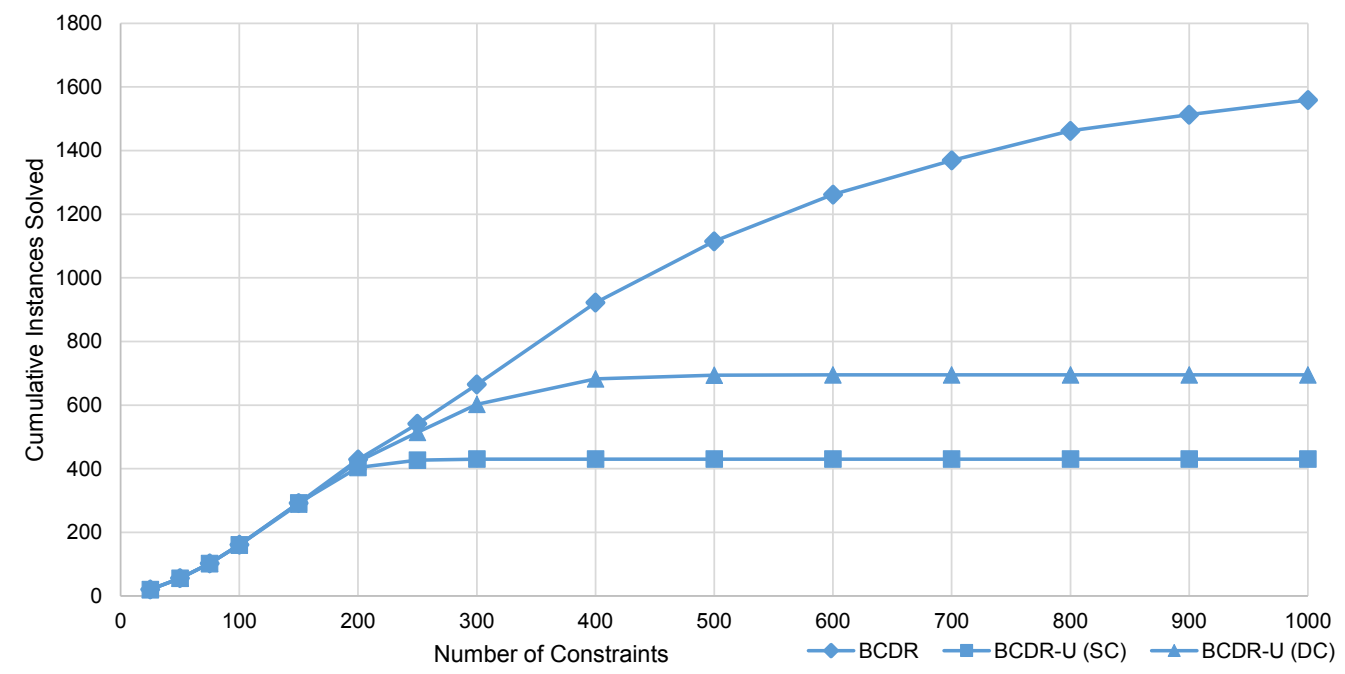

Figure 25: Cumulative number of instances solved by BCDR (in 30 seconds) 
In addition, checking strong and dynamic controllability are also more expensive operations than checking consistency, which contributed to their lower runtime performance. As presented in Section 3, we use the Bellman-Ford algorithm for consistency checking and negative loop extraction $\left(O\left(N^{2} \log N\right)\right)$. We add another layer of triangular reduction $\left(O\left(N^{2}\right)\right)$ on top of it for checking strong controllability. For dynamic controllability, we use the FASTDCCHECK algorithm $O\left(N^{4}\right)$, introduced by Morris (2006). It is interesting to observe that BCDR-U(DC) out-performs BCDR-U(SC), as there is an order-of-magnitude difference in their checking algorithms' runtime complexity. This is likely the result of the smaller amount of conflicts that were resolved by the dynamic controllability version, as shown in Figure 27 and 28. Dynamic controllability allows more flexibility than strong controllability in that it does not require a static schedule to accommodate all constraints and uncertain durations. The runtime of BCDR-U is dominated by conflict resolution for over-constrained problems, hence the less time spent on conflict resolution compensated for the additional time on controllability checking.

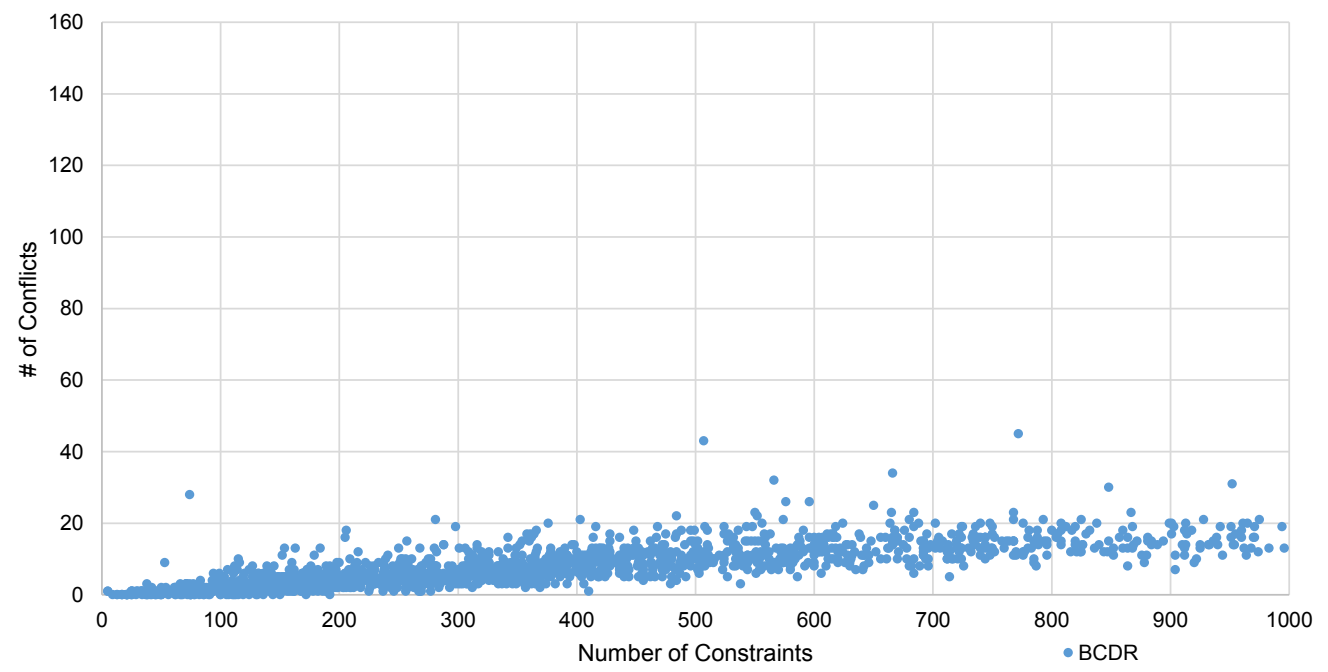

Figure 26: Conflicts detected by BCDR (Consistency)

Next, we discuss the results of BCDR-C(SC) and BCDR-C(DC) (Configuration D and E), the two chance-constrained relaxation algorithms with strong and dynamic controllability checker. They were used to restore the feasibility of cc-pCCTPs through both temporal and chance constraints relaxations. The results is shown in Figure 29.

In total, 269 of 2400 tests were solved by BCDR-C(SC) in 30 seconds, while the number for BCDR-C(DC) is 328 of 2400. Similar to the BCDR-U experiment, the dynamic controllability version performs better than the strong controllability version due to the smaller number of conflicts. BCDR-C solves fewer problems than BCDR-U and BCDR within the same time limit. This is mainly due to BCDR-C's chance-constrained conflict resolution procedure: it adds a non-linear constraint for risk allocation to the optimiation problem, which makes it significantly harder to solve than the linear optimization problem required by BCDR and BCDR-U. As can be seen in Figure 30 and 31, the number of conflicts detected by BCDR-C are not larger than those detected by BCDR-U (Figures 27 and 28). 


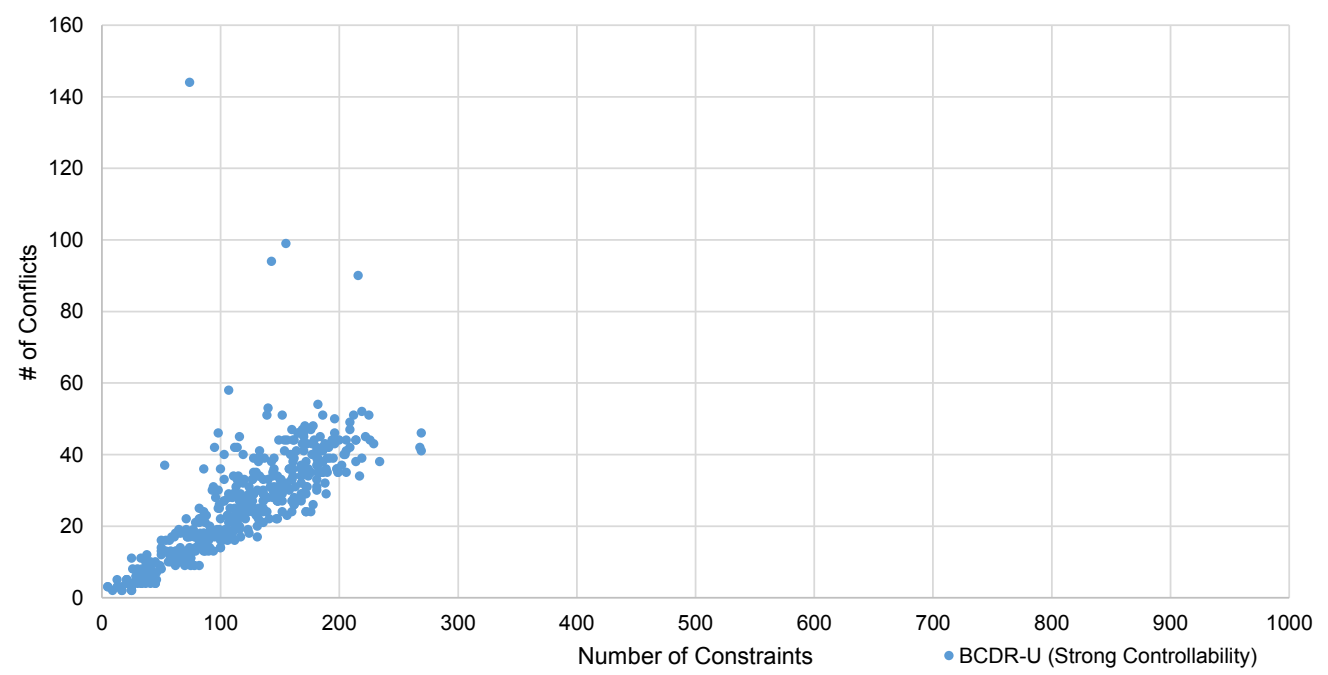

Figure 27: Conflicts detected by BCDR-U(SC)

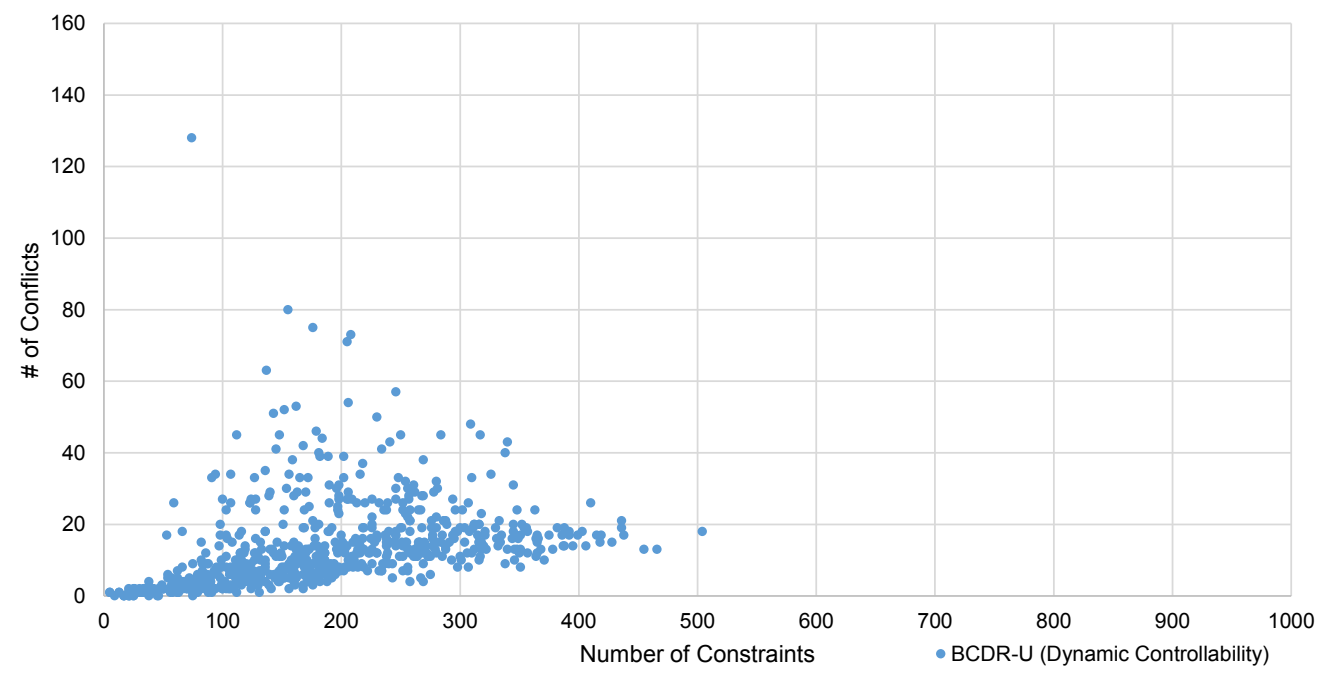

Figure 28: Conflicts detected by BCDR-U(DC)

Solving the optimization problems for conflict resolution is the most expensive operation in the BCDR algorithm, which takes up to $90 \%$ of the total computation time. Hence the extra computation was mainly due to the expensive non-linear optimization process.

BCDR performs much better on problems with set-bounded uncertainty models, since all constraints are linear during conflict resolution. On the other hand, non-linear models, such as normal distribution, is better for describing the uncertainty in some real world activities (e.g. the timing of natural phenomena). Even though it requires much more computation time, BCDR-C was able to resolve most of the problems with less than 200 constraints in 30 seconds. This is enough for modeling a 10-hour survey mission of several underwater vehicles working in parallel. In general, the algorithm configuration and modeling should be different from one application to another. The choice of uncertainty and controllability 


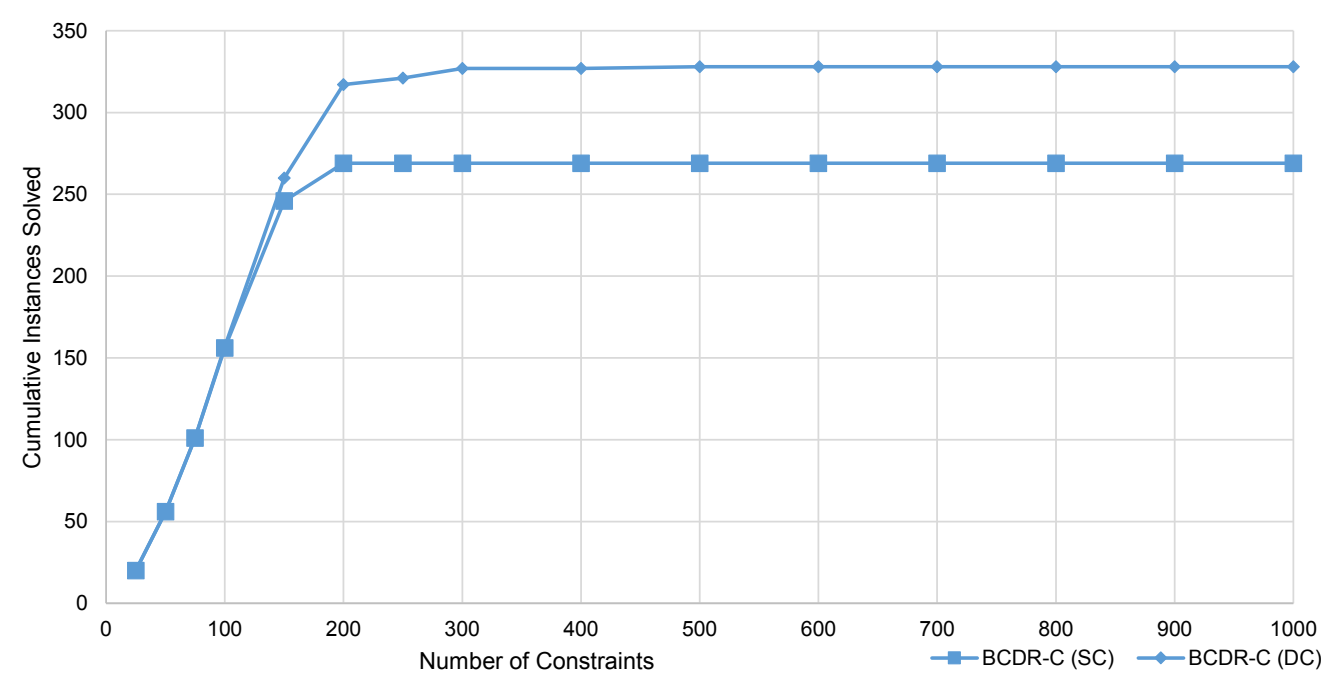

Figure 29: Cumulative number of instances solved by BCDR-C (in 30 seconds)

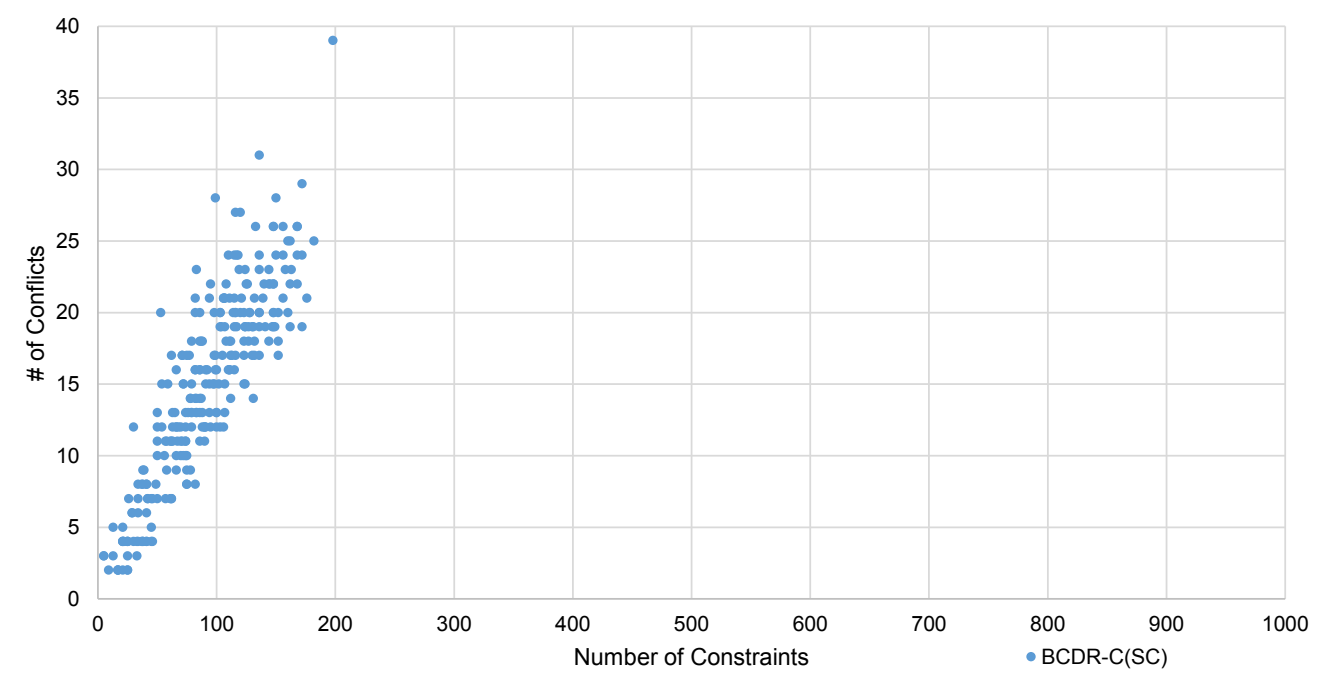

Figure 30: Conflicts detected by BCDR-C(SC)

model to use is thus application dependent. In addition, results from this experiment also suggest that tractability must be considered when selecting these models.

\subsection{Optimizing Dispatching Strategies for Maintaining Headways on Transit Routes}

In this section, we discuss a different application of BCDR in the domain of transit system management. Due to unexpected delay in travel and dwelling, transit vehicles sometimes cannot operate on schedule and maintain their designated headways. If a vehicle is delayed and operating off schedule, the gap between it and an earlier vehicle will increase, causing it to carry more passengers, spend more time at each station for dwelling and get 


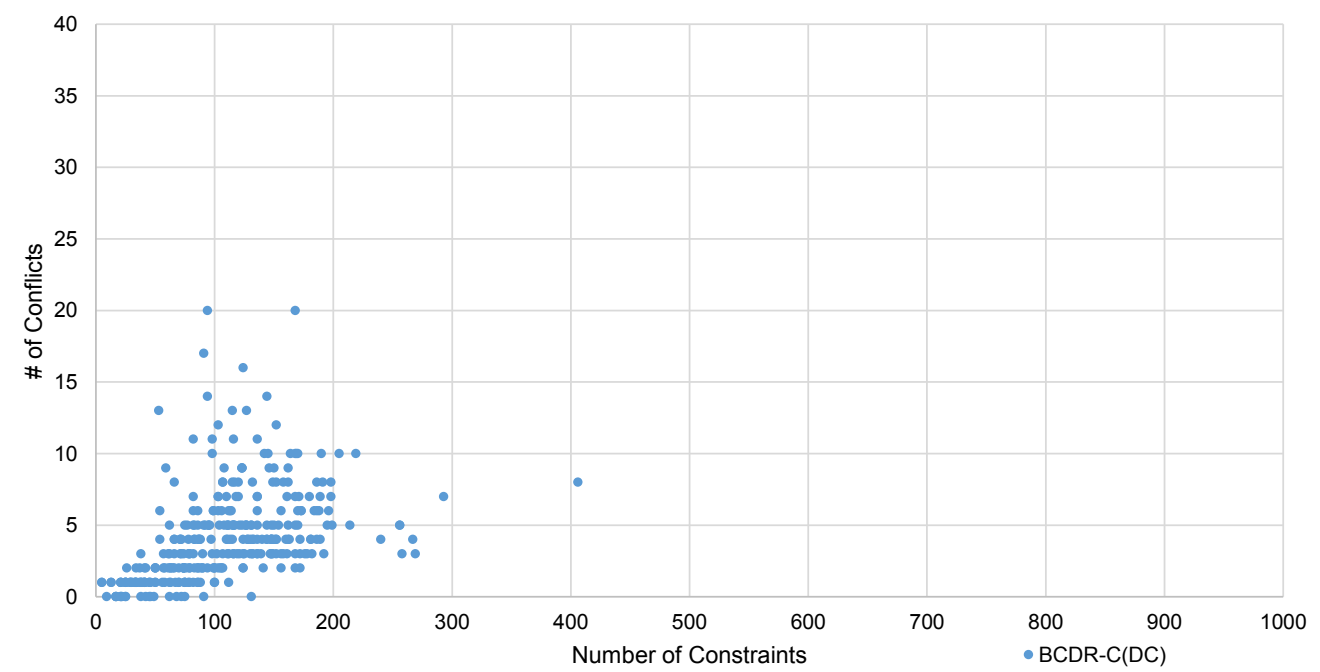

Figure 31: Conflicts detected by BCDR-C(DC)

delayed even more. In some extreme scenarios, passengers waiting at a station may see two or more vehicles along the same route arrive together, and find an overcrowded vehicle followed by near-empty ones. This problem is often called bunching or platooning in public transportation, and is a major challenge for reliable transit service.

This problem has been investigated by many researchers in operation research, and Bellei and Gkoumas (2010) present more details about it. Here we present a simple example, constructed based on an SBS Transit article on bus bunching ${ }^{1}$, to explain this problem. Assume that three vehicles are running in 8-minute intervals and demand along the route is constant (Figure 32).

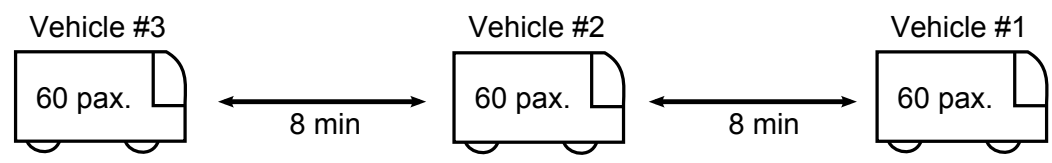

Figure 32: Regular headways between vehicles

Vehicle \#1 and \#3 did not experience any delay in its operation and was able to keep to schedule. Vehicle \#2 encountered some issues while dwelling at a previous station. Hence, the headway between Vehicle \#1 and \#2 was lengthened, while the headway between Vehicle \#2 and \#3 was shortened (Figure 33).

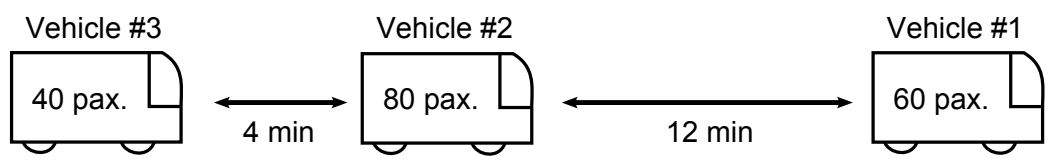

Figure 33: Uneven headways and passenger load due to delayed Vehicle \#2

1. 'Sometimes, two buses of the same service arrive at the same time with one bus being overcrowded and the other almost empty. Why?', http://www.sbstransit.com.sg/doyouknow/facts_bus.aspx. 
By taking more than its share of passengers Vehicle \#2 slowed down as a result, while Vehicle \#3 picked up fewer passengers. As this continues, Vehicle \#2 would eventually bunch up with Vehicle \#3. To a passenger waiting for Vehicle \#2, he would have waited for 6 minutes, and it would seem that Vehicle \#2 was crowded while Vehicle \#3 was relatively empty.

Current approaches for preventing vehicle bunching heavily rely on the operators' experience and intuition: they have to respond quickly enough to any irregular operations before they propagate to the entire route. In addition, the operators have a very limited set of actions to take, such as asking the delayed buses to skip stations or urge the passengers to wait for the next bus. Both may cause inconvenience for passengers either on board or waiting at stations.

Here, we present a dynamic scheduling approach for managing a transit route to address this problem. Given the schedule of an existing transit route and historical performance data, we use BCDR to compute a robust dispatching strategy for the vehicles on this route, such that the headways between buses/trains can be better maintained. It builds in additional buffer time for vehicles to wait at each station based on the uncertainty, which allows a dynamically controllable dispatching strategy to be generated. The dispatching strategy, represented as an execution policy for events in the scheduling problem, provides real-time guidance for pausing vehicles at each station in order to maintain headways along the route. For the passengers, it means that the frequency of services is more regular, such that they are less likely to wait for an extended period of time for the service, or board an overly crowded vehicle.

Our approach is similar to the frequency-based method proposed by Bartholdi and Eisenstein (2012), which has each bus observe the preceding and following ones, and strategically delay themselves at stations to maintain regular headways. As presented in the same paper, this method has been shown to outperform prior work in controlling the university bus system at Georgia Institute of Technology. The major difference between it and our scheduling based approach is that we used a centralized algorithm and pre-compute the strategy based on historical data. It allows us to coordinate all vehicles along the route simultaneously, and restore from service interruptions quickly without waiting for the bus delays to propagate from one to another. However, the trade-off is that the dynamic policy requires more computation time to generate, and the policy itself may become invalid if any travel or dwell time along the route falls outside the set-bounds for uncertain durations.

The objective of this experiment is to explore different problems that BCDR can solve and benchmark its scalability. The work presented in this section is not part of any research project, and results have not been compared to other approaches in the field. We have not verified this approach on any real transit routes, though we would very much like to share it and evaluate this approach in a real-world system.

\subsubsection{SETUP}

We selected the Red Line subway ${ }^{2}$ in Boston for this experiment. It is the busiest mass transit route in the city, carrying more than 250,000 passengers per day. The agency operating Red Line, the Massachusetts Bay Transit Authority, has been publishing performance

2. http://www.mbta.com/schedules_and_maps/subway/ 
data for every train operation since June 30, 2015. Using their API, we were able to retrieve the travel times of each train between stops, and the amount of time the train dwelled at every stop. Combined with the published schedule, we constructed a CCTPU for modeling the operation of the Red Line. To simplify the problem, our model covers only the inbound direction trains from Alewife station to JFK/UMass station (before the line branches into two directions).

The travel time of each train between stations and dwell times are represented by uncertain durations, while the scheduled headways are encoded as simple temporal constraints. Figure 34 is cropped from the visualization of the problem, which shows a small portion of the CCTPU that presents all basic elements: dwell, wait for departure, and traversal to the next station. Given a train ride, the three elements are repeated for each station pair. The collection of constraints for one train ride are then repeated for all 167 trains during a peak day along the inbound direction. Between neighboring trains, the headway constraints are added between their their arrival events at a station. The temporal bounds of these constraints are defined using the scheduled headway: [Headway -1, Headway +1$]$. We slightly weakened the headway requirements from the schedule by \pm 1 minute to allow some flexibility for handling uncertainty, such that more efficient solutions can be generated.

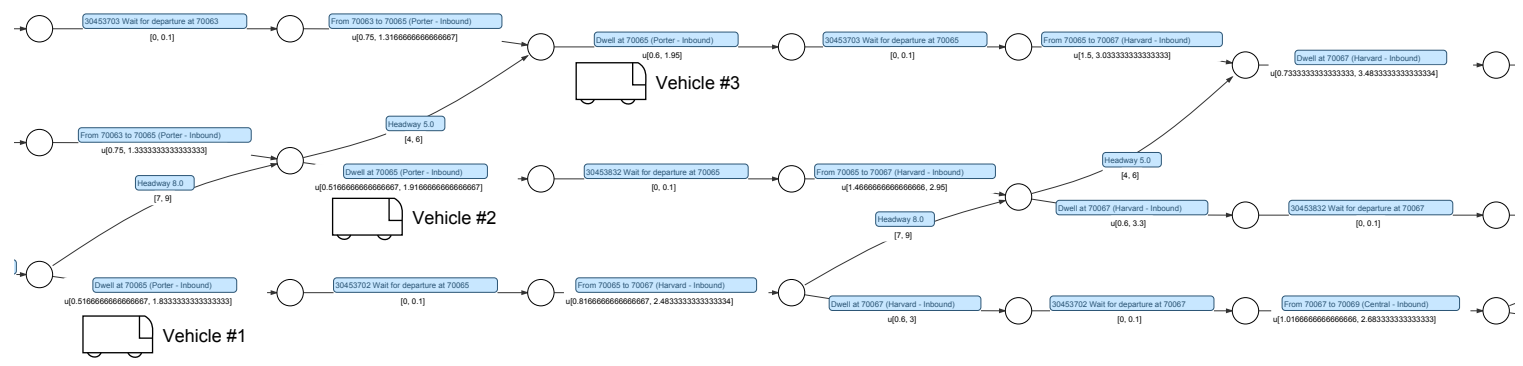

Figure 34: Basic elements in the CCTPU for Red Line trains

The bounds for the uncertain durations, travel times, and dwell times are estimated using the historical performance data: given a train ride between station $\mathrm{A}$ and $\mathrm{B}$, we retrieve train operation data between the same stations in history and compute a lower and upper bound to cover them. For example, for a train ride that leaves station Alewife at 5pm, we will retrieve all records for previous trains that left between 4:30pm to 5:30pm during weekdays, and collect their travel times between stations and dwell times. For its uncertain duration, the lower bound is defined using the smallest travel time in the collection, while the upper bound is chosen such that $98 \%$ of all data points are covered between the lower and upper bounds.

Given the uncertain durations, there is no execution policy that meets all constraints in the CCTPU. The objective of this experiment is to restore the dynamic controllability of the over-constrained CCTPU, by building in the minimal amount of wait times at each station. We can then compute a dispatching strategy that is robust against all uncertain travel and dwell times can be found from the relaxed CCTPU. Therefore, the only relaxable elements in the CCTPU are the upper bounds of the wait times at each station. The values of the upper bounds are initialized to be 0.01 minute, and associated with a linear cost function with gradient 1 to penalize any excessive delays. 


\subsubsection{Results}

In this experiment, we solved the over-constrained problems using two approaches: conflictdirected relaxation with BCDR-U(DC), and a MIP encoding with Gurobi. For BCDR$\mathrm{U}(\mathrm{DC})$, we use Gurobi as its sub-solver to compute optimal relaxations for learned conflicts. For the MIP encoding approach, we use Gurobi directly to solve the complete problem. The MIP encoding we used was first introduced by Wah and Xin (2007) for checking dynamic controllability, and later a modification was presented by Cui et al. (2015) to evaluate the robustness of schedules for resource-constrained project scheduling problems. The objective function for both approaches are set to find the minimum cost relaxations, which correspond to the minimum delays that have to be built into each station, and makes the CCTPU for the transit route a dynamically controllable network.

Different from the AUV mission planning problems, the transit operation problem's temporal network is highly connected due to the headway constraints. They create many more cycles in the network, which result in more conflicts between constraints. The larger number of conflicts makes the problems significantly more difficult to solve. Therefore, given the limited computing resources and experiment time, we do not expect both approaches to solve the complete problem with 169 trains and around 10,000 constraints. To benchmark and compare the performance of two approaches, we generated a set of smaller test problems by capturing only a subset of the trains and stations. Each of the 48 test problems contains $\mathrm{N}(2 \leq \mathrm{N} \leq 7)$ trains and $\mathrm{M}(2 \leq \mathrm{M} \leq 9)$ stops. In this experiment, we set the timeout of each test run to be 10 minutes.

\begin{tabular}{|c|c|c|c|c|c|c|c|c|}
\hline 7 & 182.62 & $\mathrm{x}$ & $\mathrm{x}$ & $\mathrm{x}$ & $\mathrm{x}$ & $\mathrm{x}$ & $\mathrm{x}$ & $\mathrm{x}$ \\
\hline 6 & 87.051 & $\mathrm{x}$ & $\mathrm{x}$ & $\mathrm{x}$ & $\mathrm{x}$ & $\mathrm{x}$ & $\mathrm{x}$ & $\mathrm{x}$ \\
\hline 5 & 27.012 & 314.55 & $\mathrm{x}$ & $\mathrm{x}$ & $\mathrm{x}$ & $\mathrm{x}$ & $\mathrm{x}$ & $\mathrm{x}$ \\
\hline 4 & 7.1596 & 120.48 & 370.16 & $\mathrm{x}$ & $\mathrm{x}$ & $\mathrm{x}$ & $\mathrm{x}$ & $\mathrm{x}$ \\
\hline 3 & 1.9395 & 18.362 & 57.251 & 162.20 & 424.35 & $\mathrm{x}$ & $\mathrm{x}$ & $\mathrm{x}$ \\
\hline 2 & 0.62508 & 3.2241 & 9.7885 & 19.904 & 51.379 & 80.885 & 200.97 & 346.31 \\
\hline Trains & 2 & 3 & 4 & 5 & 6 & 7 & 8 & 9 \\
\hline
\end{tabular}

Table 6: Runtime of BCDR-U with Gurobi as sub-solver (in seconds)

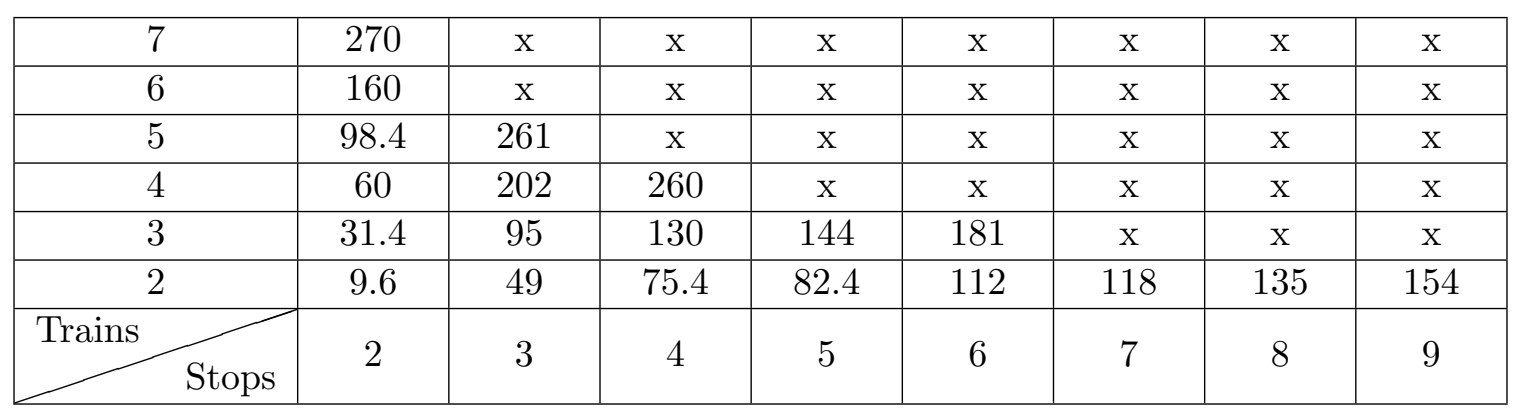

Table 7: Number of conflicts resolved by BCDR-U before finding the optimal relaxations 


\begin{tabular}{|c|c|c|c|c|c|c|c|c|}
\hline 7 & $\mathrm{x}$ & $\mathrm{x}$ & $\mathrm{x}$ & $\mathrm{x}$ & $\mathrm{x}$ & $\mathrm{x}$ & $\mathrm{x}$ & $\mathrm{x}$ \\
\hline 6 & $\mathrm{x}$ & $\mathrm{x}$ & $\mathrm{x}$ & $\mathrm{x}$ & $\mathrm{x}$ & $\mathrm{x}$ & $\mathrm{x}$ & $\mathrm{x}$ \\
\hline 5 & $\mathrm{x}$ & $\mathrm{x}$ & $\mathrm{x}$ & $\mathrm{x}$ & $\mathrm{x}$ & $\mathrm{x}$ & $\mathrm{x}$ & $\mathrm{x}$ \\
\hline 4 & $\mathrm{x}$ & $\mathrm{x}$ & $\mathrm{x}$ & $\mathrm{x}$ & $\mathrm{x}$ & $\mathrm{x}$ & $\mathrm{x}$ & $\mathrm{x}$ \\
\hline 3 & 30.399 & $\mathrm{x}$ & $\mathrm{x}$ & $\mathrm{x}$ & $\mathrm{x}$ & $\mathrm{x}$ & $\mathrm{x}$ & $\mathrm{x}$ \\
\hline 2 & 2.7040 & 12.569 & 76.568 & 340.29 & $\mathrm{x}$ & $\mathrm{x}$ & $\mathrm{x}$ & $\mathrm{x}$ \\
\hline Trains & 2 & 3 & 4 & 5 & 6 & 7 & & 8 \\
\hline
\end{tabular}

Table 8: Runtimes of Gurobi with MIP encoding (in seconds)

The results are shown in Table 6 for BCDR-U(DC), and Table 8 for Gurobi with MIP encoding. Rows indicate the number of trains captured by the test problems, while columns indicate the number of stops. The run-times of each approach for solving the test problem are the averaged results from five test runs. Within the time limit, BCDR-U(DC) solved 20 out of 48 problems with up to 7 trains/2 stops and 2 trains/9 stops, while Gurobi only solved 5 problems with less than 3 trains and 5 stops. For the problems that both methods solved in the time limit (2 Trains with 2, 3, 4, and 5 stops, and 3 Trains with 2 stops), BCDR-U(DC) is roughly one order of magnitude faster than Gurobi with MIP encoding. Similar to the results from the first experiment, the runtime of BCDR-U(DC) on an overconstrained problem is largely determined by the number of conflicts in it. As can be seen in Table 7, problems with more trains and stops generally contain more conflicts, which require longer runtime for $\mathrm{BCDR}-\mathrm{U}(\mathrm{DC})$ to solve.

The results demonstrate the advantage of BCDR-U's conflict-directed approach: it allows the solver to only deal with the conflicts instead of the complete problem, which significantly reduces the number of constraints and variables it has to consider. Although BCDR-U's conflict learning step requires a significant amount of computation, overall the procedure is still worth the effort, especially for large problems like the transit route optimization. Even for Gurobi, which is commonly regarded as a state-of-the-art MIP and LP optimizer, BCDR-U is still able to significantly improve its performance on the relaxation problems when compared to the direct MIP encoding approach.

\subsection{Robustness Analysis of Resource-Constrained Project Schedules}

Finally, we present the application of BCDR to evaluating the robustness of resourceconstrained project schedules. First presented by Cui et al. (2015), the objective of this experiment is very different from the previous two experiments: instead of over-constrained temporal problems, BCDR is given a feasible problem and a partial-order schedule for it, and is asked to find the maximum uncertainty that can be built into the durations of some activities, while maintaining the controllability of the problem. It is like finding a configuration of temporal problems that pushes them to the boundary of feasibility.

A partial-order schedule (POS) consists of a set of time constraints between activities such that any realization that meets these constraints is also resource feasible. In the deterministic case, where the duration of each activity $i$ is a constant $d_{i}$, the POS can be represented as an STN with time points $t_{s_{i}}$ and $t_{e_{i}}$ for the start and end, respectively, of each 
activity. Assuming the duration of each activity can vary within some bounds, $\left[l_{s_{i}, e_{i}}, u_{s_{i}, e_{i}}\right]$, the schedule can be modeled as an STNU where the link $e_{s_{i} e_{i}}$ from each activity's start to its end is contingent, while remaining time constraints are requirement links. Thus, given a POS, our measure of robustness is defined as the maximum deviation (i.e., width of the contingent bound) on any activity at which the STNU is dynamically controllable.

\subsubsection{SETUP}

To compute the maximum deviation allowed in a POS, we will need to slightly modify the BCDR-U algorithm and problem formulation. First, we initialize the upper bound of all activity durations to $+\infty$, which is the maximum possible uncertainty we can build in. It makes the schedule over-constrained, and allows BCDR-U to start from here to iteratively discover conflicts and restore the controllability of the schedule. Second, we modify the objective function used by BCDR-U (Equation 9) to be defined over the range of uncertain durations (Equation 14). Instead of minimizing the costs of weakening relaxable constraints, this modification allows BCDR-U to maximize the minimum deviation among all activity durations.

$$
\max \min _{u b_{i}^{\prime}}\left(u b_{i}^{\prime}-l b_{i}\right)
$$

Figure 35 presents a simple example that demonstrates the modification and the expected output from BCDR-U. Given a feasible schedule over two activities (Figure 35a), we first increase the upper bounds of the activities to $+\infty$ (Figure 35b). The change effectively applies the maximum possible deviation to the schedule, but also makes it over-constrained in most scenarios. Then we apply BCDR-U(DC) on the problem, asking it to extract conflicts introduced by the modification, resolve them by lowering the upper bound according to the new objective function, and restore the dynamic controllability of the problem (Figure $35 \mathrm{c})$.

As test cases, we use 325 partial-order schedules for RCPSP/max problems (Kolisch \& Padman, 2001) with 10-18 jobs ${ }^{3}$. The schedules are generated by a scheduler that optimises a measure of POS flexibility (Banerjee \& Haslum, 2011). The STNU representation of a schedule has a time point for the start and end of each activity, as described above. Hence, the number of nodes and contingent links is determined by the number of jobs, but the number of (given) requirement links varies from 50 to 300 .

\subsubsection{Results}

In this experiment, we solved the over-constrained problems using two approaches: conflictdirected relaxation with BCDR-U(DC), and MIP encoding with Gurobi. Similar to the second experiment on transit route schedules, we use Gurobi as the sub-solver for BCDRU(DC). For the MIP encoding approach, we used the encoding introduced by Cui et al. (2015) to compute the maximum flexibility that can be built into the uncertain durations. The problems in this experiment are of much smaller scale compared to the previous two experiments, and both approaches were able to solve all problems within the time limit (30

3. Set J10 from PSPLIB (http://www.om-db.wi.tum.de/psplib/) 


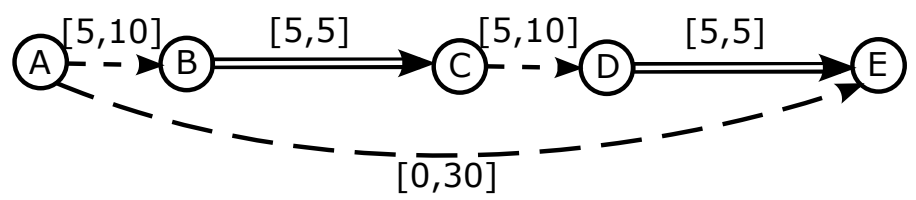

(a)

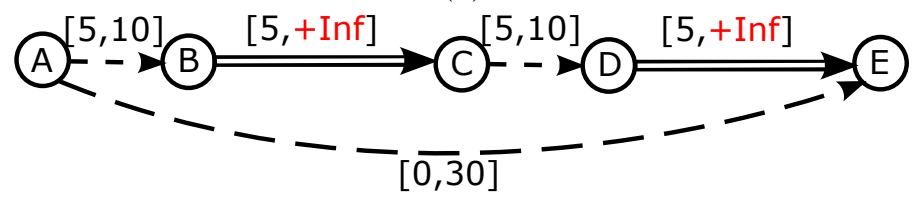

(b)

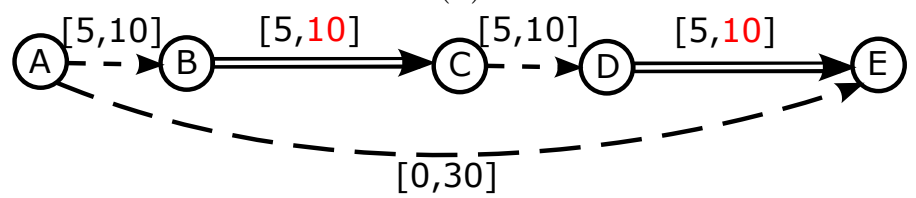

(c)

Figure 35: Examples for maximizing flexibility

seconds). For each test run, we recorded the computation times of both approaches for comparison.

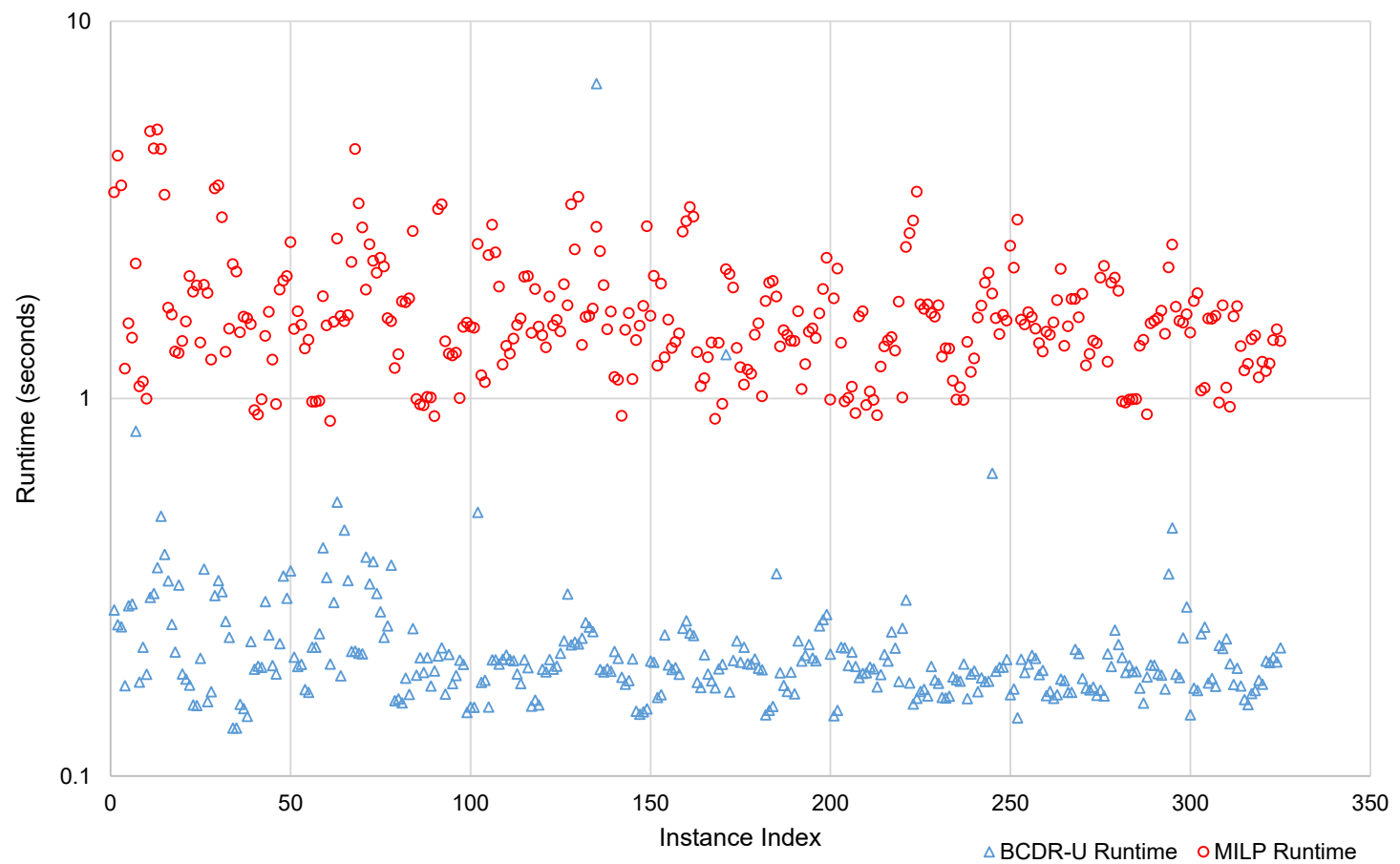

Figure 36: Runtimes of BCDR-U(DC) and Gurobi/MIP on RCPSP schedules (in seconds) 
The results are shown in Figure 36: each data point in the graph represents the averaged runtime for one problem over five test runs. Similar to the results from the previous experiments, BCDR-U(DC) is very effective for solving these problems when compared with the MIP/Gurobi approach, which is about one order of magnitude slower on most test problems.

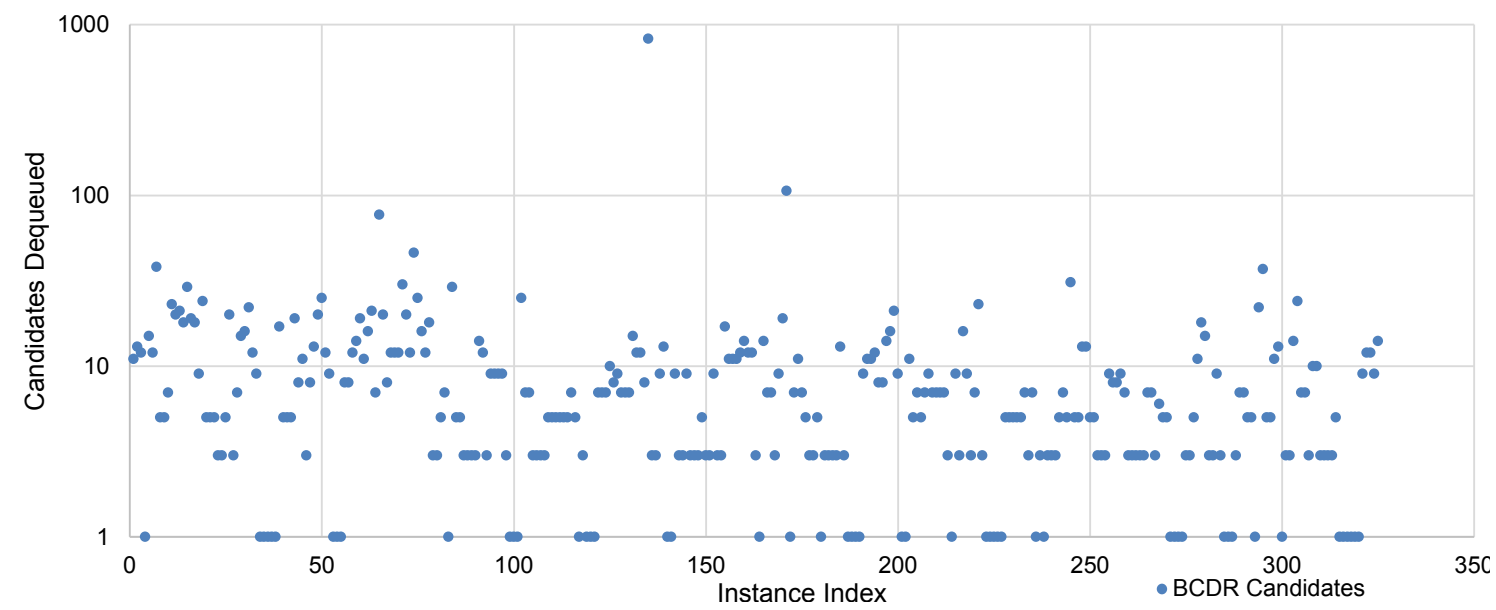

Figure 37: Numbers of candidates evaluated by BCDR-U(DC) on RCPSP schedule problems

Among the 325 test runs, we observed one interesting out-lier in the result: BCDR$\mathrm{U}(\mathrm{DC})$ spends more time solving Instance 135 than Gurobi. This is the only case in which BCDR-U(DC) spends more time, and we were interested in investigating the cause behind this issue. We started with the number of candidate relaxations evaluated in solving the problem (Figure 37). Not surprisingly, BCDR-U(DC) tested many more candidates before reaching the optimal relaxations for Instance 135 (825 candidates dequeued), which explains the longer runtime on this problem. Next, we retrieved the utility of candidates generated by BCDR-U(DC) while solving Instance 135. Figure 38 presents the utility of BCDR-U(DC)'s candidates in a test run: from the first feasible candidate (Index 1 with utility 7.6667) to the final solution (Index 825 with utility 5.1429). There are a few 'plateau' regions in the graph, which indicate that BCDR-U(DC) spent a lot of time evaluating candidates of similar utility without making progress towards the solution. This indicates one of the weakness in BCDRU(DC)'s best-first enumeration approach: without a good heuristic function for computing the bounds on continuous relaxation costs, BCDR-U(DC) may waste a large amount of time exploring parts of the search space it believes to be promising, but which in fact contain no good solutions. This issue is more often observed in problems with many conflicts, since each conflict may introduce multiple continuous relaxations and significantly enlarge the search space. Therefore, a heuristic function for estimating the bound on relaxation cost should be incorporated whenever available, and developing a general application bounding function for continuous relaxation is part of our future research. 


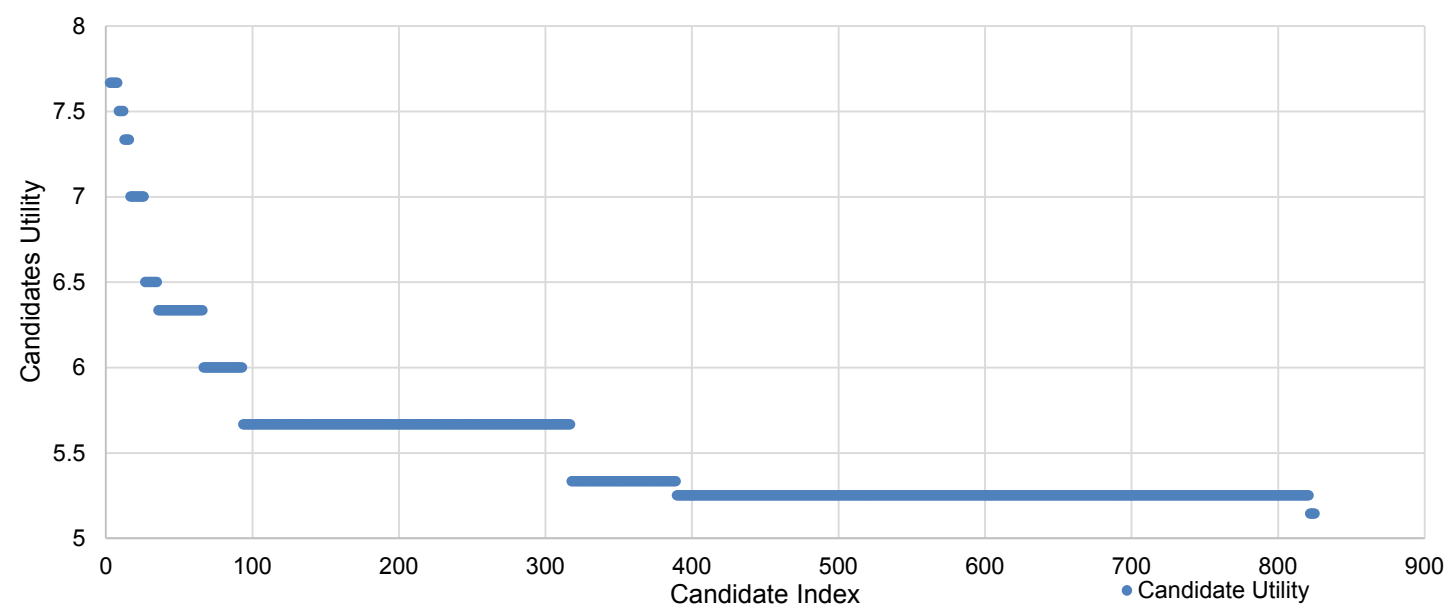

Figure 38: Utilities of candidates evaluated by BCDR-U(DC) while solving Instance 135

\section{Conclusion}

In this paper, we presented the Best-first Conflict-Directed Relaxation algorithm for resolving over-constrained temporal problems. BCDR takes a conflict-directed approach and leverages prior work on hardware diagnosis, temporal controllability checking, and chanceconstrained scheduling. Compared to previous approaches, which resolve over-constrained problems by suspending constraints, BCDR minimizes the perturbation by continuously relaxing temporal constraints to the minimal extent. With the implementation of an incremental conflict learning and resolution strategy, the algorithm is efficient at enumerating relaxations in best-first order, and can incorporate user inputs during the process.

In addition, BCDR can be easily extended to handle problems with temporal uncertainty, either expressed as set-bounded uncertain durations, or probabilistic durations with chance constraints. The two variants, BCDR-U and BCDR-C, allow the users to diagnose the source of uncertainty in an over-constrained temporal problem, and enumerate preferred resolutions with bounded risk. They help users resolve conflicts in temporal problems by trading off safety margins with performance, which provides flexibility for risk management in real world scenarios.

BCDR and its extensions have been incorporated as part of several decision support applications for deep-sea exploration, transit route management and job-shop schedule robustness analysis. Results from our experiments in these domains have demonstrated the algorithm's effectiveness in resolving large and complex problems.

\section{Acknowledgments}

We would like to thank Eric Timmons, Christian Muise, Andrew Wang, Yuening Zhang and Szymon Sidor for discussing and reviewing the work. We would like to acknowledge financial support from the Boeing Company under grant MIT-BA-GTA-1; the Defense Advanced Research Projects Agency under contract number HR0011-15-C-0098; the Defense Advanced Research Projects Agency meta program under contract number 6923548; and the Australian Research Council Discovery Project Grant DP140104219. We would like 
to thank the JAIR reviewers and editor for their insightful reviews, which helped us make significant improvements to this paper.

\section{References}

Bailey, J., \& Stuckey, P. (2005). Discovery of minimal unsatisfiable subsets of constraints using hitting set dualization. In Hermenegildo, M., \& Cabeza, D. (Eds.), Practical Aspects of Declarative Languages, Vol. 3350 of Lecture Notes in Computer Science, pp. 174-186. Springer Berlin / Heidelberg.

Banerjee, D., \& Haslum, P. (2011). Partial-order support-link scheduling. In Proceedings of the Twenty-First International Conference on Automated Planning and Scheduling (ICAPS-2011), pp. 307-310.

Barták, R., \& Cepek, O. (2007). Temporal networks with alternatives: Complexity and model. In Proceedings of the Twentieth International Florida AI Research Society Conference (FLAIRS-2007), pp. 641-646.

Bartholdi, J. J., \& Eisenstein, D. D. (2012). A self-coördinating bus route to resist bus bunching. Transportation Research Part B: Methodological, 46(4), 481-491.

Beaumont, M., Sattar, A., Maher, M., \& Thornton, J. (2001). Solving overconstrained temporal reasoning problems. In Proceedings of the 14 th Australian Joint Conference on Artificial Intelligence (AI-2001), pp. 37-49.

Bellei, G., \& Gkoumas, K. (2010). Transit vehicles headway distribution and service irregularity. Public transport, 2(4), 269-289.

Bellman, R. (1956). On a routing problem. Tech. rep., DTIC Document.

Cui, J., Yu, P., Fang, C., Haslum, P., \& Williams, B. C. (2015). Optimising bounds in simple temporal networks with uncertainty under dynamic controllability constraints. In Proceedings of the Twenty-fifth International Conference on Automated Planning and Scheduling (ICAPS-2015), pp. 52-60.

de Kleer, J., \& Williams, B. C. (1987). Diagnosing multiple faults. Artificial Intelligence, 32, 97-130.

Dechter, R., Meiri, I., \& Pearl, J. (1991). Temporal constraint networks. Artificial Intelligence, 49, 61-95.

Durrett, R. (2010). Probability: Theory and Examples. Cambridge university press.

Effinger, R., \& Williams, B. (2005). Conflict-directed search through disjunctive temporal plan networks. CSAIL Research Abstracts - 2005, 1.

Effinger, R. T. (2006). Optimal temporal planning at reactive time scales via dynamic backtracking branch and bound. Master's thesis, Massachusetts Institute of Technology.

Falda, M., Rossi, F., \& Venable, K. B. (2010). Dynamic consistency of fuzzy conditional temporal problems. Journal of Intelligent Manufacturing, 21, 75-88.

Fang, C., Yu, P., \& Williams, B. (2014). Chance-constrained probabilistic simple temporal problems. In Proceedings of the Twenty-Eighth AAAI Conference on Artificial Intelligence (AAAI-2014), pp. 2264-2270. 
Ford, L. R. (1956). Network Flow Theory. Rand Corporation.

Hunsberger, L., Posenato, R., \& Combi, C. (2012). The dynamic controllability of conditional stns with uncertainty. In Proceedings of the Planning and Plan Execution for Real-World Systems: Principles and Practices (PlanEx) Workshop, pp. 121-128.

Kall, P. (1976). Chance constrained programming. In Stochastic Linear Programming, pp. 79-92. Springer.

Khatib, L., Morris, R., Morris, R., \& Rossi, F. (2001). Temporal constraint reasoning with preferences. In Proceedings of the 17th International Joint Conference on Artificial Intelligence (IJCAI-2001), pp. 322-327.

Kolisch, R., \& Padman, R. (2001). An integrated survey of project scheduling. OMEGA International Journal of Management Science, 29(3), 249-272.

Lanz, A., Posenato, R., Combi, C., \& Reichert, M. (2015). Simple temporal networks with partially shrinkable uncertainty. In Proceedings of the International Conference on Agents and Artificial Intelligence-Volume 2, pp. 370-381.

Li, H., \& Williams, B. (2005). Generalized conflict learning for hybrid discrete/linear optimization. In Proceedings of the 11th International Conference on Principles and Practice of Constraint Programming, pp. 415-429.

Liffiton, M., Moffitt, M., Pollack, M., \& Sakallah, K. (2005). Identifying conflicts in overconstrained temporal problems. In Proceedings of the 19th International Joint Conference on Artificial Intelligence (IJCAI-2005), pp. 205-211.

Moffitt, M. D., \& Pollack, M. E. (2005). Partial constraint satisfaction of disjunctive temporal problems. In Proceedings of the 18th International Florida Artificial Intelligence Research Society Conference (FLAIRS-2005), pp. 715-720.

Morris, P. (2006). A structural characterization of temporal dynamic controllability. In Proceedings of the 12th International Conference on Principles and Practice of Constraint Programming (CP-2006), pp. 375-389.

Morris, P. (2014). Dynamic controllability and dispatchability relationships. In Integration of $A I$ and OR Techniques in Constraint Programming, pp. 464-479. Springer.

Morris, P., \& Muscettola, N. (2005). Temporal dynamic controllability revisited. In Proceedings of the 20th National Conference on Artificial Intelligence (AAAI-2005), pp. $1193-1198$.

Peintner, B., Moffitt, M. D., \& Pollack, M. E. (2005). Solving over-constrained disjunctive temporal problems with preferences. In Proceedings of the 15th International Conference on Automated Planning and Scheduling (ICAPS-2005), pp. 202-211.

Peintner, B., Venable, K. B., \& Yorke-Smith, N. (2007). Strong controllability of disjunctive temporal problems with uncertainty. In Proceedings of the 13th International Conference on Principles and Practice of Constraint Programming (CP-2007), pp. $856-863$.

Rossi, F., Sperduti, A., Venable, K. B., Khatib, L., Morris, P. H., \& Morris, R. A. (2002). Learning and solving soft temporal constraints: An experimental study. In Proceed- 
ings of the 8th International Conference on Principles and Practice of Constraint Programming, pp. 249-263.

Rossi, F., Venable, K. B., \& Yorke-Smith, N. (2006). Uncertainty in soft temporal constraint problems: A general framework and controllability algorithms for the fuzzy case. Journal of Artificial Intelligence Research, 27, 617-674.

Stergiou, K., \& Koubarakis, M. (1998a). Backtracking algorithms for disjunctions of temporal constraints. In Proceedings of the 15th National Conference on Artificial Intelligence (AAAI-1998), pp. 248-253.

Stergiou, K., \& Koubarakis, M. (1998b). Backtracking algorithms for disjunctions of temporal constraints. Artificial Intelligence, 120, 248-253.

Tsamardinos, I. (2002). A probabilistic approach to robust execution of temporal plans with uncertainty. In Methods and Applications of Artificial Intelligence: Proceedings of the Second Hellenic Conference on Artificial Intelligence, Vol. 2308 of Lecture Notes in Computer Science, pp. 97-108.

Tsamardinos, I., Vidal, T., \& Pollack, M. (2003). CTP: A new constraint-based formalism for conditional, temporal planning. Constraints, 8, 365-388.

Venable, K. B., \& Yorke-Smith, N. (2005). Disjunctive temporal planning with uncertainty. In Proceedings of the 19th International Joint Conference on Artificial Intelligence (IJCAI-2005), pp. 1721-1722.

Vidal, T., \& Fargier, H. (1999). Handling contingency in temporal constraint networks: from consistency to controllabilities. Journal of Experimental and Theoretical Artificial Intelligence, 11, 23-45.

Wachter, A., \& Biegler, L. T. (2006). On the implementation of an interior-point filter linesearch algorithm for large-scale nonlinear programming. Mathematical Programming, $106,25-57$.

Wah, B. W., \& Xin, D. (2007). Optimization of bounds in temporal flexible planning with dynamic controllability. International Journal on Artificial Intelligence Tools, 16(1), $17-44$.

Williams, B. C., \& Ragno, R. J. (2002). Conflict-directed A* and its role in model-based embedded systems. Journal of Discrete Applied Mathematics, 155(12), 1562-1595.

Yu, P., Fang, C., \& Williams, B. (2014). Resolving uncontrollable conditional temporal problems using continuous relaxations. In Proceedings of the Twenty-fourth International Conference on Automated Planning and Scheduling (ICAPS-2014), pp. 341-349.

Yu, P., Fang, C., \& Williams, B. (2015). Resolving over-constrained probabilistic temporal problems. In Proceedings of the Twenty-Ninth AAAI Conference on Artificial Intelligence (AAAI-2015), pp. 3425-3431.

Yu, P., \& Williams, B. (2013). Continuously relaxing over-constrained conditional temporal problems through generalized conflict learning and resolution. In Proceedings of the 23th International Joint Conference on Artificial Intelligence (IJCAI-2013), pp. 24292436. 